\title{
ON QUATERNIONIC COMPLEXES OVER UNIMODULAR QUATERNIONIC MANIFOLDS
}

\author{
WEI WANG
}

\begin{abstract}
Penrose's two-spinor notation for 4-dimensional Lorentzian manifolds is extended to twocomponent notation for quaternionic manifolds, which is a useful tool for calculation. We can construct a family of quaternionic complexes over unimodular quaternionic manifolds only by elementary calculation. On complex quaternionic manifolds as complexification of quaternionic Kähler manifolds, the existence of these complexes was established by Baston by using twistor transformations and spectral sequences. Unimodular quaternionic manifolds constitute a large nice class of quaternionic manifolds: there exists a very special curvature decomposition; the conformal change of a unimodular quaternionic structure is still unimodular quaternionic; the complexes over such manifolds are conformally invariant. This class of manifolds is the real version of torsion-free QCFs introduced by Bailey and Eastwood. These complexes are elliptic. We also obtain a Weitzenböck formula to establish vanishing of the cohomology groups of these complexes for quaternionic Kähler manifolds with negative scalar curvatures.
\end{abstract}

\section{INTRODUCTION}

Quaternionic manifolds are important in supersymmetric theory in physics, in particular in nonlinear sigma models. It is well known that the supersymmetric sigma models are deeply related to geometries with complex structures: the target manifold must be a Kähler manifold in $N=1$ theories; it must be a hyperKähler manifold in rigid $N=2$ supersymmetric theories; while in local supersymmetric $N=2$ theories, it must be a quaternionic Kähler manifold of negative curvature, etc.. The quaternionic complexes over quaternionic Kähler manifolds were used to investigate $N=2$ supersymmetric black holes recently [16]. The Baston operator in the these complexes appears in quantization of $N=2$ supergravity black holes [27. Physicists are also interested in supersymmetric and superconformal theory over more general quaternionic manifolds $[8$. The geometry of quaternionic manifolds is an active direction of research in last four decades (cf. e.g. 6] [14, 20] 24, 32, 33, 38] 39] and references therein).

Recall that an almost quaternionic structure on a manifold $M$ is a rank-3 subbundle of EndTM which is locally spanned by three almost complex structures on $T M$ satisfying the commutating relation of quaternions, i.e. the frame bundle of $M$ reduces to a principal bundle $P$ with structure group $\mathrm{GL}(n, \mathbb{H}) \mathrm{Sp}(1) \cong \mathrm{GL}(n, \mathbb{H}) \times_{\mathbb{Z}_{2}} \mathrm{Sp}(1)$, where $\mathrm{Sp}(1)$ is the Lie group of right multiplying unit quaternions. It is a quaternionic manifold if there exists a torsion-free connection on $P$. It is called quaternionic Kähler if the Levi-Civita connection for the metric preserves the quaternionic structure, i.e. the frame bundle of $M$ reduces to a principal $\operatorname{Sp}(n) \operatorname{Sp}(1)$-bundle with a torsion-free connection. A quaternionic manifold $M$ is called unimodular if the quaternionic connection preserves a volume form on $M$, i.e. the frame bundle of $M$ reduces to a principal $\mathrm{SL}(n, \mathbb{H}) \mathrm{Sp}(1)$-bundle with a torsion-free connection.

Given a representation $W$ of $\mathrm{GL}(n, \mathbb{H}) \times \operatorname{Sp}(1)$ (a double covering of $\operatorname{GL}(n, \mathbb{H}) \operatorname{Sp}(1)$ ), choose a lift of $P$ to a principal $\mathrm{GL}(n, \mathbb{H}) \times \mathrm{Sp}(1)$-bundle $\widetilde{P}$. Then we can define the associated bundle $\widetilde{P} \times_{\mathrm{GL}(n, \mathbb{H}) \times \operatorname{Sp}(1)} W$.

Supported by National Nature Science Foundation in China (No. 11571305)

Department of Mathematics, Zhejiang University, Zhejiang 310027, P. R. China, Email: wwang@zju.edu.cn. 
Such a lifting always exists locally, and exists globally when the obstruction to the lifting in $H^{2}\left(M, \mathbb{Z}_{2}\right)$ vanishes, e.g. when it is a $8 n$-dimensional quaternionic Kähler manifold, (cf. section 2 in 32 and section 2 in [33). In the sequel, we assume that such a lifting always exists. Taking the standard GL( $n, \mathbb{H})$-module $\mathbb{C}^{2 n}$ and $\operatorname{Sp}(1)$-module $\mathbb{C}^{2}$, we have associated vector bundles

$$
E:=\widetilde{P} \times_{\mathrm{GL}(n, \mathbb{H}) \times \mathrm{Sp}(1)} \mathbb{C}^{2 n}, \quad H:=\widetilde{P} \times_{\mathrm{GL}(n, \mathbb{H}) \times \mathrm{Sp}(1)} \mathbb{C}^{2}, \quad T M \cong \widetilde{P} \times_{\mathrm{GL}(n, \mathbb{H}) \times \mathrm{Sp}(1)} \mathbb{H}^{n} .
$$

A connection on the principal bundle $P$ is trivially lifted to the principal bundle $\widetilde{P}$, and so induces connections on associated vector bundle $E, H$ and $T M$, respectively. It is well known [32] 33] that the complexified tangent bundle of an almost quaternionic manifold $M$ decomposes as the tensor product

$$
\mathbb{C} T M \cong E \otimes H .
$$

Denote by $\Gamma(V)$ the space of smooth sections of a vector bundle $V$ over $M$. In this paper we will discuss the construction of a family of elliptic differential complexes over a unimodular quaternionic manifold $M$ ( $M$ is right conformally flat when $\operatorname{dim}_{\mathbb{R}} M=4$ ):

$$
\begin{aligned}
0 \longrightarrow \Gamma\left(\odot^{k} H^{*}\right) & \stackrel{D_{0}^{(k)}}{\longrightarrow} \Gamma\left(\Lambda^{1} E^{*} \otimes \odot^{k-1} H^{*}\right) \stackrel{D_{1}^{(k)}}{\longrightarrow} \cdots \cdots \longrightarrow \Gamma\left(\Lambda^{k} E^{*}\right) \stackrel{D_{k}^{(k)}}{\longrightarrow} \Gamma\left(\Lambda^{k+2} E^{*}\right) \\
& \stackrel{D_{k+1}^{(k)}}{\longrightarrow} \Gamma\left(\Lambda^{k+3} E^{*} \otimes H\right) \longrightarrow \cdots \cdots \stackrel{D_{2 n-2}^{(k)}}{\longrightarrow} \Gamma\left(\Lambda^{2 n} E^{*} \otimes \odot^{2 n-k-2} H\right) \longrightarrow 0,
\end{aligned}
$$

$k=0,1, \ldots$, where $\Lambda^{q} E^{*}$ is the $q$-th exterior product of $E^{*}$, and $\odot^{p} H^{*}$ is the $p$-th symmetric product of $H^{*}$. The first operator $D_{0}^{(k)}$ is called the $k$-Cauchy-Fueter operator. $D_{j}^{(k)}$ 's are differential operators of the first order except $D_{k}^{(k)}$, which is of the second order (cf. Theorem 3.1). By using the twistor transformation and spectral sequences, Baston [6] proved the existence of these complexes over complex quaternionic manifolds. He generalized the result and the complex geometric method of Eastwood, Penrose and Wells 19 for $n=1$. A complex quaternionic manifold $\widetilde{M}$ with $\operatorname{dim}_{\mathbb{C}} \widetilde{M}=4 n$ is a complex manifold whose holomorphic tangential bundle decompose as $T \widetilde{M} \cong \widetilde{E} \otimes \widetilde{H}$, where $\widetilde{E}$ and $\widetilde{H}$ are holomorphic vector bundles of dimension $2 n$ and 2, respectively, and there exists a torsion-free holomorphic connection preserving symplectic forms $\epsilon$ on $\widetilde{E}$ and $\varepsilon$ on $\widetilde{H}$. Then $\epsilon \otimes \varepsilon$ is a complex Riemannian metric on $\widetilde{M}$. Baston used Levi-Civita connection on $\widetilde{M}$ to construct these complexes after section 2.6 in his paper [6]. So he constructed complexes over quaternionic Kähler manifolds. Because the twistor transformation is a complicated technique in complex geometry, it is interesting to construct complexes (1.3) by only using elementary method of differential geometry.

Another motivation to consider these complexes comes from the function theory of several quaternionic variables. We write a vector in the quaternionic space $\mathbb{H}^{n}$ as $\mathbf{q}=\left(\mathbf{q}_{0}, \ldots, \mathbf{q}_{n-1}\right)$ with $\mathbf{q}_{l}=x_{4 l+1}+x_{4 l+2} \mathbf{i}+$ $x_{4 l+3} \mathbf{j}+x_{4 l+4} \mathbf{k} \in \mathbb{H}, l=0,1, \ldots, n-1$. The usual Cauchy-Fueter operator is defined as

$$
\mathscr{D}: C^{1}\left(\mathbb{H}^{n}, \mathbb{H}\right) \rightarrow C\left(\mathbb{H}^{n}, \mathbb{H}^{n}\right), \quad \mathscr{D} f=\left(\begin{array}{c}
\bar{\partial}_{\mathbf{q}_{0}} f \\
\vdots \\
\bar{\partial}_{\mathbf{q}_{n-1}} f
\end{array}\right)
$$

for $f \in C^{1}\left(\mathbb{H}^{n}, \mathbb{H}\right)$, where $\bar{\partial}_{\mathbf{q}_{l}}=\partial_{x_{4 l+1}}+\mathbf{i} \partial_{x_{4 l+2}}+\mathbf{j} \partial_{x_{4 l+3}}+\mathbf{k} \partial_{x_{4 l+4}}, l=0,1, \ldots, n-1$. A function $f: \mathbb{H}^{n} \rightarrow \mathbb{H}$ is called (left) regular if $\mathscr{D} f \equiv 0$ on $\mathbb{H}^{n}$. As in the theory of several complex variables, to investigate regular quaternionic functions, it is important to solve the non-homogeneous Cauchy-Fueter equation

$$
\mathscr{D} f=h
$$


for prescribed $h \in C^{2}\left(\Omega, \mathbb{H}^{n}\right)$ over a domain $\Omega$. This system is overdetermined, i.e. the number of equations is larger than the number of unknown functions for $n>1$. So for (1.4) to be solvable, $h$ must satisfy some compatible condition. This condition was found by Adams, Loustaunau, Palamodov and Struppa 1] with the help of computer algebra method, namely,

$$
\mathscr{D}_{1} h=0
$$

for some differential operator of second order $\mathscr{D}_{1}: C^{2}\left(\Omega, \mathbb{H}^{n}\right) \rightarrow C\left(\Omega, \Lambda^{3} \mathbb{H}^{n}\right)$. In fact, there exists a differential complex corresponding to the Dolbeault complex in the theory of several complex variables:

$$
0 \rightarrow \Gamma(\Omega, \mathbb{H}) \stackrel{\mathscr{D}}{\longrightarrow} \Gamma\left(\Omega, \mathbb{H}^{n}\right) \stackrel{\mathscr{D}_{1}}{\longrightarrow} \Gamma\left(\Omega, \Lambda^{3} \mathbb{H}^{n}\right) \rightarrow \cdots \cdots \rightarrow 0,
$$

called the Cauchy-Fueter complex (cf. [17] [18] and references therein). It was realized later that the Cauchy-Fueter operator is exactly the 1-Cauchy-Fueter operator and the Cauchy-Fueter complex 10. 17. is equivalent to a sequence obtained by Baston in [6], although Baston's result is a complexified version. In [45, the author introduced notions of the $k$-Cauchy-Fueter operator on the quaternionic space $\mathbb{H}^{n}$ and $k$-regular functions annihilated by this operator. In the function theory, (1.3) is called the $k$-Cauchy-Fueter complex. The $k$-Cauchy-Fueter complex over $\mathbb{H}^{n}$ was explicitly written down by using the twistor transformation and spectral sequences [45] (see also [9]). By solving the non-homogeneous $k$-Cauchy-Fueter equations, we showed the Hartogs' phenomenon for $k$-regular functions [45]. To develop the function theory over curved manifolds, we need to write down these complexes on manifolds explicitly.

The $k$-Cauchy-Fueter operator over the 1-dimensional quaternionic space $\mathbb{H}$ also has the origin in physics: it is the elliptic version of spin $k / 2$ massless field operator [15] [19] [30] [31] over the Minkowski space: $D_{0}^{(1)} \phi=0$ corresponds to the Dirac-Weyl equation whose solutions correspond to neutrinos; $D_{0}^{(2)} \phi=0$ corresponds to the Maxwell equation whose solutions correspond to photons; $D_{0}^{(3)} \phi=0$ corresponds to the Rarita-Schwinger equation; $D_{0}^{(4)} \phi=0$ corresponds to linearized Einstein's equation whose solutions correspond to weak gravitational fields, etc..

Salamon [33] constructed another family of quaternionic complexes over quaternionic manifolds:

$$
\begin{aligned}
0 \longrightarrow \Gamma\left(\odot^{k} H^{*}\right) & \longrightarrow \Gamma\left(\Lambda^{1} E^{*} \otimes \odot^{k+1} H^{*}\right) \longrightarrow \cdots \cdots \longrightarrow \Gamma\left(\Lambda^{p} E^{*} \otimes \odot^{k+p} H^{*}\right) \\
& \longrightarrow \Gamma\left(\Lambda^{p+1} E^{*} \otimes \odot^{k+p+1} H^{*}\right) \longrightarrow \cdots \cdots .
\end{aligned}
$$

The half sequence of the $k$-Cauchy-Fueter complex (1.3), beginning with the operator $D_{k+1}^{(k)}$, is similar to Salamon's complexes. In last two decades, quaternionic manifolds were also studied from the point of view of parabolic geometry (cf. [11] [12] [14 [36] [17, and reference therein). Several interesting differential complexes over curved quaternionic manifolds have been constructed from BGG-sequences [13] 14. Recall that for a parabolic subalgebra $\mathfrak{p}$ (resp. subgroup $P$ ) of a complex semisimple Lie algebra $\mathfrak{g}$ (resp. group $G$ ), let $E(\lambda)$ be the irreducible $\mathfrak{p}$-module with the lowest weight $-\lambda$. Denote by $\mathscr{O}_{\mathfrak{p}}(\lambda)$ the sheaf of holomorphic sections of vector bundle associated to $E(\lambda)$ over $G / P$. A general $B G G$-sequence is an exact sequence

$$
0 \longrightarrow E_{\mathfrak{g}}(\lambda) \longrightarrow \mathscr{O}_{\mathfrak{p}}(\lambda) \stackrel{d_{0}}{\longrightarrow} \bigoplus_{w \in W^{\mathfrak{p}}, l(w)=1} \mathscr{O}_{\mathfrak{p}}(w \cdot \lambda) \stackrel{d_{1}}{\longrightarrow} \bigoplus_{w \in W^{\mathfrak{p}}, l(w)=2} \mathscr{O}_{\mathfrak{p}}(w \cdot \lambda) \longrightarrow \cdots,
$$

for a dominant weight $\lambda$ of $\mathfrak{g}$, where $W^{\mathfrak{p}}$ is the Hasse diagram associated to $\mathfrak{p}$ (cf. theorem 8.4.1 in [7]). $E_{\mathfrak{g}}(\lambda)$ is a finite dimensional irreducible representation of $\mathfrak{g}$. But on the flat space $\mathbb{H}^{n}$, the $k$-CauchyFueter complex after complexification is a sequence (1.7) with the weight $\lambda$ singular for $\mathfrak{g}$, but dominant for $\mathfrak{p}$ (cf. theorem 11 in [6]). In this case, $E_{\mathfrak{g}}(\lambda)$ is an infinite dimensional irreducible representation 
of $\mathfrak{g}$. So it is not a BGG-sequence. Moreover, $D_{k}^{(k)}$ is an example of non-standard invariant operators (cf. Remark 12 in 17). In general, it is not easy to construct an exact sequence with singular weights. People usually construct such a sequence from a relative BGG sequence, case by case, by using the twistor method (cf. e.g. [7 [29] [46] and references therein) or the method of cohomology parabolic induction in the representation theory (cf. section 11.3 of [7]). From the point of view of function theory, we are especially interested in differential complexes (1.7) with singular weights, because only in this case "regular functions" as elements of ker $d_{0} \cong E_{\mathfrak{g}}(\lambda)$ are abundant. On the flat space $\mathbb{H}^{n}$, a generalized Penrose integral formula provides all solutions to the $k$-Cauchy-Fueter equation, which is of infinite dimensional (cf. 25]).

The 0-Cauchy-Fueter operator $D_{0}^{(0)}: \Gamma(M, \mathbb{C}) \rightarrow \Gamma\left(M, \Lambda^{2} E^{*}\right)$ is called the Baston operator. Certain exterior product of this operator gives us the quaternionic Monge-Ampère operator by Alesker [2]. This interpretation together with the second operator $D_{1}^{(0)}$ allows us to develop pluripotential theory over $\mathbb{H}^{n}$ [40] [41] [42] 43]. To develop pluripotential theory over curved quaternionic manifolds, in particular to study the quaternionic Calabi-Yau problem on quaternionic manifolds [3] 4, we need to know 0-CauchyFueter complex on manifolds explicitly.

Penrose's two-spinor notation is useful for studying 4-dimensional manifolds [30] 31. It is generalized to complex quaternionic manifolds by Baston [6] and to more general complex paraconformal manifolds by Bailey and Eastwood [5. As a real version, we extend this notation to quaternionic manifolds simply by realizing the isomorphism $\mathbb{C} T M \cong E \otimes H$ in (1.2): for local frames $\left\{e_{A}\right\}$ and $\left\{e_{A^{\prime}}\right\}$ of $E$ and $H$, respectively, we identify $e_{A} \otimes e_{A^{\prime}}$ with a complex tangential vector $Z_{A A^{\prime}}$ (see section 2.1). The quaternionic connection on $M$ induces a $\mathfrak{g l}(2 n, \mathbb{C})$-connection on $E$ and a $\mathfrak{s u}(2)$-connection on $H$, respectively, and so the curvature of the quaternionic connection has two components

$$
R_{a b A}{ }^{B} \quad \text { and } \quad R_{a b A^{\prime}}{ }^{B^{\prime}},
$$

corresponding to curvatures of the bundles $E$ and $H$, respectively. Here we use indices $A, B$ and $A^{\prime}, B^{\prime}$ for components of sections of bundles $E$ and $H$, respectively, and indices $a, b$ for components of the local quaternionic frame of the tangent bundle $T M$. Furthermore, curvatures of a unimodular quaternionic connection have a very special decomposition (cf. Proposition 2.2), with the help of which we can check that the sequence (1.3) is a complex, i.e. $D_{j+1}^{(k)} \circ D_{j}^{(k)}=0$, by direct calculation in Section 3.1. Twocomponent notation is a useful tool for calculation over a quaternionic manifold and everything in this paper is based on elementary calculation by this notation. Unimodular quaternionic manifolds constitute a nice class of quaternionic manifolds, because the conformal change of a unimodular quaternionic structure is still unimodular quaternionic, while the conformal change of a quaternionic Kähler structure is usually not quaternionic Kähler (cf. [28). We also give the conformal transformation formula of these operators $D_{j}^{(k)}$ in Section 3.1.

In Section 3.2, we show that the $k$-Cauchy-Fueter complex is elliptic, i.e. its symbol complex is a exact sequence of complex vector spaces. Write the $k$-Cauchy-Fueter complex as

$$
0 \longrightarrow \Gamma\left(\mathscr{V}_{0}^{(k)}\right) \stackrel{D_{0}^{(k)}}{\longrightarrow} \Gamma\left(\mathscr{V}_{1}^{(k)}\right) \stackrel{D_{1}^{(k)}}{\longrightarrow} \cdots \longrightarrow \Gamma\left(\mathscr{V}_{2 n-1}^{(k)}\right) \longrightarrow 0,
$$

where $\mathscr{V}_{j}^{(k)}$ is the $j$-th vector space in the sequence (1.3). By the theory of elliptic operators, we know the Hodge-type decomposition and that the $j$-th cohomology group

$$
H_{(k)}^{j}(M)=\operatorname{ker} D_{j}^{(k)} / \operatorname{Im} D_{j-1}^{(k)}
$$


of the $k$-Cauchy-Fueter complex over a compact unimodular quaternionic manifold (right conformally flat if $\operatorname{dim}_{\mathbb{R}} M=4$ ) is finite dimensional, and can be represented by Hodge-type elements.

In Section 4, we prove a Weitzenböck formula for these complexes over a quaternionic Kähler manifold $M$, and show a vanishing theorem for the cohomologies $H_{(k)}^{j}(M), j=1, \ldots, k-1$, if its scalar curvature is negative. The Weizenböck formula and vanishing theorem for Salamon's complexes over such manifolds with negative scalar curvatures were already given by Horan [22] (see also Homma 21] and NagatomoNitta [26]). The latter one essentially gives us the result for the $k$-Cauchy-Fueter complex for $j \geq k+3$.

I would like to thank the referee for many valuable suggestions.

\section{UNIMODULAR QUATERNIONIC MANIFOLDS AND THEIR CURVATURES}

2.1. Realization of the isomorphism $\mathbb{C} T M \cong E \otimes H$. Denote by $\operatorname{GL}(n, \mathbb{H})$ the group of all invertible quaternionic $(n \times n)$-matrices. $\operatorname{Sp}(n):=\left\{A \in \mathrm{GL}(n, \mathbb{H}) ; \bar{A}^{t} A=A^{t}=I_{n \times n}\right\} . \quad \mathfrak{s l}(n, \mathbb{H}):=\{A \in$ $\mathfrak{g l}(n, \mathbb{H}) ; \operatorname{Re}(\operatorname{tr}(A))=0\}$. We denote by $\operatorname{SL}(n, \mathbb{H})$ the connected component containing the identity of the Lie group with Lie algebra $\mathfrak{s l}(n, \mathbb{H})$.

Let $A=\left(A_{j k}\right)_{p \times m}$ be a quaternionic $(l \times m)$-matrix and write $A_{j k}=a_{j k}^{1}+\mathbf{i} a_{j k}^{2}+\mathbf{j} a_{j k}^{3}+\mathbf{k} a_{j k}^{4} \in \mathbb{H}$. We define $\tau(A)$ to be the complex $(2 p \times 2 m)$-matrix

$$
\tau(A)=\left(\begin{array}{cccc}
\tau\left(A_{00}\right) & \tau\left(A_{01}\right) & \ldots & \tau\left(A_{0(m-1)}\right) \\
\tau\left(A_{10}\right) & \tau\left(A_{11}\right) & \ldots & \tau\left(A_{1(m-1)}\right) \\
\cdots & \cdots & \cdots & \cdots \\
\tau\left(A_{(p-1) 0}\right) & \tau\left(A_{(p-1) 1}\right) & \cdots & \tau\left(A_{(p-1)(m-1)}\right)
\end{array}\right),
$$

where $\tau\left(A_{j k}\right)$ is the complex $(2 \times 2)$-matrix

$$
\left(\begin{array}{cr}
a_{j k}^{1}+\mathbf{i} a_{j k}^{2} & -a_{j k}^{3}-\mathbf{i} a_{j k}^{4} \\
a_{j k}^{3}-\mathbf{i} a_{j k}^{4} & a_{j k}^{1}-\mathbf{i} a_{j k}^{2}
\end{array}\right) .
$$

This is motivated by the embedding of quaternionic numbers into $2 \times 2$-matrices. The definition of $\tau$ above and the following proposition are the conjugate version of those in [43].

Proposition 2.1. (proposition 2.1 in [43]) (1) $\tau(A B)=\tau(A) \tau(B)$ for a quaternionic $(p \times m)$-matrix $A$ and a quaternionic $(m \times l)$-matrix $B$. In particular, for $q^{\prime}=A q, q, q^{\prime} \in \mathbb{H}^{n}, A \in G L(n, \mathbb{H})$, we have

$$
\tau\left(q^{\prime}\right)=\tau(A) \tau(q)
$$

as complex $(2 n \times 2)$-matrices.

(2) For $A \in G L(n, \mathbb{H})$, we have

$$
J \overline{\tau(A)}=\tau(A) J, \quad \text { where } \quad J=\left(\begin{array}{ccccc}
0 & 1 & & & \\
-1 & 0 & & & \\
& & 0 & 1 & \\
& & -1 & 0 & \\
& & & & \ddots
\end{array}\right) .
$$

(3) $\tau\left(\bar{A}^{t}\right)=\overline{\tau(A)}^{t}$ for a quaternionic $(n \times n)$-matrix A. If $A \in \operatorname{Sp}(n), \tau(A)$ is symplectic, i.e., $\tau(A) J \tau(A)^{t}=J$. 
Proposition 2.1 (3) implies $\tau(\operatorname{Sp}(n)) \subset \mathrm{SU}(2 n)$. (2.1)-(2.2) implies $\tau(\mathfrak{s l}(n, \mathbb{H})) \subset \mathfrak{s l}(2 n, \mathbb{C})$, and so $\tau(\mathrm{SL}(n, \mathbb{H})) \subset \mathrm{SL}(2 n, \mathbb{C})$. Given the standard volume form on $\mathbb{R}^{4 n}, \mathrm{SL}(n, \mathbb{H})$ is the group consisting of elements of $\mathrm{GL}(n, \mathbb{H})$ which induce transformations of $\mathbb{R}^{4 n}$ preserving this volume form. Let $I_{1}, I_{2}, I_{3}$ be the induced action of $\mathbf{i}, \mathbf{j}, \mathbf{k}$ on the frame bundle. Then we can choose a frame of the tangent bundle

$$
\left(X_{1}, X_{1} I_{1}, X_{1} I_{2}, X_{1} I_{3}, \ldots, X_{4 l+1}, X_{4 l+1} I_{1}, X_{4 l+1} I_{2}, X_{4 l+1} I_{3}, \ldots\right)
$$

called a local quaternionic frame. Label this frame as $\left(X_{1}, \ldots, X_{4 n}\right)$.

$H \otimes E$ is isomorphic to the tangent bundle $\mathbb{C} T M$ as follows. It follows from Proposition 2.1 that $\mathbb{C}^{2 n}$ is a $\operatorname{GL}(n, \mathbb{H})$-module with $A \in \mathrm{GL}(n, \mathbb{H})$ acting on $\mathbb{C}^{2 n}$ by $\tau(A)$, and $\mathbb{C}^{2}$ is a $\mathrm{Sp}(1)$-module with $q \in \operatorname{Sp}(1)$ acting on $\left(z_{1}, z_{2}\right) \in \mathbb{C}^{2}$ by right multiplying the $2 \times 2$-matrix $\tau(q)$. Let $\left\{v_{A}\right\}_{A=0}^{2 n-1},\left\{v_{A^{\prime}}\right\}_{A^{\prime}=0^{\prime}, 1^{\prime}}$ and $\left\{w_{a}\right\}_{a=1}^{4 n}$ be the standard bases of $\mathbb{C}^{2 n}, \mathbb{C}^{2}$ and $\mathbb{R}^{4 n}$, respectively. Write $v_{A A^{\prime}}:=v_{A} \otimes v_{A^{\prime}}$ in $\mathbb{C}^{2 n} \otimes \mathbb{C}^{2}$. The map $\tau$ provides an isomorphism from $\mathbb{C} \otimes \mathbb{H}^{n}$ to $\mathbb{C}^{2 n} \otimes \mathbb{C}^{2}$ as $\mathrm{GL}(n, \mathbb{H}) \times \operatorname{Sp}(1)$-module. Under this identification of $\tau$, we have

$$
\begin{array}{ll}
2 w_{4 l+1}=v_{(2 l) 0^{\prime}}+v_{(2 l+1) 1^{\prime}}, & 2 w_{4 l+2}=-\mathbf{i} v_{(2 l) 0^{\prime}}+\mathbf{i} v_{(2 l+1) 1^{\prime}}, \\
2 w_{4 l+3}=-v_{(2 l) 1^{\prime}}+v_{(2 l+1) 0^{\prime}}, & 2 w_{4 l+4}=\mathbf{i} v_{(2 l) 1^{\prime}}+\mathbf{i} v_{(2 l+1) 0^{\prime}},
\end{array}
$$

by using definition (2.1)-(2.2) of $\tau$ for $m=1$. Thus

$$
\left(\begin{array}{ll}
v_{(2 l) 0^{\prime}} & v_{(2 l) 1^{\prime}} \\
v_{(2 l+1) 0^{\prime}} & v_{(2 l+1) 1^{\prime}}
\end{array}\right)=\left(\begin{array}{rr}
w_{4 l+1}+\mathbf{i} w_{4 l+2} & -w_{4 l+3}-\mathbf{i} w_{4 l+4} \\
w_{4 l+3}-\mathbf{i} w_{4 l+4} & w_{4 l+1}-\mathbf{i} w_{4 l+2}
\end{array}\right) .
$$

Now for a local quaternionic frame $\mathfrak{e}=\left(X_{1}, \ldots, X_{4 n}\right)$, define local sections

$$
e_{A}:=\left(\mathfrak{e}, v_{A}\right), \quad e_{A^{\prime}}:=\left(\mathfrak{e}, v_{A^{\prime}}\right), \quad X_{a}:=\left(\mathfrak{e}, w_{a}\right)
$$

of $E, H$ and $T M$ in (1.1), respectively. Then (2.6) implies that $Z_{A A^{\prime}}=\frac{1}{\sqrt{2}} e_{A} \otimes e_{A^{\prime}}$ are given by

$$
\left(\begin{array}{cc}
Z_{00^{\prime}} & Z_{01^{\prime}} \\
\vdots & \vdots \\
Z_{(2 n-1) 0^{\prime}} & Z_{(2 n-1) 1^{\prime}}
\end{array}\right):=\frac{1}{\sqrt{2}}\left(\begin{array}{cc}
X_{1}+\mathbf{i} X_{2} & -X_{3}-\mathbf{i} X_{4} \\
X_{3}-\mathbf{i} X_{4} & X_{1}-\mathbf{i} X_{2} \\
\vdots & \vdots \\
X_{4 n-3}+\mathbf{i} X_{4 n-2} & -X_{4 n-1}-\mathbf{i} X_{4 n} \\
X_{4 n-1}-\mathbf{i} X_{4 n} & X_{4 n-3}-\mathbf{i} X_{4 n-2}
\end{array}\right),
$$

See (2.20) for the reason to choose factor $\sqrt{2}$ here. This frame over the flat quaternionic space $\mathbb{H}^{n}$ plays an important role in the investigation of quaternionic analysis [25] [40]-44] [45].

Let $\left\{\omega^{i}\right\}$ be the coframe dual to $\left\{X_{j}\right\}$ and let $\left\{e^{A A^{\prime}}\right\}$ be complex 1-forms dual to the two-component local quaternionic frame $\left\{Z_{A A^{\prime}}\right\}$ in (2.8), i.e. $e^{A A^{\prime}}\left(Z_{B B^{\prime}}\right)=\delta_{B}^{A} \delta_{B^{\prime}}^{A^{\prime}}$. It is obvious that

$$
\sqrt{2} e^{00^{\prime}}=\omega^{1}-\mathbf{i} \omega^{2}, \quad \sqrt{2} e^{01^{\prime}}=-\omega^{3}+\mathbf{i} \omega^{4}, \quad \sqrt{2} e^{10^{\prime}}=\omega^{3}+\mathbf{i} \omega^{4}, \quad \sqrt{2} e^{11^{\prime}}=\omega^{1}+\mathbf{i} \omega^{2}, \cdots,
$$

by the expression of $\left\{Z_{A A^{\prime}}\right\}$ in (2.8), and so $\left(e^{00^{\prime}} \wedge e^{11^{\prime}}\right) \wedge\left(e^{10^{\prime}} \wedge e^{01^{\prime}}\right)=-\omega^{1} \wedge \omega^{2} \wedge \omega^{3} \wedge \omega^{4}$. Consequently,

$$
\text { vol }:=(-1)^{n} \omega^{1} \wedge \cdots \wedge \omega^{4 n}=\left(\wedge_{A=0}^{2 n-1} e^{A 0^{\prime}}\right) \wedge\left(\wedge_{B=0}^{2 n-1} e^{B 1^{\prime}}\right) .
$$

A local quaternionic frame $\left\{X_{1}, \ldots, X_{4 n}\right\}$ is called a local unimodular quaternionic frame if the volume form of the manifold is locally given by $\mathrm{vol}$ in (2.9). Note that a local quaternionic frame becomes unimodular simply by multiplying a suitable factor. 
2.2. The two-component notation. Denote by $e^{A^{\prime}}:=\left(\mathfrak{e}, v^{A^{\prime}}\right)$ a local section of the dual bundle $H^{*}$, where $v^{A^{\prime}}$ is the dual of $v_{A^{\prime}}$ in $\mathbb{C}^{2}$. It is similar to define $E^{*}$ and $e^{A}$. Consider

$$
\varepsilon_{A^{\prime} B^{\prime}} e^{A^{\prime}} \otimes e^{B^{\prime}}
$$

where $\varepsilon_{A^{\prime} B^{\prime}}$ is antisymmetric with $\varepsilon_{0^{\prime} 1^{\prime}}=1$. Here and in the following, we use the Einstein's convention of summation over repeated indices. It is a section of the line bundle $\Lambda^{2} H^{*}$ (a symplectic form on $H$ pointwisely). This is because (2.10) is invariant under the action of $\mathrm{Sp}(1)$ by Proposition 2.1 (3). So they can be glued to be a global section. When the manifold is unimodular quaternionic, consider

$$
\epsilon_{A_{1} \ldots A_{2 n}} e^{A_{1}} \otimes \ldots \otimes e^{A_{2 n}}
$$

where $\epsilon_{A_{1} \ldots A_{2 n}}$ is the sign of the permutation from $A_{1}, \ldots, A_{2 n}$ to $1, \ldots, 2 n$. It is a global section of the line bundle $\Lambda^{2 n} E^{*}$, because (2.11) is invariant under the action of $\operatorname{SL}(2 n, \mathbb{C})$.

A section $f$ of $\mathfrak{T}_{q, p}^{l}:=\left(\otimes^{l} H\right) \otimes\left(\otimes^{q} E^{*}\right) \otimes\left(\otimes^{p} H^{*}\right)$ can be written as

$$
f=f_{B_{1} \ldots B_{q} B_{1}^{\prime} \ldots B_{p}^{\prime}}^{A_{1}^{\prime} \ldots A_{1}^{\prime}} e_{A_{1}^{\prime}} \otimes \cdots \otimes e_{A_{l}^{\prime}} \otimes e^{B_{1}} \otimes \cdots \otimes e^{B_{q}} \otimes e^{B_{1}^{\prime}} \otimes \cdots \otimes e^{B_{p}^{\prime}} .
$$

We can identify this section with the tuple of functions

$$
\left(\cdots, f_{B_{1} \ldots B_{q} B_{1}^{\prime} \ldots B_{p}^{\prime}}^{A_{1}^{\prime} \ldots A_{l}^{\prime}}, \cdots\right) \text {. }
$$

A contraction is a map $C: \mathfrak{T}_{q, p+1}^{l+1} \longrightarrow \mathfrak{T}_{q, p}^{l}$ given by $(C f)_{B_{1} \ldots B_{q} B_{1}^{\prime} \ldots B_{p}^{\prime}}^{A_{1}^{\prime} \ldots A^{\prime}}:=f_{B_{1} \ldots B_{q} B_{1}^{\prime} \ldots D^{\prime} \ldots B_{p}^{\prime}}^{A_{1}^{\prime} \ldots D^{\prime}}$, where the superscript and subscript $D^{\prime}$ appear in $j$-th and $\widehat{j}$-th places, respectively. It is a well defined element of $\mathfrak{T}_{q, p}^{l}$ since $e^{D^{\prime}} \otimes e_{D^{\prime}}=\widetilde{e}^{E^{\prime}} \otimes \widetilde{e}_{E^{\prime}}$ under the transformation $\widetilde{e}^{E^{\prime}}=\left(M^{-1}\right)_{D^{\prime}}{ }^{E^{\prime}} e^{D^{\prime}}, \widetilde{e}_{E^{\prime}}=M_{E^{\prime}}{ }^{D^{\prime}} e_{D^{\prime}}$ for $\left(M_{E^{\prime}} D^{\prime}\right) \in \mathrm{Sp}(1)$. We use $\varepsilon_{A^{\prime} B^{\prime}}$ to raise or lower primed indices. For example,

$$
f_{\ldots}{ }^{A^{\prime}} \ldots=f_{\ldots B^{\prime} \ldots} \varepsilon^{B^{\prime} A^{\prime}}, \quad f_{\ldots}{ }^{A^{\prime}} \ldots \varepsilon_{A^{\prime} C^{\prime}}=f_{\ldots C^{\prime} \ldots},
$$

where $\left(\varepsilon^{A^{\prime} B^{\prime}}\right)$ is the inverse of $\left(\varepsilon_{A^{\prime} B^{\prime}}\right)$, i.e., $\varepsilon_{A^{\prime} B^{\prime}} \varepsilon^{B^{\prime} C^{\prime}}=\delta_{A^{\prime}}^{C^{\prime}}=\varepsilon^{C^{\prime} B^{\prime}} \varepsilon_{B^{\prime} A^{\prime}}$. So it is the same after raising and lowering primed indices. $\varepsilon$ has the standard form locally:

$$
\left(\varepsilon_{A^{\prime} B^{\prime}}\right)=\left(\begin{array}{cc}
0 & 1 \\
-1 & 0
\end{array}\right), \quad\left(\varepsilon^{A^{\prime} B^{\prime}}\right)=\left(\begin{array}{cc}
0 & -1 \\
1 & 0
\end{array}\right) .
$$

On a unimodular quaternionic manifold, we can not use $\epsilon$ to raise or lower unprimed indices. This is why we only consider tensors as sections of $\mathfrak{T}_{q, p}^{l}$. But on quaternionic Kähler manifold, we can use $\epsilon_{A B}$ to raise or lower unprimed indices (cf. Section 4).

Recall that a covariant derivative of a vector bundle $V$ is a mapping $\nabla: \Gamma(V) \longrightarrow \Gamma\left((T M)^{*} \otimes V\right)$ satisfying $\nabla(f v)=d f \otimes v+f \nabla v, \nabla\left(v_{1}+v_{2}\right)=\nabla v_{1}+\nabla v_{2}$, for any $v, v_{1}, v_{2} \in \Gamma(V)$ and scalar function $f$. $\nabla$ acts on $V^{*}$ naturally by duality: $X\left(v, v^{*}\right)=\left(\nabla_{X} v, v^{*}\right)+\left(v, \nabla_{X} v^{*}\right)$ for any vector field $X \in T M, v \in \Gamma(V)$ and $v^{*} \in \Gamma\left(V^{*}\right)$. A covariant derivative can be naturally extended to a map $\nabla: \Gamma\left(\left(\otimes^{k} V\right) \otimes\left(\otimes^{l} V^{*}\right)\right) \longrightarrow$ $\Gamma\left((T M)^{*} \otimes\left(\otimes^{k} V\right) \otimes\left(\otimes^{l} V^{*}\right)\right)$.

The quaternionic connection induces an $\mathfrak{g l}(2 n, \mathbb{C})$-connection $\omega^{\prime}$ on $E$ and an $\mathfrak{s u}(2)$-connection $\omega^{\prime \prime}$ on $H$. When the manifold is unimodular or quaternionic Kähler, $\omega^{\prime}$ is $\mathfrak{s l}(2 n, \mathbb{C})$ - or $\mathfrak{s p}(n)$-valued. $\nabla$ is naturally extended to well defined mappings $E \rightarrow(\mathbb{C} T M)^{*} \otimes E$ and $H \rightarrow(\mathbb{C} T M)^{*} \otimes H$ by $\nabla_{X+\mathbf{i} Y}:=\nabla_{X}+\mathbf{i} \nabla_{Y}$, which induce well defined mappings $E^{*} \rightarrow(\mathbb{C} T M)^{*} \otimes E^{*}$ and $H^{*} \rightarrow(\mathbb{C} T M)^{*} \otimes H^{*}$ by duality, and so we get a well defined mapping $\mathfrak{T}_{q, p}^{l} \rightarrow(\mathbb{C} T M)^{*} \otimes \mathfrak{T}_{q, p}^{l}$.

Choose a local quaternionic frame $\mathfrak{e}=\left\{e_{a}:=X_{a}\right\}_{a=1}^{4 n}$ of $T M$ and its dual $\left\{e^{a}\right\}_{a=1}^{4 n}$. Write

$$
\nabla e_{A}=\Gamma_{a A}{ }^{B} e^{a} \otimes e_{B}, \quad \nabla e_{A^{\prime}}=\Gamma_{a A^{\prime}}{ }^{B^{\prime}} e^{a} \otimes e_{B^{\prime}},
$$


where $\Gamma_{a A}{ }^{B}=\omega^{\prime}\left(X_{a}\right)_{A}{ }^{B}$ and $\Gamma_{a A^{\prime}}{ }^{B^{\prime}}=\omega^{\prime \prime}\left(X_{a}\right)_{A^{\prime}}{ }^{B^{\prime}}$ are connection coefficients. Then by duality, we have $\nabla e^{A}=-\Gamma_{a B}{ }^{A} e^{a} \otimes e^{B}, \nabla e^{A^{\prime}}=-\Gamma_{a B^{\prime}}{ }^{A^{\prime}} e^{a} \otimes e^{B^{\prime}}$, which are equivalent to

$$
\nabla_{a} f_{A}=X_{a} f_{A}-\Gamma_{a A}{ }^{D} f_{D}, \quad \nabla_{a} f_{A^{\prime}}=X_{a} f_{A^{\prime}}-\Gamma_{a A^{\prime}}{ }^{D^{\prime}} f_{D^{\prime}} .
$$

In general, $\nabla f$ for $f$ given by (2.12)-(2.13) is the tuple

$$
\left(\cdots, \nabla_{a} f_{B_{1} \ldots B_{q} B_{1}^{\prime} \ldots B_{p}^{\prime}}^{\left.A_{1}^{\prime} \ldots\right)}, \cdots\right)
$$

as a section of $(\mathbb{C} T M)^{*} \otimes \mathfrak{T}_{q, p}^{l} \cong \mathfrak{T}_{q+1, p+1}^{l}$, by the identification (1.2), where

$$
\begin{aligned}
\nabla_{a} f_{B_{1} \ldots B_{q} B_{1}^{\prime} \ldots B_{p}^{\prime}}^{A_{1}^{\prime} \ldots A_{p}^{\prime}}:=X_{a} f_{B_{1} \ldots B_{q} B_{1}^{\prime} \ldots B_{p}^{\prime}}^{A_{1}^{\prime} \ldots A_{1}^{\prime}} & +\Gamma_{a D^{\prime}}^{A_{j}^{\prime}} f_{B_{1} \ldots D^{\prime} \ldots B_{q} B_{1}^{\prime} \ldots B_{p}^{\prime}}^{\prime} \\
& -\Gamma_{a B_{j}}{ }^{D} f_{\ldots D \ldots B_{1}^{\prime} \ldots B_{p}^{\prime}}^{A_{1}^{\prime} \ldots A_{l}^{\prime}}-\Gamma_{a B_{j}^{\prime}}{ }^{D^{\prime}} f_{B_{1} \ldots B_{q} \ldots D^{\prime} \ldots}^{A_{1}^{\prime} \ldots A_{l}^{\prime}}
\end{aligned}
$$

The covariant derivative is invariant after contraction: $\nabla(C f)=C(\nabla f)$, because by (2.15), we have

$$
\left[C\left(\nabla_{a} f\right)-\nabla_{a}(C f)\right]_{B_{1} \ldots B_{q} B_{1}^{\prime} \ldots B_{p}^{\prime}}^{A_{1}^{\prime} \ldots A^{\prime}}=\Gamma_{a E^{\prime}}^{D^{\prime}} f_{B_{1} \ldots B_{q} B_{1}^{\prime} \ldots D^{\prime} \ldots B_{p}^{\prime}}^{A^{\prime} \ldots E^{\prime}}-\Gamma_{a D^{\prime}} E^{\prime} f_{B_{1} \ldots B_{q} B_{1}^{\prime} \ldots E^{\prime} \ldots B_{p}^{\prime}}^{A^{\prime} \ldots D^{\prime}}=0 .
$$

We will use the notation

$$
\nabla_{A A^{\prime}}:=\nabla_{Z_{A A^{\prime}}}=\frac{1}{\sqrt{2}}\left(\nabla_{X_{a}}+\mathbf{i} \nabla_{X_{b}}\right)
$$

if we write $Z_{A A^{\prime}}=\frac{1}{\sqrt{2}}\left(X_{a}+\mathbf{i} X_{b}\right)$ for some $a, b$ (cf. (2.8) $)$. We also write $\nabla_{A A^{\prime}}$ as $\nabla_{A^{\prime} A}$ when it is more convenient. Then

$$
\nabla_{A A^{\prime}} e_{B}=\Gamma_{A A^{\prime} B}{ }^{C} e_{C}, \quad \nabla_{A A^{\prime}} e_{B^{\prime}}=\Gamma_{A A^{\prime} B^{\prime}}{ }^{C^{\prime}} e_{C^{\prime}},
$$

where $\Gamma_{A A^{\prime} B}{ }^{C}=\frac{1}{\sqrt{2}}\left(\Gamma_{a B}{ }^{C}+\mathbf{i} \Gamma_{b B}{ }^{C}\right)$ and $\Gamma_{A A^{\prime} B^{\prime}}{ }^{C^{\prime}}=\frac{1}{\sqrt{2}}\left(\Gamma_{a B^{\prime}} C^{\prime}+\mathbf{i} \Gamma_{b B^{\prime}} C^{\prime}\right)$. The formula (2.15) holds for $a=A A^{\prime}$. Denote

$$
2 \nabla_{[a} \nabla_{b]}:=\nabla_{a} \nabla_{b}-\nabla_{b} \nabla_{a} .
$$

The torsion is defined as $2 \nabla_{[a} \nabla_{b]} \phi=T_{a b}{ }^{c} \nabla_{c} \phi$ for any scalar function $\phi$. Then by definition $T_{a b}{ }^{c}=$ $\Gamma_{a b}{ }^{c}-\Gamma_{b a}{ }^{c}+C_{a b}{ }^{c}$ where the numbers $C_{a b}{ }^{c}$ are given by $\left[X_{a}, X_{b}\right]=C_{a b}{ }^{c} X_{c}$. It is direct to check that

$$
\left(\nabla_{a} \nabla_{b}-\nabla_{b} \nabla_{a}\right)\left(\phi f_{A}\right)=T_{a b}{ }^{c} \nabla_{c} \phi f_{A}+\phi\left(\nabla_{a} \nabla_{b}-\nabla_{b} \nabla_{a}\right) f_{A}
$$

for a scalar function $\phi$, by the formula (2.15) for covariant derivatives. So when the connection is torsionfree, $2 \nabla_{[a} \nabla_{b]}$ is an endomorphism of $\Gamma\left(E^{*}\right)$ as a $C^{\infty}(M)$-module for fixed $a, b$ (similarly for $\Gamma\left(H^{*}\right)$ ). So we can write

$$
2 \nabla_{[a} \nabla_{b]} f_{A}:=-R_{a b A}^{D} f_{D}, \quad 2 \nabla_{[a} \nabla_{b]} f_{A^{\prime}}:=-R_{a b A^{\prime}} D^{\prime} f_{D^{\prime}}
$$

By (2.15), we see that

$$
\nabla_{[a} \nabla_{b]}\left(f_{\mathscr{A}} h_{\mathscr{B}}\right)=\nabla_{[a} \nabla_{b]} f_{\mathscr{A}} \cdot h_{\mathscr{B}}+f_{\mathscr{A}} \cdot \nabla_{[a} \nabla_{b]} h_{\mathscr{B}} .
$$

In general, we have the generalized Ricci identity:

$$
2 \nabla_{[a} \nabla_{b]} f_{B_{1} \ldots B_{q} B_{1}^{\prime} \ldots B_{p}^{\prime}}^{A_{1}^{\prime} \ldots A^{\prime}}:=R_{a b D^{\prime}}^{A_{j}^{\prime}} f_{B_{1} \ldots B^{\prime} \ldots B_{q} B_{1}^{\prime} \ldots B_{p}^{\prime}}-R_{a b B_{j}}{ }^{D} f_{\ldots D \ldots B_{1}^{\prime} \ldots B_{p}^{\prime}}^{A_{1}^{\prime} \ldots A^{\prime}}-R_{a b B_{j}^{\prime}}^{D^{\prime}} f_{B_{1} \ldots B_{q} \ldots D^{\prime} \ldots}^{A_{1}^{\prime} \ldots A_{1}^{\prime}}
$$

See Penrose-Rindler [30] 31] or Bailey-Eastwood [5].

If the manifold is unimodular quaternionic, the connection on $E$ preserves the $2 n$-form $\epsilon$ (2.11), i.e.

$$
\nabla_{a} \epsilon_{A_{1} \ldots A_{2 n}}=\Gamma_{a A_{j}}{ }^{D} \epsilon_{A_{1} \ldots D \ldots A_{2 n}}=\Gamma_{a D}{ }^{D} \epsilon_{A_{1} \ldots A_{2 n}}=0
$$


where $\epsilon_{A_{1} \ldots A_{2 n}}$ is nonzero only if $\left(A_{1} \ldots A_{2 n}\right)$ is a permutation of $(0, \ldots, 2 n-1)$ and $\left(\Gamma_{a A}{ }^{D}\right)$ is $\mathfrak{s l}(2 n, \mathbb{C})$ valued. Similarly, the symplectic form $\varepsilon_{A^{\prime} B^{\prime}}$ on $H$ is also preserved by the connection on $H$, i.e.

$$
\nabla_{a} \varepsilon_{A^{\prime} B^{\prime}}=0 .
$$

Thus when the manifold is quaternionic Kähler, $\nabla$ is a connection of $\mathbb{C} T M=H \otimes E$ compatible with metric

$$
g_{a b}=g\left(Z_{A A^{\prime}}, Z_{B B^{\prime}}\right)=\varepsilon_{A B} \varepsilon_{A^{\prime} B^{\prime}}, \quad a=A A^{\prime}, \quad b=B B^{\prime},
$$

for $Z_{A A^{\prime}}$ in (2.8) if we choose local quaternionic frame $\left\{X_{a}\right\}$ in (2.8) orthonormal.

The notion of unimodular quaternionic structure is a real version of the notion of torsion-free QCFstructure on a complex manifold introduced by Bailey and Eastwood [5]. A quaternionic conformal structure (briefly $Q C F$ ) on a $4 n$-dimensional complex quaternionic manifold $\widetilde{M}$ is given by an isomorphism between $T \widetilde{M}$ and $\widetilde{E} \otimes \widetilde{H}$ and a fixed isomorphism between $\Lambda^{2 n} \widetilde{E}^{*}$ and $\Lambda^{2} \widetilde{H}^{*}$. Given a symplectic form $\widetilde{\varepsilon}$ in $\Lambda^{2} \widetilde{H}^{*}$, the isomorphism induces a $2 n$-form $\widetilde{\epsilon}$ in $\Lambda^{2 n} \widetilde{E}^{*}$, and there exists a unique connection $\nabla$ preserving $\widetilde{\epsilon}$ and $\widetilde{\varepsilon}$ (cf. theorem 2.4 in Bailey-Eastwood [5]). The QCF-structure is called torsion-free if the induced connection on the holomorphic tangent bundle is torsion-free.

The curvature of the complexified tangent bundle is

$$
R_{A A^{\prime} B B^{\prime} C C^{\prime}}{ }^{D D^{\prime}} f^{C C^{\prime}}=\left(\nabla_{A A^{\prime}} \nabla_{B B^{\prime}}-\nabla_{B B^{\prime}} \nabla_{A A^{\prime}}\right) f^{D D^{\prime}}
$$

if we identify $\left(f^{D D^{\prime}}\right)$ with a local section of $\mathbb{C} T M$. By the generalized Ricci identity, we see that the curvature has the decomposition:

$$
R_{A A^{\prime} B B^{\prime} C C^{\prime}}{ }^{D D^{\prime}}=R_{A A^{\prime} B B^{\prime} C}{ }^{D} \delta_{C^{\prime}}^{D^{\prime}}+R_{A A^{\prime} B B^{\prime} C^{\prime}}^{D^{\prime}} \delta_{C}{ }^{D} .
$$

We will use the following notations: for $a=A A^{\prime}, b=B B^{\prime}$,

$$
R_{A^{\prime} B^{\prime} A B C}{ }^{D}:=R_{A A^{\prime} B B^{\prime} C^{D}}=R_{a b C}{ }^{D}, \quad R_{A B A^{\prime} B^{\prime} C^{\prime}}{ }^{D^{\prime}}:=R_{A A^{\prime} B B^{\prime} C^{\prime}}{ }^{D^{\prime}}=R_{a b C^{\prime}}{ }^{D^{\prime}} .
$$

Corollary 2.1. On a unimodular quaternionic manifold, we have

$$
R_{A^{\prime} B^{\prime} A B C}{ }^{C}=0, \quad R_{A B A^{\prime} B^{\prime} C^{\prime}} C^{\prime}=0 .
$$

Proof. Note that $\left(\nabla_{A A^{\prime}} \nabla_{B B^{\prime}}-\nabla_{B B^{\prime}} \nabla_{A A^{\prime}}\right) \epsilon_{A_{1} \ldots A_{2 n}}=0$ by (2.18), which implies that

$$
R_{A^{\prime} B^{\prime} A B A_{j}}{ }^{D} \epsilon_{A_{1} \ldots D \ldots A_{2 n}}=0
$$

by the generalized Ricci identity again. Noting that $\epsilon_{A_{1}, \ldots, A_{2 n}}$ is nonzero only if $\left(A_{1} \ldots A_{2 n}\right)$ is a permutation of $(0, \ldots, 2 n-1)$, we find the first trace vanishing. It is similar for the second one.

We will use symmetrisation and antisymmetrisation of indices

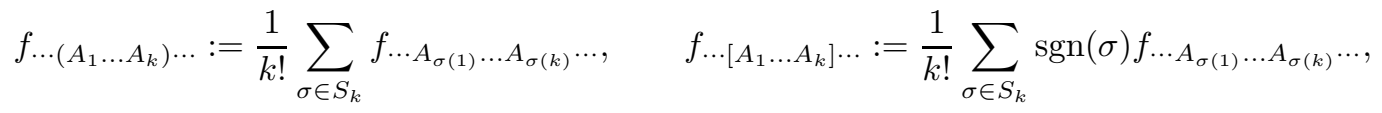

where $\operatorname{sgn}(\sigma)$ is the sign of the permutation $\sigma$. It is obvious that

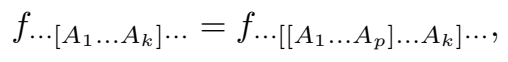

by definition of antisymmetrisation. We will also us the notation

$$
f \ldots\left[A_{1} \ldots|\mathscr{A}| \ldots A_{k}\right] \ldots,
$$

which means antisymmetrisation of indices $A_{1} \ldots A_{k}$ except for that in $\mathscr{A}$. We will use similar notations for symmetrisation of primed indices. 


\subsection{The curvature decomposition on unimodular quaternionic manifolds.}

Proposition 2.2. For a unimodular quaternionic manifold with dimension $>4$, the curvatures decompose as

$$
\begin{aligned}
R_{\left[A^{\prime} B^{\prime}\right] A B C}{ }^{D} & =\varepsilon_{A^{\prime} B^{\prime}}\left(\Psi_{A B C}{ }^{D}+2 \delta_{(A}{ }^{D} \Lambda_{B) C}\right), \\
R_{\left(A^{\prime} B^{\prime}\right) A B C}{ }^{D} & =2 \delta_{[A}{ }^{D} \Phi_{B] C A^{\prime} B^{\prime}}, \\
R_{(A B) A^{\prime} B^{\prime} C^{\prime}}{ }^{D^{\prime}} & =2 \delta_{\left[A^{\prime}\right.}{ }^{D^{\prime}} \Phi_{\left.B^{\prime}\right] C^{\prime} A B}, \\
R_{[A B] A^{\prime} B^{\prime} C^{\prime}}{ }^{D^{\prime}} & =2 \Lambda_{A B} \delta_{\left(A^{\prime}\right.}{ }^{D^{\prime}} \varepsilon_{\left.B^{\prime}\right) C^{\prime}},
\end{aligned}
$$

where the first identity above can viewed as the definition of $\Psi_{A B C}{ }^{D}$, and

$$
\varepsilon_{A^{\prime} B^{\prime}} \Lambda_{A B}:=\frac{1}{3} R_{[A B] C^{\prime}\left[A^{\prime} B^{\prime}\right]}^{C^{\prime}}, \quad \Phi_{A B A^{\prime} B^{\prime}}:=R_{(A B) C^{\prime}\left(A^{\prime} B^{\prime}\right)} C^{\prime},
$$

and $\Phi_{A^{\prime} B^{\prime} A B}:=\Phi_{A B A^{\prime} B^{\prime}}$. Moreover,

$$
\Lambda_{A B}=\Lambda_{[A B]}, \quad \Phi_{A B A^{\prime} B^{\prime}}=\Phi_{(A B)\left(A^{\prime} B^{\prime}\right)}, \quad \Psi_{A B C}{ }^{D}=\Psi_{(A B C)}{ }^{D}
$$

and $\Psi_{A B C}{ }^{D}$ are totally trace free: $\Psi_{A B C}{ }^{A}=\Psi_{A B C}{ }^{B}=\Psi_{A B C}{ }^{C}=0$.

When the manifold is 4-dimensional, 2.24) holds except for the last identity replaced by

$$
R_{[A B] A^{\prime} B^{\prime} C^{\prime}}^{D^{\prime}}=\epsilon_{A B} \Psi_{A^{\prime} B^{\prime} C^{\prime}}^{D^{\prime}}+2 \Lambda_{A B} \delta_{\left(A^{\prime}\right.}^{D^{\prime}} \varepsilon_{\left.B^{\prime}\right) C^{\prime}},
$$

with $\Psi_{A^{\prime} B^{\prime} C^{\prime}}^{\prime D^{\prime}}=\Psi_{\left(A^{\prime} B^{\prime} C^{\prime}\right)}^{\prime} D^{\prime}$ also totally trace free. (2.27) can be viewed as the definition of $\Psi^{\prime}$.

In the 4-dimensional case, we will only consider right conformally flat manifolds later, i.e. $\Psi_{A^{\prime} B^{\prime} C^{\prime}}^{D^{\prime}}=$ 0 (cf. section 6.9 of [31] for this concept and its necessity for defining massless field equations). See Penrose and Rindler's book (section 4.6 of [30]) for this curvature decomposition for 4-dimensional manifolds. It is generalized to torsion-free QCFs by Bailey and Eastwood (cf. p.83 in [5]) with a sketched proof. See the Appendix for a detailed proof by only using the first Bianchi identity.

It is well known that a quaternionic Kähler manifold is Einstein. See lemma A.1 and theorem 7.8 in [5] for the proofs of the following proposition for QCF manifolds. See also the Appendix for a detailed proof.

Proposition 2.3. (1) If the manifold is unimodular quaternionic, then we have $\nabla_{[A}^{A^{\prime} \Lambda_{B C]}}=0$.

(2) For a quaternionic Kähler manifold, we have

$$
\Phi_{A B A^{\prime} B^{\prime}}=0, \quad \Lambda_{A B}=\Lambda \epsilon_{A B}, \quad \Lambda=\frac{s_{g}}{8 n(n+2)},
$$

where $s_{g}$ is the scalar curvature. Namely, it is Einstein.

\section{THE QUATERNIONIC COMPLEXES}

3.1. The $k$-Cauchy-Fueter complexes. Recall that an element of the symmetric power $\odot^{p} H^{*}$ is given by a tuple $\left(f_{A_{1}^{\prime} \ldots A_{p}^{\prime}}\right)$, which as an element of $\otimes^{p} H^{*}$ is invariant under the permutation of subscripts $A_{1}^{\prime}, \ldots, A_{p}^{\prime}=0^{\prime}, 1^{\prime}$. An element of the exterior power $\Lambda^{q} E^{*}$ is given by a tuple $\left(f_{A_{1} \ldots A_{q}}\right)$, which as an element of $\otimes^{q} E^{*}$ is antisymmetric under the permutation of subscripts $A_{1}, \ldots, A_{q}=0, \ldots, 2 n-1$.

The covariant derivative defines a differential operator $\nabla: \Gamma\left(\mathfrak{T}_{q, p}\right) \rightarrow \Gamma\left(\mathfrak{T}_{q+1, p+1}\right)$ given by

$$
(\nabla f)_{A_{0} \ldots A_{q} A_{0}^{\prime} \ldots A_{p}^{\prime}}=\nabla_{A_{0} A_{0}^{\prime}} f_{A_{1} \ldots A_{q} A_{1}^{\prime} \ldots A_{p}^{\prime}}
$$


Note that for $f \in \Gamma\left(\Lambda^{q} E^{*} \otimes \odot{ }^{p} H^{*}\right),(\nabla f)_{A_{0} \ldots A_{q} A_{0}^{\prime} A_{1}^{\prime} \ldots A_{p}^{\prime}}$ is still symmetric in $A_{1}^{\prime}, \ldots, A_{p}^{\prime}$ and antisymmetric in $A_{1}, \ldots, A_{p}$ by using the formula (2.15) for covariant derivatives. We need its antisymmetrisation $\widehat{\nabla}: \Gamma\left(\mathfrak{T}_{q, p}\right) \rightarrow \Gamma\left(\Lambda^{q+1} E^{*} \otimes\left(\otimes^{p+1} H^{*}\right)\right)$ given by

$$
(\widehat{\nabla} f)_{A_{0} \ldots A_{q} A_{0}^{\prime} \ldots A_{p}^{\prime}}=\nabla_{A_{0}^{\prime}\left[A_{0}\right.} f_{\left.A_{1} \ldots A_{q}\right] A_{1}^{\prime} \ldots A_{p}^{\prime}} .
$$

Let us consider operators a little bit more general than those appearing in the $k$-Cauchy-Fueter complexes (1.3): $\mathscr{D}_{q, p}: \Gamma\left(\Lambda^{q} E^{*} \otimes \odot^{p} H^{*}\right) \longrightarrow \Gamma\left(\Lambda^{q+1} E^{*} \otimes \odot^{p-1} H^{*}\right)$ given by

$$
\left(\mathscr{D}_{q, p} f\right)_{A_{1} \ldots A_{q+1} A_{2}^{\prime} \ldots A_{p}^{\prime}}=\nabla_{\left[A_{1}\right.}^{A_{1}^{\prime}} f_{\left.A_{2} \ldots A_{q+1}\right] A_{1}^{\prime} A_{2}^{\prime} \ldots A_{p}^{\prime}},
$$

for a local section $f$ in $\Gamma\left(\Lambda^{q} E^{*} \otimes \odot^{p} H^{*}\right)$ (it is well defined since contraction over $A_{1}^{\prime}$ is well defined), and $\mathscr{D}_{q}^{p}: \Gamma\left(\Lambda^{q} E^{*} \otimes \odot^{p} H\right) \longrightarrow \Gamma\left(\Lambda^{q+1} E^{*} \otimes \odot^{p+1} H\right)$ given by

$$
\left(\mathscr{D}_{q}^{p} f\right)_{A_{1} \ldots A_{q+1}}^{A_{1}^{\prime} \ldots A_{p+1}^{\prime}}=\nabla_{\left[A_{1}\right.}^{\left(A_{1}^{\prime}\right.} f_{\left.A_{2} \ldots A_{q+1}\right]}^{\left.A_{2}^{\prime} \ldots A_{p+1}^{\prime}\right)}
$$

for a local section $f$ in $\Gamma\left(\Lambda^{q} E^{*} \otimes \odot^{p} H^{*}\right)$. The Baston operator $\triangle: \Gamma\left(\Lambda^{k} E^{*}\right) \longrightarrow \Gamma\left(\Lambda^{k+2} E^{*}\right)$ is given by

$$
(\triangle f)_{A_{1} \cdots A_{k+2}}:=\nabla_{A^{\prime}\left[A_{1}\right.} \nabla_{A_{2}}^{A^{\prime}} f_{\left.A_{3} \cdots A_{k+2}\right]}+2 \Lambda_{\left[A_{1} A_{2}\right.} f_{\left.A_{3} \cdots A_{k+2}\right]} .
$$

Theorem 3.1. Suppose that the manifold $M$ is unimodular quaternionic and is right conformally flat if $\operatorname{dim}_{\mathbb{R}} M=4$. For $k=0,1,2, \ldots$, the sequences (1.3) are elliptic differential complexes, where the operators $D_{j}^{(k)}=\mathscr{D}_{j, k-j}$ for $j=0, \ldots, k-1$, the operators $D_{j}^{(k)}=\mathscr{D}_{j+1}^{j-k-1}$ for $j=k+1, \ldots, 2 n-2$, and the operator $D_{k}^{(k)}$ is the Baston operator $\triangle$.

Note that by using $\varepsilon^{A^{\prime} B^{\prime}}$ (2.19) to raise primed indices, we have the following commutators

$$
\begin{aligned}
& \left(\nabla_{A}^{A^{\prime}} \nabla_{B}^{B^{\prime}}-\nabla_{B}^{B^{\prime}} \nabla_{A}^{A^{\prime}}\right) f_{C^{\prime}}=\left(\nabla_{A \widetilde{A^{\prime}}} \nabla_{B \widetilde{B}^{\prime}}-\nabla_{B \widetilde{B}^{\prime}} \nabla_{A \widetilde{A^{\prime}}}\right) f_{C^{\prime}} \varepsilon^{\widetilde{A}^{\prime} A^{\prime}} \varepsilon^{\widetilde{B}^{\prime} B^{\prime}} \\
& =-R_{A B \widetilde{A^{\prime}} \widetilde{B}^{\prime} C^{\prime}}{ }^{D^{\prime}} f_{D^{\prime}} \varepsilon^{\widetilde{A}^{\prime} A^{\prime}} \varepsilon^{\widetilde{B}^{\prime} B^{\prime}}=-R_{A B}{ }^{A^{\prime} B^{\prime}} C_{C^{\prime}}^{D^{\prime}} f_{D^{\prime}}, \\
& \left(\nabla_{A}^{A^{\prime}} \nabla_{B}^{B^{\prime}}-\nabla_{B}^{B^{\prime}} \nabla_{A}^{A^{\prime}}\right) f_{C}=-R^{A^{\prime} B^{\prime}}{ }_{A B C}{ }^{D} f_{D},
\end{aligned}
$$

where $R^{A^{\prime} B^{\prime}}{ }_{A B C}{ }^{D}:=R_{\widetilde{A^{\prime}} \widetilde{B}^{\prime} A B C}{ }^{D} \varepsilon^{\widetilde{A}^{\prime} A^{\prime}} \varepsilon^{\widetilde{B}^{\prime} B^{\prime}}$ by raising indices. We can move $\varepsilon^{\widetilde{A}^{\prime} A^{\prime}}$ to the left since the connection $\nabla$ preserves it. The following formulae for commutators $\nabla_{[A}^{\left(A^{\prime}\right.} \nabla_{B]}^{\left.B^{\prime}\right)}$ as curvatures are important in the proof of Theorem 3.1

$$
\begin{aligned}
\nabla_{\left[A_{1}\right.}^{\left(A_{1}^{\prime}\right.} \nabla_{\left.A_{2}\right]}^{\left.A_{2}^{\prime}\right)} h_{C^{\prime}} & =\frac{1}{4}\left(\nabla_{A_{1}}^{A_{1}^{\prime}} \nabla_{A_{2}}^{A_{2}^{\prime}}-\nabla_{A_{2}}^{A_{2}^{\prime}} \nabla_{A_{1}}^{A_{1}^{\prime}}-\nabla_{A_{2}}^{A_{1}^{\prime}} \nabla_{A_{1}}^{A_{2}^{\prime}}+\nabla_{A_{1}}^{A_{2}^{\prime}} \nabla_{A_{2}}^{A_{1}^{\prime}}\right) h_{C^{\prime}}=-\frac{1}{2} R_{\left[A_{1} A_{2}\right]}{ }^{A_{1}^{\prime} A_{2}^{\prime}{ }^{\prime}{ }^{\prime}{ }^{\prime} h_{D^{\prime}},} \\
\nabla_{\left[A_{1}\right.}^{\left(A_{1}^{\prime}\right.} \nabla_{\left.A_{2}\right]}^{\left.A_{2}^{\prime}\right)} h_{C} & =-\frac{1}{2} R_{A_{1} A_{2} C}^{\left(A_{1}^{\prime} A_{2}^{\prime}\right)} h_{D} .
\end{aligned}
$$

by using (3.4). We will also frequently use the following corollary of Proposition 2.2 .

Corollary 3.1. On a unimodular quaternionic manifold (right conformally flat if it is 4-dimensional), we have

$$
R_{\left[A_{1} A_{2}\right]}^{A^{\prime} B^{\prime}}{ }_{C^{\prime}}^{D^{\prime}}=2 \Lambda_{A_{1} A_{2}} \delta_{C^{\prime}}{ }^{\left(A^{\prime}\right.} \varepsilon^{\left.B^{\prime}\right) D^{\prime}},
$$

in particular,

$$
R_{\left[A_{1} A_{2}\right]}^{\left(A^{\prime} B^{\prime} \quad D^{\prime}\right)}=0
$$

and

$$
R_{A_{1}^{\prime} A_{2}^{\prime}[A B C]}^{D}=0, \quad \text { and } \quad R_{A B C}^{\left(A^{\prime} B^{\prime}\right)}{ }^{D}=2 \delta_{[A}^{D} \Phi_{B] C}{ }^{\left(A^{\prime} B^{\prime}\right)} .
$$


Proof. (3.6) follows from

$$
R_{\left[A_{1} A_{2}\right]}{ }^{A^{\prime} B^{\prime}}{ }_{C^{\prime}}^{D^{\prime}}=R_{\left[A_{1} A_{2}\right] \widetilde{A^{\prime}} \widetilde{B}^{\prime} C^{\prime}}{ }^{D^{\prime}} \varepsilon^{\widetilde{A}^{\prime} A^{\prime}} \varepsilon^{\widetilde{B}^{\prime} B^{\prime}}=2 \Lambda_{A_{1} A_{2}} \delta_{\left(\widetilde{A}^{\prime}\right.}^{D^{\prime}} \varepsilon_{\left.\widetilde{B}^{\prime}\right) C^{\prime}} \varepsilon^{\widetilde{A}^{\prime} A^{\prime}} \varepsilon^{\widetilde{B}^{\prime} B^{\prime}}=2 \Lambda_{A_{1} A_{2}} \delta_{C^{\prime}}{ }^{\left(A^{\prime}\right.} \varepsilon^{\left.B^{\prime}\right) D^{\prime}},
$$

by the last identity in (2.24) and $\varepsilon^{D^{\prime} A^{\prime}}$ antisymmetric.

The first identity in (3.8) follows from antisymmetrising $[A B C]$ in

$$
R_{A_{1}^{\prime} A_{2}^{\prime} A B C}{ }^{D}=\varepsilon_{A_{1}^{\prime} A_{2}^{\prime}}\left(\Psi_{A B C}{ }^{D}+2 \delta_{(A}{ }^{D} \Lambda_{B) C}\right)+2 \delta_{[A}{ }^{D} \Phi_{B] C A_{1}^{\prime} A_{2}^{\prime}},
$$

by using (2.24) and symmetry (2.26) of $\Phi$ and $\Psi$ in subscripts. For the second identity, we have

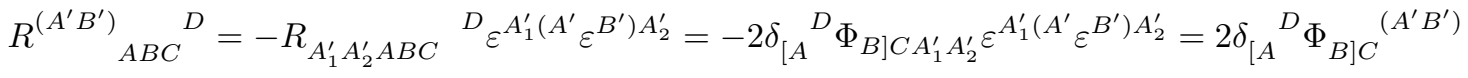

by using (3.9) and $\varepsilon_{A_{1}^{\prime} A_{2}^{\prime}} \varepsilon^{A_{1}^{\prime}\left(A^{\prime}\right.} \varepsilon^{\left.B^{\prime}\right) A_{2}^{\prime}}=-\varepsilon^{\left(B^{\prime} A^{\prime}\right)}=0$.

Proof of Theorem 3.1 Case 1: $j=0, \ldots, k-2$. To check $D_{j+1}^{(k)} \circ D_{j}^{(k)}=0$, it is sufficient to show $\mathscr{D}_{q+1, p-1} \mathscr{D}_{q, p}=0$. For a local section $f \in \Gamma\left(\Lambda^{q} E^{*} \otimes \odot{ }^{p} H^{*}\right)$, we have

$$
\left(\mathscr{D}_{q+1, p-1} \mathscr{D}_{q, p} f\right)_{A_{1} \ldots A_{q+2} A_{3}^{\prime} \ldots A_{p}^{\prime}}=\nabla_{\left[A_{1}\right.}^{A_{1}^{\prime}} \nabla_{\left[A_{2}\right.}^{A_{2}^{\prime}} f_{\left.\left.A_{3} \ldots A_{q+2}\right]\right] A_{2}^{\prime} A_{1}^{\prime} A_{3}^{\prime} \ldots A_{p}^{\prime}}=\nabla_{\left[\left[A_{1}\right.\right.}^{\left(A_{1}^{\prime}\right.} \nabla_{\left.A_{2}\right]}^{\left.A_{2}^{\prime}\right)} f_{\left.A_{3} \ldots A_{q+2}\right] A_{1}^{\prime} A_{2}^{\prime} \ldots A_{p}^{\prime}} .
$$

by (2.23). We can symmetrise superscripts $\left(A_{1}^{\prime} A_{2}^{\prime}\right)$ since $\nabla_{b} f \ldots A_{1}^{\prime} A_{2}^{\prime} \ldots A_{p}^{\prime}$ is symmetric in $A_{1}^{\prime}, \ldots, A_{p}^{\prime}$ as we have mentioned under (3.1), and so is $\nabla_{a} \nabla_{b} f \ldots A_{1}^{\prime} A_{2}^{\prime} \ldots A_{p}^{\prime}$. Apply formula (3.5) for commutators to the above identity and antisymmetrise unprimed indices to get $\left(\mathscr{D}_{q+1, p-1} \mathscr{D}_{q, p} f\right)_{A_{1} \ldots A_{q+2} A_{3}^{\prime} \ldots A_{p}^{\prime}}$ equal to

$$
-\frac{1}{2} R_{\left[\left[A_{1} A_{2}\right]\right.}^{A_{1}^{\prime} A_{2}^{\prime}}{ }_{\left|A_{j}^{\prime}\right|}^{D^{\prime}} f_{\left.A_{3} \ldots A_{q+2}\right] \ldots D^{\prime} \ldots}-\frac{1}{2} R_{\left[\left[A_{1} A_{2} A_{j}\right]\right.}^{\left.{ }^{D} A_{1}^{\prime} A_{2}^{\prime}\right)} f_{\left.A_{3} \ldots|D| \ldots A_{q+2}\right] A_{1}^{\prime} A_{2}^{\prime} \ldots A_{p}^{\prime}},
$$

by using (2.23). The second term in (3.10) vanishes by (3.8), while the first term in (3.10) is equal to

$$
\begin{aligned}
& -\frac{3}{2}\left\{\varepsilon^{A_{2}^{\prime} D^{\prime}} \Lambda_{\left[A_{1} A_{2}\right.} f_{\left.A_{3} \ldots A_{q+2}\right] D^{\prime} A_{2}^{\prime} \ldots A_{p}^{\prime}}+\varepsilon^{A_{1}^{\prime} D^{\prime}} \Lambda_{\left[A_{1} A_{2}\right.} f_{\left.A_{3} \ldots A_{q+2}\right] A_{1}^{\prime} D^{\prime} A_{3}^{\prime} \ldots}\right\} \\
& -\frac{1}{2} R_{\left[A_{1} A_{2}\right.}^{A_{1}^{\prime} A_{2}^{\prime}} \stackrel{D^{\prime}}{\left|A_{j}^{\prime}\right|} f_{\left.A_{3} \ldots A_{q+2}\right] A_{1}^{\prime} A_{2}^{\prime} \ldots D^{\prime} \ldots A_{p}^{\prime}}=0
\end{aligned}
$$

by (3.6)-(3.7), since $f$ is symmetric in primed indices. Consequently, we get $\mathscr{D}_{q+1, p-1} \mathscr{D}_{q, p} f=0$.

Case 2: $j=k+1, \ldots, 2 n-2$. To check $D_{j+1}^{(k)} \circ D_{j}^{(k)}=0$, it is sufficient to show $\mathscr{D}_{q+1}^{p+1} \mathscr{D}_{q}^{p}=0$. Similarly, we have

$$
\begin{aligned}
\left(\mathscr{D}_{q+1}^{p+1} \mathscr{D}_{q}^{p} f\right)_{A_{1} \ldots A_{q+2}}^{A_{1}^{\prime} \ldots A_{p+2}^{\prime}} & =\nabla_{\left[A_{1}\right.}^{\left(A_{1}^{\prime}\right.} \nabla_{\left[A_{2}\right.}^{\left(A_{2}^{\prime}\right.} f_{\left.\left.A_{3} \ldots A_{q+2}\right]\right]}^{\left.\left.A_{3}^{\prime} \ldots A_{p+2}^{\prime}\right)\right)}=\nabla_{\left[\left[A_{1}\right.\right.}^{\left(\left(A_{1}^{\prime}\right.\right.} \nabla_{\left.A_{2}\right]}^{\left.A_{2}^{\prime}\right)} f_{\left.A_{3} \ldots A_{q+2}\right]}^{\left.A_{3}^{\prime} \ldots A_{p+2}^{\prime}\right)} \\
& =\frac{1}{2} R_{\left[A_{1} A_{2}\right.}{ }^{\left(A_{1}^{\prime} A_{2}^{\prime}\right.}{ }_{\left|D^{\prime}\right|}^{A_{j}^{\prime}} f_{\left.A_{3} \ldots A_{q+2}\right]}^{\left.A_{3}^{\prime} \ldots\left|D^{\prime}\right| \ldots A_{p+2}^{\prime}\right)}-\frac{1}{2} R_{\left[A_{1} A_{2} A_{j}\right.}^{\left(A_{1}^{\prime} A_{2}^{\prime}\right.} f_{\left.A_{3} \ldots|D| \ldots A_{q+2}\right]}^{\left.A_{3}^{\prime} \ldots A_{p+2}^{\prime}\right)}=0
\end{aligned}
$$

by using (3.5) and (3.7)-(3.8) again for vanishing of curvatures.

Case 3: $j=k-1$. Recall that $\Gamma\left(\Lambda^{k-1} E^{*} \otimes H^{*}\right) \stackrel{\mathscr{D}_{k-1,1}}{\longrightarrow} \Gamma\left(\Lambda^{k} E^{*}\right) \stackrel{\triangle}{\longrightarrow} \Gamma\left(\Lambda^{k+2} E^{*}\right) \stackrel{\mathscr{D}_{k+2}^{0}}{\longrightarrow} \Gamma\left(\Lambda^{k+3} E^{*}\right.$ $\otimes H)$. Let us show that for a local section $f \in \Gamma\left(\Lambda^{k-1} E^{*} \otimes H^{*}\right)$,

$$
\left(\Delta \mathscr{D}_{k-1,1} f\right)_{A_{1} \ldots A_{k+2}}=\nabla_{A^{\prime}\left[A_{1}\right.} \nabla_{A_{2}}^{A^{\prime}} \nabla_{A_{3}}^{B^{\prime}} f_{\left.A_{4} \ldots A_{k+2}\right] B^{\prime}}+2 \Lambda_{\left[A_{1} A_{2}\right.} \nabla_{A_{3}}^{B^{\prime}} f_{\left.A_{4} \ldots A_{k+2}\right] B^{\prime}}=0 .
$$

Locally we choose a coordinate chart $U_{\alpha}$ with trivialization $\left.E^{*}\right|_{U_{\alpha}}=U_{\alpha} \times \mathbb{C}^{2 n},\left.H^{*}\right|_{U_{\alpha}}=U \times \mathbb{C}^{2}$, and a two-component local quaternionic frame $\left\{Z_{A A^{\prime}}\right\}$ such that $\varepsilon$ and $\epsilon$ are standard. In particular, 
$\varepsilon^{1^{\prime} 0^{\prime}}=-\varepsilon^{0^{\prime} 1^{\prime}}=1$. Note that

$$
\begin{aligned}
\nabla_{A^{\prime}\left[A_{1}\right.} \nabla_{A_{2}}^{A^{\prime}} \nabla_{A_{3}}^{B^{\prime}} f_{\left.A_{4} \ldots A_{k+2}\right] B^{\prime}}= & \nabla_{0^{\prime}\left[A_{1}\right.} \nabla_{A_{2}}^{0^{\prime}} \nabla_{A_{3}}^{0^{\prime}} f_{\left.A_{4} \ldots A_{k+2}\right] 0^{\prime}}+\nabla_{1^{\prime}\left[A_{1}\right.} \nabla_{A_{2}}^{1^{\prime}} \nabla_{A_{3}}^{0^{\prime}} f_{\left.A_{4} \ldots A_{k+2}\right] 0^{\prime}} \\
& +\nabla_{0^{\prime}\left[A_{1}\right.} \nabla_{A_{2}}^{0^{\prime}} \nabla_{A_{3}}^{1^{\prime}} f_{\left.A_{4} \ldots A_{k+2}\right] 1^{\prime}}+\nabla_{1^{\prime}\left[A_{1}\right.} \nabla_{A_{2}}^{1^{\prime}} \nabla_{A_{3}}^{1^{\prime}} f_{\left.A_{4} \ldots A_{k+2}\right] 1^{\prime}} \\
= & : \Sigma_{1}+\Sigma_{2}+\Sigma_{3}+\Sigma_{4},
\end{aligned}
$$

where both sides are sections of $\mathfrak{T}_{k+2,2}^{2}$, while the left hand side can be viewed as a section of $\mathfrak{T}_{k+2,0}^{0}$ after contraction of two primed indices. Since

$$
\nabla_{[A}^{A^{\prime}} \nabla_{B]}^{A^{\prime}} f \ldots=\frac{1}{2}\left(\nabla_{A}^{A^{\prime}} \nabla_{B}^{A^{\prime}}-\nabla_{B}^{A^{\prime}} \nabla_{A}^{A^{\prime}}\right) f_{\ldots}
$$

we have

$$
\begin{aligned}
\Sigma_{1} & =\nabla_{0^{\prime}\left[A_{1}\right.} \nabla_{\left[A_{2}\right.}^{0^{\prime}} \nabla_{\left.A_{3}\right]}^{0^{\prime}} f_{\left.A_{4} \ldots A_{k+2}\right] 0^{\prime}} \\
& =\frac{1}{2} \nabla_{0^{\prime}\left[A_{1}\right.}\left(-R_{\left[A_{2} A_{3}\right]}^{0^{\prime} 0^{\prime}} \quad A^{0^{\prime} \mid} f_{\left.A_{4} \ldots A_{k+2}\right] A^{\prime}}-R^{0^{\prime} 0^{\prime}}{ }_{\left[A_{2} A_{3} A_{j}\right]}^{D} f_{\left.A_{4} \ldots|D| \ldots A_{k+2}\right] 0^{\prime}}\right) \\
& =\nabla_{0^{\prime}\left[A_{1}\right.}\left(\Lambda_{A_{2} A_{3}} f_{\left.A_{4} \ldots A_{k+2}\right] 1^{\prime}}\right)=-\Lambda_{\left[A_{2} A_{3}{ }_{3} \nabla_{A_{1}}^{1^{\prime}} f_{\left.A_{4} \ldots A_{k+2}\right] 1^{\prime}},\right.}
\end{aligned}
$$

by using (3.5)-(3.6), $R_{\left[A_{2} A_{3} A_{j}\right]}^{D}=0$ by (3.8) and Proposition 2.3 (1). Similarly, we have

$$
\begin{aligned}
\Sigma_{4} & =\frac{1}{2} \nabla_{1^{\prime}\left[A_{1}\right.}\left(-R_{\left[A_{2} A_{3}\right]}^{1^{\prime} 1^{\prime}}{ }_{\left|1^{\prime}\right|}^{A^{\prime}} f_{\left.A_{4} \ldots A_{m+2}\right] A^{\prime}}-R^{1^{\prime} 1^{\prime}}{ }_{\left[A_{2} A_{3} A_{j}\right.}^{D} f_{\left.A_{4} \ldots|D| \ldots A_{k+2}\right] 1^{\prime}}\right) \\
& =\nabla_{1^{\prime}\left[A_{1}\right.}\left(-\Lambda_{A_{2} A_{3}} f_{\left.A_{4} \ldots A_{k+2}\right] 0^{\prime}}\right)=-\Lambda_{\left[A_{2} A_{3}\right.} \nabla_{A_{1}}^{0^{\prime}} f_{\left.A_{4} \ldots A_{k+2}\right] 0^{\prime}} .
\end{aligned}
$$

To calculate the term $\Sigma_{2}$, applying the trivial identity

$$
\nabla_{A}^{0^{\prime}} \nabla_{B}^{1^{\prime}} f_{\ldots}=\left[\nabla_{B}^{1^{\prime}} \nabla_{A}^{0^{\prime}}+\left(\nabla_{A}^{0^{\prime}} \nabla_{B}^{1^{\prime}}-\nabla_{B}^{1^{\prime}} \nabla_{A}^{0^{\prime}}\right)\right] f_{\ldots}
$$

and (3.4) for commutators, then antisymmetrising $\left[A_{1} \ldots A_{k+2}\right]$, we get

$$
\begin{aligned}
\Sigma_{2}= & \nabla_{\left[A_{1}\right.}^{0^{\prime}} \nabla_{A_{2}}^{1^{\prime}} \nabla_{A_{3}}^{0^{\prime}} f_{\left.A_{4} \ldots A_{k+2}\right] 0^{\prime}} \\
= & \nabla_{\left[A_{2}\right.}^{1^{\prime}} \nabla_{A_{1}}^{0^{\prime}} \nabla_{A_{3}}^{0^{\prime}} f_{\left.A_{4} \ldots A_{k+2}\right] 0^{\prime}}-R^{0^{\prime} 1^{\prime}} \quad{ }_{\left[A_{1} A_{2} A_{j}\right.}{ }^{D} \nabla_{A_{3}}^{0^{\prime}} f_{\left.\ldots|D| \ldots A_{k+2}\right] 0^{\prime}} \\
& +R_{\left[A_{1} A_{2}\right.}{ }^{0^{\prime} 1^{\prime}} \quad{ }^{\prime}{ }^{\prime}{ }^{\prime} \nabla_{A_{3}}^{A^{\prime}} f_{\left.A_{4} \ldots A_{k+2}\right] 0^{\prime}}-R_{\left[A_{1} A_{2}\right.}{ }^{0_{1} 1^{\prime}} \quad{ }^{0^{\prime} \mid} \nabla_{A_{3}}^{0^{\prime}} f_{\left.A_{4} \ldots A_{k+2}\right] A^{\prime}} \\
= & -\nabla_{\left[A_{1}\right.}^{1^{\prime}} \nabla_{A_{2}}^{0^{\prime}} \nabla_{A_{3}}^{0^{\prime}} f_{\left.A_{4} \ldots A_{k+2}\right] 0^{\prime}}=\Sigma_{1},
\end{aligned}
$$

(here $D$ is in the $j$-th place of $A_{3}, \ldots A_{k+2}$ ), since the last two curvature terms cancel by

$$
R_{\left[A_{1} A_{2}\right]}{ }^{0^{\prime} 1^{\prime}}{ }_{A^{\prime}}^{0^{\prime}}=\Lambda_{A_{1} A_{2}} \delta_{A^{\prime}}{ }^{0^{\prime}} \varepsilon^{1^{\prime} 0^{\prime}}=R_{\left[A_{1} A_{2}\right]}{ }^{0^{\prime} 1^{\prime}}{ }^{0^{\prime}} A^{\prime},
$$

and using (3.6) again. Similarly, we have

$$
\begin{aligned}
& \Sigma_{3}=-\nabla_{\left[A_{1}\right.}^{1^{\prime}} \nabla_{A_{2}}^{0^{\prime}} \nabla_{A_{3}}^{1^{\prime}} f_{\left.A_{4} \ldots A_{k+2}\right] 1^{\prime}} \\
& =-\nabla_{\left[A_{2}\right.}^{0^{\prime}} \nabla_{A_{1}}^{1^{\prime}} \nabla_{A_{3}}^{1^{\prime}} f_{\left.A_{4} \ldots A_{k+2}\right] 1^{\prime}}+R^{1^{\prime} 0^{\prime}}{ }_{\left[A_{1} A_{2} A_{j}\right.}^{D} \nabla_{A_{3}}^{1^{\prime}} f_{\left.A_{4} \ldots|D| \ldots A_{k+2}\right] 1^{\prime}} \\
& -R_{\left[A_{1} A_{2}{ }^{1^{\prime} 0^{\prime}}\left|{ }^{\prime}\right|\right.}{ }^{1^{\prime}} \nabla_{A_{3}}^{A^{\prime}} f_{\left.A_{4} \ldots A_{k+2}\right] 1^{\prime}}+R_{\left[A_{1} A_{2}\right.}{ }^{1^{\prime} 0^{\prime}}{ }_{\left|1^{\prime}\right|}^{A^{\prime}} \nabla_{A_{3}}^{1^{\prime}} f_{\left.A_{4} \ldots A_{k+2}\right] A^{\prime}} \\
& =\nabla_{\left[A_{1}\right.}^{0^{\prime}} \nabla_{A_{2}}^{1^{\prime}} \nabla_{A_{3}}^{1^{\prime}} f_{\left.A_{4} \ldots A_{m+2}\right] 1^{\prime}}=\Sigma_{4} \text {. }
\end{aligned}
$$

Thus, we find that $\Sigma_{1}+\Sigma_{2}+\Sigma_{3}+\Sigma_{4}=-2 \Lambda_{\left[A_{1} A_{2}\right.} \nabla_{A_{3}}^{A^{\prime}} f_{\left.A_{4} \ldots A_{k+2}\right] A^{\prime}}$. So (3.12) follows. 
Case 4: $j=k$. For $k \geq 1$ and $B^{\prime}=0^{\prime}$, we have

$$
\begin{aligned}
& \left(\mathscr{D}_{k+2}^{0} \Delta f\right)_{A_{1} \ldots A_{k+3}}^{B^{\prime}}=\nabla_{\left[A_{1}\right.}^{B^{\prime}} \nabla_{\left|A^{\prime}\right| A_{2}} \nabla_{A_{3}}^{A^{\prime}} f_{\left.A_{4} \ldots A_{k+3}\right]}+2 \nabla_{\left[A_{1}\right.}^{B^{\prime}}\left(\Lambda_{A_{2} A_{3}} f_{\left.A_{4} \ldots A_{k+3}\right]}\right) \\
& \quad=\nabla_{\left[A_{1}\right.}^{0^{\prime}} \nabla_{A_{2}}^{0^{\prime}} \nabla_{A_{3}}^{1^{\prime}} f_{\left.A_{4} \ldots A_{m+2}\right]}-\nabla_{\left[A_{1}\right.}^{0^{\prime}} \nabla_{A_{2}}^{1^{\prime}} \nabla_{A_{3}}^{0^{\prime}} f_{\left.A_{4} \ldots A_{k+3}\right]}+2 \Lambda_{\left[A_{2} A_{3}\right.} \nabla_{A_{1}}^{0^{\prime}} f_{\left.A_{4} \ldots A_{k+3}\right]} \\
& \quad=2 \nabla_{\left[\left[A_{1}\right.\right.}^{0^{\prime}} \nabla_{\left.A_{2}\right]}^{0^{\prime}} \nabla_{A_{3}}^{1^{\prime}} f_{\left.A_{4} \ldots A_{m+2}\right]}+\nabla_{\left[A_{1}\right.}^{0^{\prime}} R^{1^{\prime} 0^{\prime}}{ }_{A_{2} A_{3} A_{j}}^{D}{ }_{A_{\left.A_{4} \ldots|D| \ldots A_{k+3}\right]}}+2 \Lambda_{\left[A_{2} A_{3}\right.} \nabla_{A_{1}}^{0^{\prime}} f_{\left.A_{4} \ldots A_{k+3}\right]}
\end{aligned}
$$

by raising the index $A^{\prime}$ and applying (3.15) to $\nabla_{A_{2}}^{1^{\prime}} \nabla_{A_{3}}^{0^{\prime}}$. The second term in the right hand side vanishes by (3.8), while the first term equals to

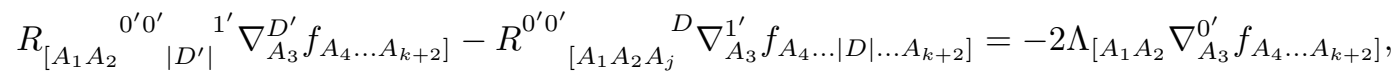

by using Corollary 3.1 again. So $\left(\mathscr{D}_{k+2}^{0} \Delta f\right)_{\ldots}^{B^{\prime}}$ vanishes for $B^{\prime}=0^{\prime}$. It is similar for $B^{\prime}=1^{\prime}$. If $k=0$, we can obtain the result by using vanishing of torsions.

The ellipiticity will be proved in Subsection 3.2.

Consider conformal transformation

$$
\widetilde{\epsilon}_{A_{1} \ldots A_{2 n}}:=\Omega \epsilon_{A_{1} \ldots A_{2 n}}, \quad \widetilde{\varepsilon}_{A^{\prime} B^{\prime}}:=\Omega \varepsilon_{A^{\prime} B^{\prime}}
$$

Fix a two-component local quaternionic frame $\left\{Z_{A A^{\prime}}\right\}$ with respect to a volume element vol in (2.9) and denote $\Upsilon_{A A^{\prime}}:=\Omega^{-1} Z_{A A^{\prime}} \Omega$. Under the conformal transformation (3.19), define a new connection $\widetilde{\nabla}$ on the bundles $E^{*}$ and $H^{*}$ by

$$
\begin{aligned}
\widetilde{\nabla}_{A A^{\prime}} f_{B} & =\nabla_{A A^{\prime}} f_{B}-\Theta_{A^{\prime} A B}{ }^{D} f_{D}, & & \Theta_{A^{\prime} A B}{ }^{D}=\delta_{A}{ }^{D} \Upsilon_{B A^{\prime}}, \\
\widetilde{\nabla}_{A A^{\prime}} f_{B^{\prime}} & =\nabla_{A A^{\prime}} f_{B^{\prime}}-\Theta_{A A^{\prime} B^{\prime}}{ }^{D^{\prime}} f_{D^{\prime}}, & & \Theta_{A A^{\prime} B^{\prime}}{ }^{D^{\prime}}=\delta_{A^{\prime}}{ }^{D^{\prime}} \Upsilon_{A B^{\prime}} .
\end{aligned}
$$

Then it is a quaternionic connection for the unimodular quaternionic structure with respect to the volume $\Omega^{2 n+2} \mathrm{vol}$. The curvatures of the unimodular quaternionic connection $\widetilde{\nabla}_{A A^{\prime}}$ satisfy

$$
\begin{aligned}
& \Omega \widetilde{\Lambda}_{A B}=\Lambda_{A B}+\frac{1}{2}\left(\nabla_{A^{\prime}[A} \Upsilon_{B]}^{A^{\prime}}+\Upsilon_{A^{\prime}[A} \Upsilon_{B]}^{A^{\prime}}\right), \quad \Omega \widetilde{\Psi}_{A B C}{ }^{D}=\Psi_{A B C}{ }^{D}, \\
& \widetilde{\Phi}_{A B A^{\prime} B^{\prime}}=\Phi_{A B A^{\prime} B^{\prime}}-\nabla_{\left(A \mid\left(A^{\prime}\right.\right.} \Upsilon_{\left.\left.B^{\prime}\right) \mid B\right)}+\Upsilon_{\left(A \mid\left(A^{\prime}\right.\right.} \Upsilon_{\left.\left.B^{\prime}\right) \mid B\right)} .
\end{aligned}
$$

Proposition 3.1. The operators associated to the unimodular quaternionic connection $\widetilde{\nabla}_{A A^{\prime}}$ are conformal covariant in the following sense:

$$
\begin{aligned}
& \widetilde{\mathscr{D}}_{q}^{p}\left(\Omega^{-q} f\right)=\Omega^{-q-1} \mathscr{D}_{q}^{p} f, \quad \text { for } \quad f \in \Gamma\left(\Lambda^{q} E^{*} \otimes \odot^{p} H\right), \\
& \widetilde{\mathscr{D}}_{q, p}\left(\Omega^{-q-1} f\right)=\Omega^{-q-2} \mathscr{D}_{q, p} f, \quad \text { for } \quad f \in \Gamma\left(\Lambda^{q} E^{*} \otimes \odot^{p} H^{*}\right) \text {, } \\
& \widetilde{\Delta}\left(\Omega^{-q-1} f\right)=\Omega^{-q-2} \Delta f, \quad \text { for } \quad f \in \Gamma\left(\Lambda^{q} E^{*}\right) \text {. }
\end{aligned}
$$

(3.22) holds with respect to fixed frame and coframe. The weight factors in (3.22) coincide with that obtained by the representation theory in Proposition 10 in Baston [6]. See Penrose and Rindler [30] section 5.6-5.7 for conformal transformation of spin $k / 2$ massless field operator over 4-dimensional manifolds. The conformal change of connections for QCF's was introduced by Bailey and Eastwood (cf. section 2.2 of [5]). See the Appendix for a detailed proof. 
3.2. The ellipticity of the $k$-Cauchy-Fueter complex. Locally for a matrix-valued differential operator $L=\sum_{j=0}^{m} \sum_{|\nu|=j} A_{\nu} \partial_{x_{\nu_{1}}} \cdots \partial_{x_{\nu_{j}}}$ of order $m$ on a $N$-dimensional manifold $M$, its symbol at point $p$ is

$$
\sigma(L)(\xi)=\sum_{|\nu|=m} A_{\nu}(p) \mathbf{i} \xi_{\nu_{1}} \cdots \mathbf{i} \xi_{\nu_{j}}, \quad \xi \in \mathbb{R}^{N}
$$

$L$ is called elliptic if $\sigma(L)(\xi)$ invertible for any $0 \neq \xi \in \mathbb{R}^{N}$. A differential complex is called elliptic if the associated symbol sequence is exact at each point $p$. It is well known that the ellipticity of a differential operator or a differential complex is independent of the choice of the local coordinate charts [49. For a fixed point $p \in M$, let $\sigma\left(X_{j}\right)=\mathbf{i} \xi_{j}$ and

$$
\left(\xi_{A A^{\prime}}\right):=\left(\begin{array}{lc}
\xi_{1}+\mathbf{i} \xi_{2} & -\xi_{3}-\mathbf{i} \xi_{4} \\
\xi_{3}-\mathbf{i} \xi_{4} & \xi_{1}-\mathbf{i} \xi_{2} \\
\vdots & \vdots \\
\xi_{4 n-3}+\mathbf{i} \xi_{4 n-2} & -\xi_{4 n-1}-\mathbf{i} \xi_{4 n} \\
\xi_{4 n-1}-\mathbf{i} \xi_{4 n} & \xi_{4 n-3}-\mathbf{i} \xi_{4 n-2}
\end{array}\right) .
$$

In particular, $\sigma\left(Z_{A A^{\prime}}\right)(\xi)=\frac{\mathbf{i}}{\sqrt{2}} \xi_{A A^{\prime}}$ by $Z_{A A^{\prime}}$ in (2.8). For fixed $k$, we use notations

$$
\sigma_{j}(\xi):=\frac{\sqrt{2}}{\mathbf{i}} \sigma\left(D_{j}^{(k)}\right)(\xi), \text { for } j \neq k, \quad \text { and } \quad \sigma_{k}(\xi):=\frac{2}{\mathbf{i}^{2}} \sigma\left(D_{k}^{(k)}\right)(\xi) .
$$

The ellipticity of the complex (1.3) is given by the following exact sequence of the associated symbols, which can be easily proved by using elementary linear algebra.

Proposition 3.2. For any $0 \neq \xi \in \mathbb{R}^{4 n}$,

$$
\begin{aligned}
0 \longrightarrow & \odot^{k} \mathbb{C}^{2} \stackrel{\sigma_{0}(\xi)}{\longrightarrow} \Lambda^{1} \mathbb{C}^{2 n} \otimes \odot^{k-1} \mathbb{C}^{2} \stackrel{\sigma_{1}(\xi)}{\longrightarrow} \cdots \longrightarrow \Lambda^{k} \mathbb{C}^{2 n} \stackrel{\sigma_{k}(\xi)}{\longrightarrow} \Lambda^{k+2} \mathbb{C}^{2 n} \\
& \longrightarrow \cdots \stackrel{\sigma_{2 n-2}(\xi)}{\longrightarrow} \Lambda^{2 n} \mathbb{C}^{2 n} \otimes \odot^{2 n-k-2} \mathbb{C}^{2} \longrightarrow 0
\end{aligned}
$$

is exact. Namely, $\operatorname{ker} \sigma_{0}(\xi)=\{0\}$,

$$
\operatorname{ker} \sigma_{j}(\xi)=\text { range } \sigma_{j-1}(\xi)
$$

for $j=1, \ldots, 2 n-2$, and $\sigma_{2 n-2}(\xi)$ is surjective.

Proof. Case 1. $j<k$. We need to show that

$$
\Lambda^{j-1} \mathbb{C}^{2 n} \otimes \odot \odot^{p+1} \mathbb{C}^{2} \stackrel{\sigma_{j-1}(\xi)}{\longrightarrow} \Lambda^{j} \mathbb{C}^{2 n} \otimes \odot \odot^{p} \mathbb{C}^{2} \stackrel{\sigma_{j}(\xi)}{\longrightarrow} \Lambda^{j+1} \mathbb{C}^{2 n} \otimes \odot^{p-1} \mathbb{C}^{2}
$$

is exact for $p=k-j$, where the linear mapping $\sigma_{j}(\xi)$ is given by

$$
\left[\sigma_{j}(\xi) \vartheta\right]_{A_{0} \ldots A_{j} A_{2}^{\prime} \ldots A_{p}^{\prime}}=\xi_{\left[A_{0}\right.}^{A^{\prime}} \vartheta_{\left.A_{1} \ldots A_{j}\right] A^{\prime} A_{2}^{\prime} \ldots A_{p}^{\prime}}=\frac{1}{j+1} \sum_{s=0}^{j}(-1)^{s} \xi_{A_{s}}^{A^{\prime}} \vartheta_{\ldots A_{0} \ldots A^{\prime} A_{2}^{\prime} \ldots A_{p}^{\prime}},
$$

for $\vartheta \in \Lambda^{j} \mathbb{C}^{2 n} \otimes \odot \odot^{p} \mathbb{C}^{2}$, by definitions of symbols and antisymmetrisation. Then

$$
\left[\sigma_{j}(\xi) \circ \sigma_{j-1}(\xi) \vartheta\right]_{A_{0} \ldots A_{j} A_{2}^{\prime} \ldots A_{p}^{\prime}}=\xi_{\left[\left[A_{0}\right.\right.}^{A^{\prime}} \xi_{\left.A_{1}\right]}^{B^{\prime}} \vartheta_{\left.\ldots A_{j}\right] B^{\prime} A^{\prime} A_{2}^{\prime} \ldots A_{p}^{\prime}}=0
$$

by $\xi_{\left[A_{0}\right.}^{\left(A^{\prime}\right.} \xi_{\left.A_{1}\right]}^{\left.B^{\prime}\right)}=0$ and $\vartheta$ symmetric in the primed indices. 
We can choose a transformation $M \in \mathrm{GL}(n, \mathbb{H})$ such that its complexification matrix $\tau(M) \in \mathrm{GL}(2 n, \mathbb{C})$ $\operatorname{transforming} \xi:=\left(\begin{array}{c}\xi_{00^{\prime}} \\ \vdots \\ \xi_{(2 n-1) 0^{\prime}}\end{array}\right) \neq 0$ to $\widetilde{\xi}:=\left(\begin{array}{c}1 \\ 0 \\ \vdots\end{array}\right)$. Note that $\left(\begin{array}{c}\xi_{01^{\prime}} \\ \vdots \\ \xi_{(2 n-1) 1^{\prime}}\end{array}\right)=-J\left(\begin{array}{c}\overline{\xi_{00^{\prime}}} \\ \vdots \\ \overline{\xi_{(2 n-1) 0^{\prime}}}\end{array}\right)$ by (3.23). It follows from $\tau(M) J=J \overline{\tau(M)}$ in (2.4) that

$$
\left(\widetilde{\xi}_{A A^{\prime}}\right)=\tau(M)\left(\xi_{A A^{\prime}}\right)=\left(\begin{array}{cc}
1 & 0 \\
0 & 1 \\
0 & 0 \\
\vdots & \vdots
\end{array}\right) .
$$

Then $\widetilde{\xi}_{A}^{A^{\prime}}=\tau(M)_{A}{ }^{B} \xi_{B}^{A^{\prime}}$ by raising indices. Set $\widetilde{\vartheta}_{A_{1} \ldots A_{j} A_{1}^{\prime} \ldots}:=\prod_{l=1}^{j} \tau(M)_{A_{l}}{ }^{B_{l}} \cdot \vartheta_{B_{1} \ldots B_{j} A_{1}^{\prime} \ldots}$. It is direct to see that

$$
\begin{aligned}
{\left[\sigma_{j}(\widetilde{\xi}) \widetilde{\vartheta}\right]_{A_{0} \ldots A_{j} A_{2}^{\prime} \ldots A_{p}^{\prime}} } & =\frac{1}{j+1} \sum_{s=0}^{j}(-1)^{s} \widetilde{\xi}_{A_{s}}^{A^{\prime}} \widetilde{\vartheta}_{A_{1} \ldots A_{0} \ldots A_{j} A^{\prime} A_{2}^{\prime} \ldots A_{p}^{\prime}} \\
& =\frac{1}{j+1} \sum_{s=0}^{j}(-1)^{s} \tau(M)_{A_{s}}{ }^{B_{s}} \xi_{B_{s}}^{A^{\prime}} \prod_{l \neq s} \tau(M)_{A_{l}}{ }^{B_{l}} \vartheta_{B_{1} \ldots B_{0} \ldots B_{j} A^{\prime} A_{2}^{\prime} \ldots A_{p}^{\prime}} \\
& =\prod_{l=0}^{j} \tau(M)_{A_{l}}{ }^{B_{l}} \cdot\left[\sigma_{j}(\xi) \vartheta\right]_{B_{0} \ldots B_{j} A^{\prime} A_{2}^{\prime} \ldots A_{p}^{\prime}}=\widetilde{\sigma \sigma(\xi) \vartheta}_{A_{0} \ldots A_{j} A_{2}^{\prime} \ldots A_{p}^{\prime}}
\end{aligned}
$$

So $\sigma_{j}(\xi) \vartheta=0$ if and only if $\sigma_{j}(\widetilde{\xi}) \widetilde{\vartheta}=0$. Suppose that the exactness is proved for $\widetilde{\xi}$ in (3.28). Then there exists $\widetilde{\kappa} \in \Lambda^{j-1} \mathbb{C}^{2 n} \otimes \odot^{p+1} \mathbb{C}^{2}$ such that $\sigma_{j-1}(\widetilde{\xi}) \widetilde{\kappa}=\widetilde{\vartheta}$. It follows that $\sigma_{j-1}(\xi) \kappa=\vartheta$ for $\kappa=\tau(M)^{-1} \widetilde{\kappa}$ by (3.29). So we only need to check the exactness of (3.26) for $\xi$ given by (3.28). By raising indices, we have

$$
\left(\xi_{A}^{A^{\prime}}\right)=\left(\begin{array}{cc}
0 & -1 \\
1 & 0 \\
0 & 0 \\
\vdots & \vdots
\end{array}\right)
$$

For $\vartheta \in \Lambda^{j} \mathbb{C}^{2 n} \otimes \odot{ }^{p} \mathbb{C}^{2}$, by definition (3.27) of $\sigma_{j}(\xi) \vartheta$ and $\xi_{A}^{A^{\prime}}$ in (3.30), we have (i)

$$
\left[\sigma_{j}(\xi) \vartheta\right]_{A_{0} \ldots A_{j} A_{2}^{\prime} \ldots A_{p}^{\prime}}=0, \quad \text { for } \quad 2 \leq A_{0}, \cdots, A_{j},
$$

since $\xi_{A_{j}}^{A^{\prime}}=0$ for such $A_{j}$ 's; (ii) for $2 \leq A_{1}, \cdots, A_{j}$,

$$
\left[\sigma_{j}(\xi) \vartheta\right]_{0 A_{1} \ldots A_{j} A_{2}^{\prime} \ldots A_{p}^{\prime}}=-\frac{1}{j+1} \vartheta_{A_{1} \ldots A_{j} 1^{\prime} A_{2}^{\prime} \ldots A_{p}^{\prime}}, \quad\left[\sigma_{j}(\xi) \vartheta\right]_{1 A_{1} \ldots A_{j} A_{2}^{\prime} \ldots A_{p}^{\prime}}=\frac{1}{j+1} \vartheta_{A_{1} \ldots A_{j} 0^{\prime} A_{2}^{\prime} \ldots A_{p}^{\prime}}
$$

(iii) for $2 \leq A_{2}, \cdots, A_{j}$,

$$
\left[\sigma_{j}(\xi) \vartheta\right]_{01 A_{2} \ldots A_{j} A_{2}^{\prime} \ldots A_{p}^{\prime}}=-\frac{1}{j+1}\left(\vartheta_{1 A_{2} \ldots A_{j} 1^{\prime} A_{2}^{\prime} \ldots A_{p}^{\prime}}+\vartheta_{0 A_{2} \ldots A_{j} 0^{\prime} A_{2}^{\prime} \ldots A_{p}^{\prime}}\right) .
$$

Therefore $\vartheta \in \operatorname{ker} \sigma_{j}(\xi)$ for $j \geq 1$ if and only if

$$
\vartheta_{A_{1} \ldots A_{j} A_{1}^{\prime} A_{2}^{\prime} \ldots A_{p}^{\prime}}=0, \quad \vartheta_{1 A_{2} \ldots A_{j} 1^{\prime} A_{2}^{\prime} \ldots A_{p}^{\prime}}=-\vartheta_{0 A_{2} \ldots A_{j} 0^{\prime} A_{2}^{\prime} \ldots A_{p}^{\prime}},
$$

for any $A_{1}^{\prime}, A_{2}^{\prime}, \ldots, A_{p}^{\prime}=0^{\prime}, 1^{\prime}$ and $2 \leq A_{1}<\cdots<A_{j}$. While for $j=0, \vartheta \in \operatorname{ker} \sigma_{0}(\xi)$ if and only if $\vartheta_{A_{1}^{\prime} A_{2}^{\prime} \ldots A_{p}^{\prime}}=0$. So $\vartheta \in \operatorname{ker} \sigma_{0}(\xi)=\{0\}$. 
To show the surjectivity of $\sigma_{j-1}(\xi)$, we need to find an element $\Theta \in \Lambda^{j-1} \mathbb{C}^{2 n} \otimes \odot^{p+1} \mathbb{C}^{2}$ such that $\sigma_{j-1}(\xi) \Theta=\vartheta$ for $\vartheta$ satisfying (3.34). We define such an element $\Theta$ by

$$
\begin{aligned}
& \Theta_{A_{2} \ldots A_{j} 0^{\prime} 1^{\prime} A_{2}^{\prime} \ldots A_{p}^{\prime}}:=j \vartheta_{1 A_{2} \ldots A_{j} 1^{\prime} A_{2}^{\prime} \ldots A_{p}^{\prime}}=-j \vartheta_{0 A_{2} \ldots A_{j} 0^{\prime} A_{2}^{\prime} \ldots A_{p}^{\prime},}, \quad \Theta_{A_{2} \ldots A_{j} 0^{\prime} 0^{\prime} 0^{\prime} \ldots 0^{\prime}}=j \theta_{0 A_{2} \ldots A_{j} 0^{\prime} 0^{\prime} \ldots 0^{\prime}},
\end{aligned}
$$

for $2 \leq A_{2}, \cdots, A_{j}$, and

$$
\Theta_{1 A_{3} \ldots A_{j} 1^{\prime} A_{1}^{\prime} \ldots A_{p}^{\prime}}+\Theta_{0 A_{3} \ldots A_{j} 0^{\prime} A_{1}^{\prime} \ldots A_{p}^{\prime}}=-j \vartheta_{01 A_{3} \ldots A_{j} A_{1}^{\prime} A_{2}^{\prime} \ldots A_{p}^{\prime}}
$$

and all other kind of entries vanish. Obviously, there exists an element $\Theta$ satisfying conditions (3.35)(3.36), but such $\Theta$ is not unique. Then by definition (3.27) for the symbol $\sigma_{j-1}(\xi)$ again, as in (3.31)(3.33), it is easy to see that for any $2 \leq A_{1}, \cdots, A_{j}$, we have $\left[\sigma_{j-1}(\xi) \Theta\right]_{A_{1} \ldots A_{j} A_{1}^{\prime} A_{2}^{\prime} \ldots A_{p}^{\prime}}=0$, and

$$
\begin{aligned}
{\left[\sigma_{j-1}(\xi) \Theta\right]_{0 A_{2} \ldots A_{j} 0^{\prime} A_{2}^{\prime} \ldots A_{p}^{\prime}} } & =-\frac{1}{j} \Theta_{A_{2} \ldots A_{j} 1^{\prime} 0^{\prime} A_{2}^{\prime} \ldots A_{p}^{\prime}}=\vartheta_{0 A_{2} \ldots A_{j} 0^{\prime} A_{2}^{\prime} \ldots A_{p}^{\prime},} \\
{\left[\sigma_{j-1}(\xi) \Theta\right]_{1 A_{2} \ldots A_{j} 1^{\prime} A_{2}^{\prime} \ldots A_{p}^{\prime}} } & =\frac{1}{j} \Theta_{A_{2} \ldots A_{j} 0^{\prime} 1^{\prime} A_{2}^{\prime} \ldots A_{p}^{\prime}}=\vartheta_{1 A_{2} \ldots A_{j} 1^{\prime} A_{2}^{\prime} \ldots A_{p}^{\prime}},
\end{aligned}
$$

by (3.35), and

$$
\begin{aligned}
& {\left[\sigma_{j-1}(\xi) \Theta\right]_{0 A_{2} \ldots A_{j} 1^{\prime} 1^{\prime} \ldots 1^{\prime}}=-\frac{1}{j} \Theta_{A_{2} \ldots A_{j} 1^{\prime} 1^{\prime} 1^{\prime} \ldots 1^{\prime}}=\theta_{0 A_{2} \ldots A_{j} 1^{\prime} 1^{\prime} \ldots 1^{\prime}},} \\
& {\left[\sigma_{j-1}(\xi) \Theta\right]_{1 A_{2} \ldots A_{j} 0^{\prime} 0^{\prime} \ldots 0^{\prime}}=\frac{1}{j} \Theta_{A_{2} \ldots A_{j} 0^{\prime} 0^{\prime} 0^{\prime} \ldots 0^{\prime}}=\theta_{0 A_{2} \ldots A_{j} 0^{\prime} 0^{\prime} \ldots 0^{\prime}}}
\end{aligned}
$$

by (3.35) again, and

$$
\left[\sigma_{j-1}(\xi) \Theta\right]_{01 A_{3} \ldots A_{j} A_{1}^{\prime} \ldots A_{p}^{\prime}}=-\frac{1}{j}\left(\Theta_{1 A_{3} \ldots A_{j} 1^{\prime} A_{1}^{\prime} \ldots A_{p}^{\prime}}+\Theta_{0 A_{3} \ldots A_{j} 0^{\prime} A_{1}^{\prime} \ldots A_{p}^{\prime}}\right)=\vartheta_{01 A_{3} \ldots A_{j} A_{1}^{\prime} A_{2}^{\prime} \ldots A_{p}^{\prime},}
$$

by (3.36). Thus $\sigma_{j-1}(\xi) \Theta=\vartheta$, and so the sequence (3.26) is exact.

Case 2. $j=k$. We need to show that

$$
\Lambda^{k-1} \mathbb{C}^{2 n} \otimes \mathbb{C}^{2} \stackrel{\sigma_{k-1}(\xi)}{\longrightarrow} \Lambda^{k} \mathbb{C}^{2 n} \stackrel{\sigma_{k}(\xi)}{\longrightarrow} \Lambda^{k+2} \mathbb{C}^{2 n}
$$

is exact. For $\vartheta \in \Lambda^{k} \mathbb{C}^{2 n}$, we have

$$
\left[\sigma_{k}(\xi) \vartheta\right]_{A_{1} \ldots A_{k+2}}=\xi_{A^{\prime}\left[A_{1}\right.} \xi_{A_{2}}^{A^{\prime}} \vartheta_{\left.A_{3} \ldots A_{k+2}\right]} .
$$

$\sigma_{k}(\xi) \circ \sigma_{k-1}(\xi)=0$ holds as before. Without loss of generality, we can assume $\xi$ is given by (3.28) as in case 1 . We only need to consider unprimed indices with $A_{1}<\cdots<A_{k+2}$. Then for this $\xi$, we have

$$
\left[\sigma_{k}(\xi) \vartheta\right]_{A_{1} \ldots A_{k+2}}= \begin{cases}\frac{2}{(k+2)(k+1)} \vartheta_{A_{3} \ldots A_{k+2}}, & \text { if } A_{1}=0, A_{2}=1, \\ 0, & \text { otherwise }\end{cases}
$$

by $\xi_{A^{\prime}\left[A_{1}\right.} \xi_{\left.A_{2}\right]}^{A^{\prime}}=0$ except for $\xi_{A^{\prime}[0} \xi_{1]}^{A^{\prime}}=1$. Therefore $\operatorname{ker} \sigma_{k}(\xi)$ consists of $\vartheta \in \Lambda^{k} \mathbb{C}^{2 n}$ with $\vartheta_{B_{1} \ldots B_{k}}=0$ for $2 \leq B_{1}, \cdots, B_{k}$. On the other hand, for $\Theta \in \Lambda^{k-1} \mathbb{C}^{2 n} \otimes \mathbb{C}^{2}$ and $2 \leq A_{1}, \cdots, A_{k}$, we have

$$
\begin{aligned}
& {\left[\sigma_{k-1}(\xi) \Theta\right]_{A_{1} A_{2} \ldots A_{k}}=0,} \\
& {\left[\sigma_{k-1}(\xi) \Theta\right]_{0 A_{2} \ldots A_{k}}=-\frac{1}{k} \Theta_{A_{2} \ldots A_{k} 1^{\prime}}, \quad\left[\sigma_{k-1}(\xi) \Theta\right]_{1 A_{2} \ldots A_{k}}=\frac{1}{k} \Theta_{A_{2} \ldots A_{k} 0^{\prime}},} \\
& {\left[\sigma_{k-1}(\xi) \Theta\right]_{01 A_{3} \ldots A_{k}}=-\frac{1}{k}\left(\Theta_{1 A_{3} \ldots A_{k} 1^{\prime}}+\Theta_{0 A_{3} \ldots A_{k} 0^{\prime}}\right) .}
\end{aligned}
$$


Hence as in the case 1 , if we set $\Theta_{A_{2} \ldots A_{k} 1^{\prime}}:=-k \vartheta_{0 A_{2} \ldots A_{k}}, \Theta_{A_{2} \ldots A_{k} 0^{\prime}}:=k \vartheta_{1 A_{2} \ldots A_{k}}$ and

$$
\Theta_{1 A_{3} \ldots A_{k} 1^{\prime}}+\Theta_{0 A_{3} \ldots A_{k} 0^{\prime}}=-k \vartheta_{01 A_{3} \ldots A_{k}}
$$

for $A_{2}, \cdots, A_{k} \geq 2$, and all other kind of entries vanish, then we get $\sigma_{k-1}(\xi) \Theta=\vartheta$. So (3.37) is exact.

Case 3. $j \geq k+1$. It is similar. We omit details.

We can consider the associated Laplacian operators as in the flat case [45, some of which are of order 4. This is because $D_{k}^{(k)}$ 's are of order 2. They are all self adjoint elliptic operators. Then by applying the standard theory of elliptic operators [49, we can obtain the following theorem.

Theorem 3.2. Suppose that $M$ is a compact unimodular quaternionic manifold and is right conformally flat if $\operatorname{dim}_{\mathbb{R}} M=4$. Then we have the Hodge-type orthogonal decomposition:

$$
\Gamma\left(\mathscr{V}_{j}^{(k)}\right)=\operatorname{Image} D_{j-1}^{(k)} \oplus \operatorname{Image} D_{j}^{(k) *} \oplus \mathscr{H}_{(k)}^{j}(M),
$$

where $\mathscr{H}_{(k)}^{j}(M) \cong\left\{f \in \Gamma\left(\mathscr{V}_{j}^{(k)}\right) ; D_{j}^{(k)} f=0, D_{j-1}^{(k) *} f=0\right\} \cong H_{(k)}^{j}(M)$ are finite dimensional.

See the Appendix for a detailed proof. For domains in $\mathbb{H}^{n}$, the cohomology groups of the $k$-CauchyFueter complex are much more difficult to study. In [15] [48, we got results for the associated Neumann problem over domains in $\mathbb{H}$.

\section{VANishing TheOREM OVER QUATERnionic KähleR MANifoldS}

We only consider quaternionic Kähler manifolds in this section.

Theorem 4.1. Suppose that $M$ is a compact quaternionic Kähler manifold (right conformally flat if $\left.\operatorname{dim}_{\mathbb{R}} M=4\right)$ with negative scalar curvature. Then we have $H_{(k)}^{j}(M)=\{0\}$ for $j=1, \ldots, k-1$.

Horan 22] 23. proved the Weitzenböck formula and the vanishing theorem for the first cohomology group of Salamon's complex. See also theorem 4.3 of Nagatomo-Nitta 26] for vanishing theorem for Salamon's complexes when the manifolds have negative scalar curvatures, which essentially implies the vanishing of $H_{(k)}^{j}(M)$ of the $k$-Cauchy-Fueter complex for $j \geq k+3$. See also [37] for indices of Salamon's complexes.

It is sufficient to prove the associated Weitzenböck formula. In this section, on a quaternionic Kähler manifold, we will choose coordinate charts $U_{\alpha}$ with trivialization $\left.E^{*}\right|_{U_{\alpha}}=U_{\alpha} \times \mathbb{C}^{2 n},\left.H^{*}\right|_{U_{\alpha}}=U \times \mathbb{C}^{2}$, two-component local orthonormal quaternionic frame $\left\{Z_{A A^{\prime}}\right\}$ (2.8) such that $g, \varepsilon$ and $\epsilon$ are standard:

$$
\left(\epsilon_{A B}\right)=\left(\begin{array}{rrrrr}
0 & 1 & & & \\
-1 & 0 & & & \\
& & \ddots & & \\
& & & 0 & 1 \\
& & & -1 & 0
\end{array}\right) .
$$

$\left(\epsilon^{A B}\right)$ is the inverse of $\left(\epsilon_{A B}\right)$. They are used to raise or lower unprimed indices, e.g. $\nabla_{A^{\prime}}^{A}=\nabla_{A^{\prime} B} \epsilon^{B A}$. Define inner product locally as

$$
\langle v, w\rangle:=\sum_{B_{1}, \ldots, B_{p}^{\prime}} v_{B_{1} \ldots B_{q} B_{1}^{\prime} \ldots B_{p}^{\prime}} \overline{w_{B_{1} \ldots B_{q} B_{1}^{\prime} \ldots B_{p}^{\prime}}}
$$


for two $\mathfrak{T}_{q, p}$-tensor $v$ and $w$, and $|v|^{2}:=\langle v, v\rangle$. It is obviously well defined globally since it is invariant under the transformation $v \rightarrow \widetilde{v}(w \rightarrow \widetilde{w})$ with

$$
\widetilde{v}_{A_{1} \ldots A_{q} A_{1}^{\prime} \ldots A_{p}^{\prime}}=\prod_{j=1}^{q} \Phi_{A_{j}}{ }^{B_{j}} \prod_{l=1}^{p} \Psi_{A_{l}^{\prime}}^{B_{l}^{\prime}} v_{B_{1} \ldots B_{q} B_{1}^{\prime} \ldots B_{p}^{\prime}},
$$

for $\Phi \in \mathrm{SU}(2 n), \Psi \in \mathrm{SU}(2)$. It is an Hermitian inner product. The covariant derivatives can be extended naturally to $\left(\otimes^{r} E\right) \otimes\left(\otimes^{s} H\right) \otimes\left(\otimes^{q} E^{*}\right) \otimes\left(\otimes^{p} H^{*}\right)$. Define $L^{2}$-inner product $(f, h):=\int_{M}\langle f, h\rangle d V_{g}$, where $d V_{g}$ is the volume form (2.9) of the metric $g$. Denote $\|f\|:=(f, f)^{\frac{1}{2}}$.

Theorem 4.1 is a consequence of the Hodge-type decomposition in Theorem 3.2 and the following Weitzenböck formula, because the right hand side of (4.2) is negative if the scalar curvature is negative.

Proposition 4.1. Suppose that $f \in \Gamma\left(\Lambda^{j} E^{*} \otimes \odot^{k-j} H^{*}\right)$ satisfying $D_{j}^{(k)} f=0, D_{j-1}^{(k) *} f=0$ on a compact quaternionic Kähler manifold $M$ of dimension $4 n$ (it is right conformally flat if $\operatorname{dim}_{\mathbb{R}} M=4$ ), $j=$ $1, \ldots, k-1$. Then we have

$$
\|\widehat{\nabla} f\|^{2}+c_{j}\left\|\nabla^{*} f\right\|^{2}=\frac{2 n-1+(-1)^{j}}{(j+1)}(p+2) \int_{M} \Lambda|f|^{2} d V_{g},
$$

where $p=k-j, c_{j}=\frac{1-(-1)^{j}}{2(j+1)} \frac{p+2}{p+1} \geq 0, \Lambda=\frac{s_{g}}{8 n(n+2)}$, and $s_{g}$ is the scalar curvature.

Remark 4.1. On quaternionic Kähler manifold, Semmelmann and Weingart 34 derived Weitzenböck formulae only involving twisted Dirac and twistor operators, but not for our operators $D_{j}^{(k)}$ 's. They [35] obtained universal Weitzenböck formula for all irreducible non-symmetric holonomy groups, and an recursive procedure for obtaining coefficients of Weitzenböck formulas for the holonomy groups $\mathrm{SO}(n)$, $G_{2}$ and $\operatorname{Spin}(7)$. But the recursive formula for coefficients of Weitzenböck formulae for the holonomy group $\mathrm{Sp}(n) \mathrm{Sp}(1)$ was not given there. Even if we obtain the recursive formula and know the concrete Weitzenböck formulae, we also need combine several Weitzenböck formulae to obtain the identity (4.2). It will be a tedious algebraic calculation to derive (4.2) from universal Weitzenböck formula in [35. See also Homma 21] for Weitzenböck formulae over quaternionic Kähler manifolds.

4.1. The formal adjoint operators. By two-component notation, we can derive the formal adjoint operators of $\nabla$ and $\mathscr{D}_{q, p}$ explicitly, while there is no proof for them in Horan [22] [23].

Proposition 4.2. On a quaternionic Kähler manifold, we have

$$
Z^{A A^{\prime}}=\overline{Z_{A A^{\prime}}}
$$

and

$$
\overline{\Gamma_{A A^{\prime} B}{ }^{C}}=-\Gamma_{C}^{A A^{\prime}}{ }_{C}^{B}, \quad \overline{\Gamma_{A A^{\prime} B^{\prime}} C^{\prime}}=-\Gamma_{C^{\prime}}^{A A^{\prime}} .
$$

Moreover, the formal adjoint of $Z_{A A^{\prime}}$ is

$$
Z_{A A^{\prime}}^{*}=-Z^{A A^{\prime}}+\Gamma_{D}^{D A^{\prime}}{ }^{A}+\Gamma_{D^{\prime}}^{A D^{\prime}}{ }^{A^{\prime}} .
$$

Proof. (1) By definition, $Z^{A A^{\prime}}:=Z_{B B^{\prime}} \epsilon^{B A} \varepsilon^{B^{\prime} A^{\prime}}$ and $\epsilon^{10}=-\epsilon^{01}=1, \varepsilon^{1^{\prime} 0^{\prime}}=-\varepsilon^{0^{\prime} 1^{\prime}}=1$. It is direct from definition of $Z_{A A^{\prime}}$ 's in (2.8) to see that

$$
\begin{array}{ll}
\overline{Z_{00^{\prime}}}=Z_{11^{\prime}}=Z^{00^{\prime}}, & \overline{Z_{10^{\prime}}}=-Z_{01^{\prime}}=Z^{10^{\prime}}, \\
\overline{Z_{01^{\prime}}}=-Z_{10^{\prime}}=Z^{01^{\prime}}, & \overline{Z_{11^{\prime}}}=Z_{00^{\prime}}=Z^{11^{\prime}}, \cdots .
\end{array}
$$


(2) On a quaternionic Kähler manifold $M$, we know that for any $X \in T M$, the connection $\omega^{\prime}(X)$ on the bundle $E$ is $\mathfrak{s u}(2 n)$-valued. If write $Z_{A A^{\prime}}=X+\mathbf{i} Y$ for some $X, Y \in T M$, we have

$$
\overline{\Gamma_{A A^{\prime} B}{ }^{C}}=\overline{\omega^{\prime}(X+\mathbf{i} Y)_{B}{ }^{C}}=\overline{\omega^{\prime}(X)_{B}{ }^{C}}-\overline{\mathbf{i} \omega^{\prime}(Y)_{B}{ }^{C}}=-\omega^{\prime}\left(\overline{\left.Z_{A A^{\prime}}\right)_{C}{ }^{B}}=-\Gamma^{A A^{\prime}}{ }_{C}{ }^{B} .\right.
$$

Here we have used $\overline{Z_{A A^{\prime}}}=Z^{A A^{\prime}}$ in (4.3). It is similar for $\Gamma_{A A^{\prime} B^{\prime}}{ }^{C^{\prime}}$.

(3) Recall that the formal adjoint $Z_{A A^{\prime}}^{*}$ of $Z_{A A^{\prime}}$ satisfies

$$
\int_{M} Z_{A A^{\prime}} f \cdot \bar{h} d V_{g}=\int_{M} f \cdot \overline{Z_{A A^{\prime}}^{*}} d V_{g}
$$

for any compactly supported scalar functions $f$ and $h$. Note that $X f=i_{X} d f$, where $i_{X}$ is the interior operator. By Stokes' formula, we have

$$
\int_{M} Z_{A A^{\prime}}(f \bar{h}) d V_{g}=\int_{M} d(f \bar{h}) \wedge i_{Z_{A A^{\prime}}} d V_{g}=-\int_{M} f \bar{h} \cdot d\left(i_{Z_{A A^{\prime}}} d V_{g}\right),
$$

where the volume form $d V_{g}$ is given by (2.9), $\left\{e^{B B^{\prime}}\right\}$ is dual to $\left\{Z_{B B^{\prime}}\right\}$ and

$$
d\left(i_{Z_{A A^{\prime}}} d V_{g}\right)=\sum_{\left(B, B^{\prime}\right) \neq\left(A, A^{\prime}\right)} d e^{B B^{\prime}} \wedge i_{Z_{B B^{\prime}}} i_{Z_{A A^{\prime}}} d V_{g} .
$$

By the standard exterior differentiation formula, $d \varphi(X, Y)=\frac{1}{2}\left[\left(\nabla_{X} \varphi\right)(Y)-\left(\nabla_{Y} \varphi\right)(X)+\varphi\left(\tau_{X, Y}\right)\right]$ for 1-form $\varphi \in \Omega^{1}(M)$ and the torsion $\tau_{X, Y}=0$ since the connection is torsion-free, we find that

$$
\begin{aligned}
2 d e^{B B^{\prime}}\left(Z_{C C^{\prime}}, Z_{D D^{\prime}}\right) & =\left(-\Gamma_{C C^{\prime} E}^{B} e^{E B^{\prime}}-\Gamma_{C C^{\prime} E^{\prime}}^{B^{\prime}} e^{B E^{\prime}}\right)\left(Z_{D D^{\prime}}\right)-C C^{\prime} \leftrightarrow D D^{\prime} \\
& =\left(-\Gamma_{C C^{\prime} D^{B}} \delta_{D^{\prime}}^{B^{\prime}}-\Gamma_{C C^{\prime} D^{\prime}} B^{B^{\prime}} \delta_{D}^{B}\right)-C C^{\prime} \leftrightarrow D D^{\prime} .
\end{aligned}
$$

Using $\omega_{1} \wedge \omega_{2}(X, Y):=\frac{1}{2}\left[\omega_{1}(X) \omega_{2}(Y)-\omega_{1}(Y) \omega_{2}(X)\right]$, we get the Cartan formula

$$
d e^{B B^{\prime}}=-\left(\Gamma_{C C^{\prime} D}^{B} \delta_{D^{\prime}}^{B^{\prime}}+\Gamma_{C C^{\prime} D^{\prime}}^{B^{\prime}} \delta_{D}^{B}\right) e^{C C^{\prime}} \wedge e^{D D^{\prime}} .
$$

Note that for fixed $A A^{\prime}, B B^{\prime}$, we can write $d V_{g}=e^{A A^{\prime}} \wedge e^{B B^{\prime}} \wedge \widetilde{\omega}$ with the (4n-2)-form $\widetilde{\omega}=i_{Z_{B B^{\prime}}} i_{Z_{A A^{\prime}}} d V_{g}$. Substitute it and (4.9) into (4.8) to get

$$
d\left(i_{Z_{A A^{\prime}}} d V_{g}\right)=-\sum_{\left(B, B^{\prime}\right) \neq\left(A, A^{\prime}\right)}\left(\Gamma_{A A^{\prime} B}{ }^{B}+\Gamma_{A A^{\prime} B^{\prime}}^{B^{\prime}}-\Gamma_{B B^{\prime} A}{ }^{B} \delta_{A^{\prime}}^{B^{\prime}}-\Gamma_{B B^{\prime} A^{\prime}}{ }^{B^{\prime}} \delta_{A}^{B}\right) d V_{g}
$$

Now substituting the conjugate of (4.10) into the right hand side of (4.7), we find that for $A$ and $A^{\prime}$ fixed,

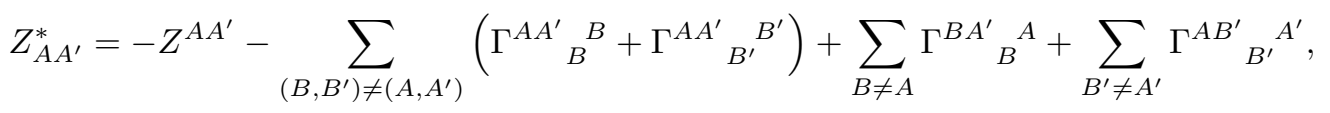

by using definition (4.6) and $\overline{Z_{A A^{\prime}}}=Z^{A A^{\prime}}$ in (4.3). But for $A$ and $A^{\prime}$ fixed, we have

$$
\sum_{\left(B, B^{\prime}\right) \neq\left(A, A^{\prime}\right)} \Gamma_{B}^{A A^{\prime}{ }^{B}}=\Gamma_{A}^{A A^{\prime}}{ }_{A}^{A}+2 \sum_{B \neq A} \Gamma_{B}^{A A^{\prime}{ }_{B}^{B}}=-\Gamma_{A}^{A A^{\prime}}{ }_{A}^{A}
$$

by $\operatorname{tr} \Gamma_{a *}{ }^{*}=0$ since it is also $\mathfrak{s l}(2 n, \mathbb{C})$-valued, and similarly

$$
\sum_{\left(B, B^{\prime}\right) \neq\left(A, A^{\prime}\right)} \Gamma_{B^{\prime}}^{A A^{\prime}} B^{B^{\prime}}=\sum_{B^{\prime} \neq A^{\prime}} \Gamma_{B^{\prime}}^{A A^{\prime}} B^{B^{\prime}}+(2 n-1) \sum_{B^{\prime}} \Gamma_{B^{\prime}}^{A A^{\prime}}{ }_{B^{\prime}}=-\Gamma_{A^{\prime}}^{A A^{\prime}},
$$

by $\operatorname{tr} \Gamma_{a *^{\prime}} *^{\prime}=0$. Now (4.5) follows from substituting (4.12)-(4.13) into (4.11). 
Proposition 4.3. On a quaternionic Kähler manifold, we have

(1) The formal adjoint $\nabla^{*}: \Gamma\left(\mathfrak{T}_{q+1, p+1}\right) \rightarrow \Gamma\left(\mathfrak{T}_{q, p}\right)$ of $\nabla$ is given by

$$
\left(\nabla^{*} f\right)_{A_{1} \ldots A_{q} A_{1}^{\prime} \ldots A_{p}^{\prime}}=-\nabla^{A A^{\prime}} f_{A A_{1} \ldots A_{q} A^{\prime} A_{1}^{\prime} \ldots A_{p}^{\prime}}
$$

(2) the formal adjoint $\mathscr{D}_{q, p}^{*}: \Gamma\left(\Lambda^{q+1} E^{*} \otimes \odot^{p-1} H^{*}\right) \longrightarrow \Gamma\left(\Lambda^{q} E^{*} \otimes \odot^{p} H^{*}\right)$ of $\mathscr{D}_{q, p}$ is given by

$$
\left(\mathscr{D}_{q, p}^{*} f\right)_{A_{1} \ldots A_{q} A_{1}^{\prime} \ldots A_{p}^{\prime}}=\nabla_{\left(A_{1}^{\prime}\right.}^{A} f_{\left.\left|A A_{1} \ldots A_{q}\right| A_{2}^{\prime} \ldots A_{p}^{\prime}\right)} .
$$

Proof. (1) For any local section $h \in \Gamma\left(\mathfrak{T}_{q, p}\right)$, we have

$$
\begin{aligned}
& (\nabla h, f)=\int \nabla_{B B^{\prime}} h_{A_{1} \ldots A_{q} A_{1}^{\prime} \ldots A_{p}^{\prime}} \overline{f_{B A_{1} \ldots A_{q} B^{\prime} A_{1}^{\prime} \ldots A_{p}^{\prime}}} d V_{g} \\
& =\int\left[Z_{B B^{\prime}} h_{A_{1} \ldots A_{q} A_{1}^{\prime} \ldots A_{p}^{\prime}}-\Gamma_{B B^{\prime} A_{j}}^{{ }^{D}} h_{A_{1} \ldots D \ldots A_{q} A_{1}^{\prime} \ldots A_{p}^{\prime}}\right. \\
& \left.-\Gamma_{B B^{\prime} A_{j}^{\prime}}^{D^{\prime}} h_{A_{1} \ldots A_{q} A_{1}^{\prime} \ldots D^{\prime} \ldots A_{p}^{\prime}}\right] \overline{f_{B A_{1} \ldots A_{q} B^{\prime} A_{1}^{\prime} \ldots A_{p}^{\prime}}} d V_{g} \\
& =\int h_{A_{1} \ldots A_{q} A_{1}^{\prime} \ldots A_{p}^{\prime}}\left[-\overline{Z^{B B^{\prime}}}+\overline{\Gamma_{D}^{D B^{\prime}{ }^{B}}}+\overline{\Gamma_{D^{\prime}}^{B D^{\prime}}{ }^{B^{\prime}}}\right] \overline{f_{B A_{1} \ldots A_{q} B^{\prime} A_{1}^{\prime} \ldots A_{p}^{\prime}}} \\
& +\left[h_{A_{1} \ldots D \ldots A_{q} A_{1}^{\prime} \ldots A_{p}^{\prime}} \overline{\Gamma_{D}^{B B^{\prime}} A_{j}}+h_{A_{1} \ldots A_{q} A_{1}^{\prime} \ldots D^{\prime} \ldots A_{p}^{\prime}} \overline{\Gamma_{D^{\prime}}^{B B^{\prime}} A_{j}^{\prime}}\right] \overline{f_{B A_{1} \ldots A_{q} B^{\prime} A_{1}^{\prime} \ldots A_{p}^{\prime}}},
\end{aligned}
$$

by using (4.5) for the expression of the formal adjoint $Z_{B B^{\prime}}^{*}$ and (4.4) for the connection coefficients. Then we can relabel indices so that the right hand side can be written as $\left(h, \nabla^{*} f\right)$ with

$$
\left(\nabla^{*} f\right)_{A_{1} \ldots A_{q} A_{1}^{\prime} \ldots A_{p}^{\prime}}=-Z^{A_{0} A_{0}^{\prime}} f_{A_{0} A_{1} \ldots A_{q} A_{0}^{\prime} A_{1}^{\prime} \ldots A_{p}^{\prime}}+\Gamma_{A_{j}}^{A_{0} A_{0}^{\prime}}{ }^{E} \ldots E \ldots A_{0}^{\prime} \ldots A_{p}^{\prime}+\Gamma_{A_{j}^{\prime}}^{A_{0} A_{0}^{\prime}} f_{A_{0} A_{1} \ldots A_{q} \ldots E^{\prime} \ldots} .
$$

(2) For any local sections $h \in \Gamma\left(\Lambda^{q} E^{*} \otimes \odot^{p} H^{*}\right)$ and $f \in \Gamma\left(\Lambda^{q+1} E^{*} \otimes \odot^{p-1} H^{*}\right)$, we have

$$
\begin{aligned}
& \left(\mathscr{D}_{q, p} h, f\right)=\int \nabla_{\left[A^{\prime}\right.}^{A^{\prime}} h_{\left.A_{1} \ldots A_{q}\right] A^{\prime} A_{2}^{\prime} \ldots A_{p}^{\prime}} \overline{f_{A A_{1} \ldots A_{q} A_{2}^{\prime} \ldots A_{p}^{\prime}}} d V_{g} \\
& =-\int \nabla_{A B^{\prime}} h_{A_{1} \ldots A_{q} A^{\prime} A_{2}^{\prime} \ldots A_{p}^{\prime}} \varepsilon_{B^{\prime} A^{\prime}} \cdot \overline{f_{A A_{1} \ldots A_{q} A_{2}^{\prime} \ldots A_{p}^{\prime}}} d V_{g} \\
& =\int h_{A_{1} \ldots A_{q} A^{\prime} A_{2}^{\prime} \ldots A_{p}^{\prime}} \overline{\nabla B^{\prime} f_{A A_{1} \ldots A_{q} A_{2}^{\prime} \ldots A_{p}^{\prime} \varepsilon_{B^{\prime} A^{\prime}}}} d V_{g} \\
& =\int h_{A_{1} \ldots A_{q} A^{\prime} A_{2}^{\prime} \ldots A_{p}^{\prime}} \overline{\nabla_{\left(A^{\prime}\right.}^{A} f_{\left.\left|A A_{1} \ldots A_{q}\right| A_{2}^{\prime} \ldots A_{p}^{\prime}\right)}} d V_{g}=\left(h, \mathscr{D}_{q, p}^{*} f\right)
\end{aligned}
$$

by using (1) and $\varepsilon^{B^{\prime} A^{\prime}}=-\varepsilon_{B^{\prime} A^{\prime}}$. Here we drop antisymmetrisation in the second identity by

$$
\sum_{B_{0}, \ldots, B_{q}}\left(g_{\left[B_{0} \ldots B_{q}\right] \mathscr{B}^{\prime}}, \widetilde{g}_{\left[B_{0} \ldots B_{q}\right] \mathscr{B}^{\prime}}\right)=\sum_{B_{0}, \ldots, B_{q}}\left(g_{B_{0} \ldots B_{q} \mathscr{B}^{\prime}}, \widetilde{g}_{\left[B_{0} \ldots B_{q}\right] \mathscr{B}^{\prime}}\right)
$$

for any local sections $g, \widetilde{g} \in \Gamma\left(\otimes^{q+1} E^{*} \otimes\left(\otimes^{p} H^{*}\right)\right)$, and add symmetrisation in the last identity by

$$
\sum_{B_{1}^{\prime}, \ldots, B_{p}^{\prime}}\left(h_{\mathscr{B}\left(B_{1}^{\prime} \ldots B_{p}^{\prime}\right)}, \widetilde{h}_{\mathscr{B} B_{1}^{\prime} \ldots B_{p}^{\prime}}\right)=\sum_{B_{1}^{\prime}, \ldots, B_{p}^{\prime}}\left(h_{\mathscr{B}\left(B_{1}^{\prime} \ldots B_{p}^{\prime}\right)}, \widetilde{h}_{\mathscr{B}\left(B_{1}^{\prime} \ldots B_{p}^{\prime}\right)}\right)
$$

for any local sections $h, \widetilde{h} \in \Gamma\left(\otimes^{q} E^{*} \otimes\left(\otimes^{p} H^{*}\right)\right)$. (4.14)-4.15) follows from definition easily. See (3.4) in 47] for the proof of (4.15). 
4.2. A Weitzenböck formula. The Weitzenböck formula for the $k$-Cauchy-Fueter complex is much more complicated than that for the De Rham complex, since we have not only exterior forms, but also symmetric forms. Note that $D_{j-1}^{(k) *} f=0$, i.e. $\mathscr{D}_{q-1, p+1}^{*} f=0$ for $q=j, p=k-j$, implies that

$$
\nabla_{\left(B_{1}^{\prime}\right.}^{A} f_{\left.\left|A B_{1} \ldots B_{q-1}\right| B_{2}^{\prime} \ldots B_{p+1}^{\prime}\right)}=0 .
$$

by Proposition 4.3 (2). The following is another version of lemma 2.1 of Horan 22 without proof.

Lemma 4.1. For a tensor $f \in \Gamma\left(\mathfrak{T}_{q, r}\right)$ such that

$$
f_{\mathscr{A} A} A_{1}^{\prime} A_{2}^{\prime} \ldots A_{r}^{\prime}=f_{\mathscr{A} A}^{\prime}\left(A_{2}^{\prime} \ldots A_{r}^{\prime}\right) \quad \text { and } \quad f_{\mathscr{A}\left(A_{1}^{\prime} A_{2}^{\prime} \ldots A_{r}^{\prime}\right)}=0
$$

we have

$$
f_{\mathscr{A} A_{1}^{\prime} A_{2}^{\prime} \ldots A_{r}^{\prime}}=-\frac{r-1}{r} \varepsilon_{A_{1}^{\prime}\left(A_{2}^{\prime}\right.} f_{|\mathscr{A}|} C^{C^{\prime}}{ }_{\left.A_{3}^{\prime} \ldots A_{r}^{\prime}\right) C^{\prime}} .
$$

Proof. Suppose that $A_{1}^{\prime}+\cdots+A_{r}^{\prime}=l$, i.e. there are $l$ 's $1^{\prime}$ in $\left\{A_{1}^{\prime}, \ldots, A_{r}^{\prime}\right\}$. Note that (4.17) implies that $f_{\mathscr{A} A_{1}^{\prime}\left(A_{2}^{\prime} \ldots A_{r}^{\prime}\right)}+\cdots+f_{\mathscr{A} A_{j}^{\prime}\left(A_{2}^{\prime} \ldots A_{1}^{\prime} \ldots A_{r}^{\prime}\right)}+\cdots=0$, and so

$$
(r-l) f_{\mathscr{A} 0^{\prime} 0^{\prime} \ldots 0^{\prime} 1^{\prime} 1^{\prime} \ldots}+l f_{\mathscr{A} 1^{\prime} 0^{\prime} \ldots 0^{\prime} 0^{\prime} 1^{\prime} \ldots}=0 .
$$

Assume that $A_{1}^{\prime}=0^{\prime}$ in (4.18). Then

$$
\begin{aligned}
\left.\varepsilon_{0^{\prime}\left(A_{2}^{\prime}\right.} f_{|\mathscr{A}|} C^{C^{\prime}} A_{3}^{\prime} \ldots A_{r}^{\prime}\right) C^{\prime} & =\frac{l}{r-1} \varepsilon_{0^{\prime} 1^{\prime}} f_{\mathscr{A}} C_{0^{\prime} \ldots 0^{\prime} 1^{\prime} \ldots 1^{\prime} C^{\prime}}=\frac{l}{r-1}\left(-f_{\mathscr{A} 0^{\prime} 0^{\prime} \ldots 0^{\prime} 1^{\prime} \ldots 1^{\prime} 1^{\prime}}+f_{\mathscr{A} 1^{\prime} 0^{\prime} \ldots 0^{\prime} 1^{\prime} \ldots 1^{\prime} 0^{\prime}}\right) \\
& =-\frac{r}{r-1} f_{\mathscr{A} 0^{\prime} 0^{\prime} \ldots 0^{\prime} 1^{\prime} \ldots 1^{\prime} 1^{\prime}}
\end{aligned}
$$

by using

$$
f_{\ldots}^{B^{\prime}} \ldots B^{\prime} \ldots=-f_{\ldots 0^{\prime} \ldots 1^{\prime} \ldots}+f_{\ldots 1^{\prime} \ldots 0^{\prime} \ldots}=-f_{\ldots B^{\prime} \ldots{ }^{B^{\prime}} \ldots} .
$$

in the second identity and (4.19) in the last identity. It is similar for $A_{1}^{\prime}=1^{\prime}$.

Proof of Proposition 4.1. Since $D_{j}^{(k)} f=0$, i.e. $\mathscr{D}_{q, p} f=0$ for $q=j, p=k-j$, we have

$$
0=\left(\mathscr{D}_{q, p} f, \mathscr{D}_{q, p} f\right)=\left(\mathscr{D}_{q, p}^{*} \mathscr{D}_{q, p} f, f\right) .
$$

Then by choose local orthonormal quaternionic frame as before, locally we have $\left(\mathscr{D}_{q, p}^{*} \mathscr{D}_{q, p} f\right)_{B_{1} \ldots B_{q} B_{1}^{\prime} \ldots B_{p}^{\prime}}=$ $\nabla_{\left(B_{1}^{\prime}\right.}^{A} \nabla_{\mid[A}^{A^{\prime}} f_{\left.\left.B_{1} \ldots B_{q}\right] A^{\prime} \mid B_{2}^{\prime} \ldots B_{p}^{\prime}\right)}$. To calculate

$$
\left(\nabla_{\left(B_{1}^{\prime}\right.}^{A} \nabla_{\mid[A}^{A^{\prime}} f_{\left.\left.B_{1} \ldots B_{q}\right] A^{\prime} \mid B_{2}^{\prime} \ldots B_{p}^{\prime}\right)}, f_{B_{1} \ldots B_{q} B_{1}^{\prime} B_{2}^{\prime} \ldots B_{p}^{\prime}}\right),
$$

we only need to calculate the term without symmetrisation by (4.15), i.e.

$$
\nabla_{B_{1}^{\prime}}^{A} \nabla_{[A}^{A^{\prime}} f_{\left.B_{1} \ldots B_{q}\right] A^{\prime} B_{2}^{\prime} \ldots B_{p}^{\prime}}=\varepsilon_{B^{\prime} B_{1}^{\prime}}\left(\nabla^{A\left[B^{\prime}\right.} \nabla_{[A}^{\left.A^{\prime}\right]}+\nabla^{A\left(B^{\prime}\right.} \nabla_{[A}^{\left.A^{\prime}\right)}\right) f_{\left.B_{1} \ldots B_{q}\right] A^{\prime} B_{2}^{\prime} \ldots B_{p}^{\prime}} .
$$

For the first term in the right hand side, note that

$$
\nabla^{A\left[B^{\prime}\right.} \nabla_{C}^{\left.A^{\prime}\right]}=\varepsilon_{B^{\prime} A^{\prime}} \nabla^{A\left[0^{\prime}\right.} \nabla_{C}^{\left.1^{\prime}\right]}=\frac{\varepsilon_{B^{\prime} A^{\prime}}}{2}\left(\nabla^{A 0^{\prime}} \nabla_{C}^{1^{\prime}}-\nabla^{A 1^{\prime}} \nabla_{C}^{0^{\prime}}\right)=-\frac{\varepsilon_{B^{\prime} A^{\prime}}}{2}\left(\nabla^{A 0^{\prime}} \nabla_{C 0^{\prime}}+\nabla^{A 1^{\prime}} \nabla_{C 1^{\prime}}\right),
$$


by $\varepsilon_{0^{\prime} 1^{\prime}}=1$, and $\sum_{B^{\prime}=0^{\prime}, 1^{\prime}} \varepsilon_{B^{\prime} A^{\prime}} \varepsilon_{B^{\prime} B_{1}^{\prime}}=\delta_{A^{\prime} B_{1}^{\prime}}$. If we display the antisymmetrisation of the unprimed indices in the second term in (4.22), we see that

$$
\begin{aligned}
& \nabla_{B_{1}^{\prime}}^{A} \nabla_{[A}^{A^{\prime}} f_{\left.B_{1} \ldots B_{q}\right] A^{\prime} B_{2}^{\prime} \ldots B_{p}^{\prime}}=-\frac{1}{2} \nabla^{A C^{\prime}} \nabla_{C^{\prime}[A} f_{\left.B_{1} \ldots B_{q}\right] B_{1}^{\prime} \ldots B_{p}^{\prime}} \\
& +\frac{\varepsilon_{B^{\prime} B_{1}^{\prime}}}{q+1}\left(\nabla^{A\left(B^{\prime}\right.} \nabla_{A}^{\left.A^{\prime}\right)} f_{B_{1} \ldots B_{q} A^{\prime} B_{2}^{\prime} \ldots B_{p}^{\prime}}+\sum_{s=1}^{q}(-1)^{s} \nabla^{A\left(B^{\prime}\right.} \nabla_{B_{s}}^{\left.A^{\prime}\right)} f_{\ldots A} \ldots A^{\prime} B_{2}^{\prime} \ldots B_{p}^{\prime}\right) \\
& =-\frac{1}{2} \nabla^{A C^{\prime}} \nabla_{C^{\prime}[A} f_{\left.B_{1} \ldots B_{q}\right] B_{1}^{\prime} \ldots B_{p}^{\prime}}+\frac{\varepsilon_{B^{\prime} B_{1}^{\prime}}}{q+1} \nabla^{A\left(B^{\prime}\right.} \nabla_{A}^{\left.A^{\prime}\right)} f_{B_{1} \ldots B_{q} A^{\prime} B_{2}^{\prime} \ldots B_{p}^{\prime}} \\
& +\frac{\varepsilon_{B^{\prime} B_{1}^{\prime}}}{q+1}\left(\sum_{s=1}^{q}(-1)^{s}\left(\nabla^{A\left(B^{\prime}\right.} \nabla_{B_{s}}^{\left.A^{\prime}\right)}-\nabla_{B_{s}}^{\left(A^{\prime}\right.} \nabla^{\left.B^{\prime}\right) A}\right)\right. \\
& \left.\quad+\frac{1}{2} \sum_{s=1}^{q}(-1)^{s} \nabla_{B_{s}}^{B^{\prime}} \nabla^{A^{\prime} A}+\frac{1}{2} \sum_{s=1}^{q}(-1)^{s} \nabla_{B_{s}}^{A^{\prime}} \nabla^{B^{\prime} A}\right) f_{B_{1} \ldots A B_{q} A^{\prime} B_{2}^{\prime} \ldots B_{p}^{\prime}} \\
& :=\left(S_{1} f+S_{2} f+S_{3} f+S_{4} f+S_{5} f\right)_{B_{1} \ldots B_{q} B_{1}^{\prime} \ldots B_{p}^{\prime} .}
\end{aligned}
$$

Now we get $0=\left(S_{1} f, f\right)+\ldots+\left(S_{5} f, f\right)$ by (4.21). The reason we use the expansion above is that $S_{2} f$ and $S_{3} f$ are commutators of the form $\nabla_{[A}^{\left(A^{\prime}\right.} \nabla_{B]}^{\left.B^{\prime}\right)}$, which are curvature terms. Obviously by using (4.14) and Proposition 4.3 , we have

$$
\left(S_{1} f, f\right)=\frac{1}{2}\left(\nabla^{*} \widehat{\nabla} f, f\right)=\frac{1}{2}(\widehat{\nabla} f, \nabla f)=\frac{1}{2}(\widehat{\nabla} f, \widehat{\nabla} f)
$$

by antisymmetrisation (4.14) in the second identity.

Recall that $\Phi=0$ for quaternionic Kähler manifold, and so we have

$$
R_{A B C}^{\left(A^{\prime} B^{\prime}\right)}{ }^{D}=0, \quad R_{A B}{ }^{A^{\prime} B^{\prime}}{ }_{C^{\prime}}^{D^{\prime}}=2 \Lambda_{A B} \delta_{C^{\prime}}{ }^{\left(A^{\prime}\right.} \varepsilon^{\left.B^{\prime}\right) D^{\prime}}
$$

by Corollary 3.1 and Proposition 2.2 . Note that

$$
\begin{aligned}
& \varepsilon_{B^{\prime} B_{1}^{\prime}} \nabla_{[C}^{\left(B^{\prime}\right.} \nabla_{A]}^{\left.B_{0}^{\prime}\right)} f_{B_{1} \ldots B_{q} B_{0}^{\prime} B_{2}^{\prime} \ldots B_{p}^{\prime}}=-\frac{1}{2} \varepsilon_{B^{\prime} B_{1}^{\prime}}\left(R_{[C A]}^{B^{\prime} B_{0}^{\prime}}{ }_{B_{j}^{\prime}}^{D^{\prime}} f_{B_{1} \ldots B_{q} B_{0}^{\prime} \ldots D^{\prime} \ldots B_{p}^{\prime}}\right. \\
& \left.+R^{\left(B^{\prime} B_{0}^{\prime}\right)}{ }_{C A B_{j}}{ }^{D} f_{B_{1} \ldots D \ldots B_{q} B_{0}^{\prime} \ldots B_{p}^{\prime}}\right) \\
& =-\varepsilon_{B^{\prime} B_{1}^{\prime}} \Lambda_{C A} \delta_{B_{j}^{\prime}}{ }^{\left(B^{\prime}\right.} \varepsilon^{\left.B_{0}^{\prime}\right) D^{\prime}} f_{B_{1} \ldots B_{q} \ldots D^{\prime} \ldots B_{p}^{\prime}} \\
& =\frac{p+2}{2} \epsilon_{C A} \Lambda f_{B_{1} \ldots B_{q} B_{1}^{\prime} B_{2}^{\prime} \ldots B_{p}^{\prime}}
\end{aligned}
$$

by using (3.5) for the commutator, Proposition 2.3 for $\Lambda_{A B}$ and (4.25), and for $j=2, \ldots, p$,

$$
\begin{aligned}
2 \varepsilon_{B^{\prime} B_{1}^{\prime}} \delta_{B_{j}^{\prime}}{ }^{\left(B^{\prime}\right.} \varepsilon^{\left.B_{0}^{\prime}\right) D^{\prime}} f_{B_{1} \ldots B_{q} B_{0}^{\prime} \ldots D^{\prime} \ldots B_{p}^{\prime}} & =\left(\varepsilon_{B_{j}^{\prime} B_{1}^{\prime}} \varepsilon^{B_{0}^{\prime} D^{\prime}}+\delta_{B_{j}^{\prime}}{ }^{B_{0}^{\prime}} \varepsilon_{B^{\prime} B_{1}^{\prime}} \varepsilon^{B^{\prime} D^{\prime}}\right) f_{B_{1} \ldots B_{q} B_{0}^{\prime} \ldots D^{\prime} \ldots B_{p}^{\prime}} \\
& =-f_{B_{1} \ldots B_{q} B_{j}^{\prime} \ldots B_{1}^{\prime} \ldots B_{p}^{\prime}}
\end{aligned}
$$

since $\varepsilon^{B_{0}^{\prime} D^{\prime}}$ is antisymmetric and $f \ldots$ is symmetric in primed indices, while for $j=0$,

$$
\begin{aligned}
2 \varepsilon_{B^{\prime} B_{1}^{\prime}} \delta_{B_{0}^{\prime}}{ }^{\left(B^{\prime}\right.} \varepsilon^{\left.B_{0}^{\prime}\right) D^{\prime}} f_{B_{1} \ldots B_{q} D^{\prime} B_{2}^{\prime} \ldots B_{p}^{\prime}} & =\left(\varepsilon_{B_{0}^{\prime} B_{1}^{\prime}} \varepsilon^{B_{0}^{\prime} D^{\prime}}+\delta_{B_{0}^{\prime}}^{B_{0}^{\prime}} \varepsilon_{B^{\prime} B_{1}^{\prime}} \varepsilon^{B^{\prime} D^{\prime}}\right) f_{B_{1} \ldots B_{q} D^{\prime} B_{2}^{\prime} \ldots B_{p}^{\prime}} \\
& =-3 f_{B_{1} \ldots B_{q} B_{1}^{\prime} B_{2}^{\prime} \ldots B_{p}^{\prime}} .
\end{aligned}
$$

Then, lowering the superscript $A$ in $S_{2} f$ and applying (4.26) to $S_{2} f$, we get

$$
\left(S_{2} f\right)_{B_{1} \ldots B_{q} B_{1}^{\prime} \ldots B_{p}^{\prime}}=-\frac{p+2}{q+1} n \Lambda f_{B_{1} \ldots B_{q} B_{1}^{\prime} \ldots B_{p}^{\prime}}
$$


and similarly, applying (4.26) to $S_{3} f$, we get

$$
\begin{aligned}
\left(S_{3} f\right)_{B_{1} \ldots B_{q} B_{1}^{\prime} \ldots B_{p}^{\prime}} & =2 \sum_{s=1}^{q} \frac{(-1)^{s} \epsilon^{C A}}{q+1} \varepsilon_{B^{\prime} B_{1}^{\prime}} \nabla_{[C}^{\left(B^{\prime}\right.} \nabla_{\left.B_{s}\right]}^{\left.B_{0}^{\prime}\right)} f_{B_{1} \ldots A} \ldots B_{q} B_{0}^{\prime} B_{2}^{\prime} \ldots B_{p}^{\prime} \\
& =\sum_{s=1}^{q} \frac{p+2}{q+1}(-1)^{s} \epsilon^{C A} \epsilon_{C B_{s}} \Lambda f_{B_{1} \ldots A \ldots B_{q} B_{1}^{\prime} \ldots B_{p}^{\prime}}=\frac{1-(-1)^{q}}{2} \frac{p+2}{q+1} \Lambda f_{B_{1} \ldots B_{q} B_{1}^{\prime} \ldots B_{p}^{\prime}} .
\end{aligned}
$$

Hence

$$
\left(S_{2} f, f\right)+\left(S_{3} f, f\right)=-\frac{2 n-1+(-1)^{q}}{2(q+1)}(p+2)(\Lambda f, f) .
$$

Similarly we have

$$
\begin{aligned}
\left(S_{4} f\right)_{B_{1} \ldots B_{q} B_{1}^{\prime} \ldots B_{p}^{\prime}} & =\sum_{s=1}^{q} \frac{-1}{2(q+1)} \nabla_{B_{s} B_{1}^{\prime}} \nabla^{A^{\prime} A} f_{A B_{1} \ldots \widehat{B}_{s} \ldots B_{q} A^{\prime} B_{2}^{\prime} \ldots B_{p}^{\prime}} \\
& =\frac{1}{2(q+1)} \sum_{s=1}^{q}\left(\nabla \nabla^{*} f\right)_{B_{s} B_{1} \ldots \widehat{B}_{s} \ldots B_{q} B_{1}^{\prime} B_{2}^{\prime} \ldots B_{p}^{\prime}}
\end{aligned}
$$

by $f$ antisymmetric in unprimed indices and the expression of $\nabla^{*}$ in Proposition 4.3 , and

$$
\begin{aligned}
& \left(S_{5} f\right)_{B_{1} \ldots B_{q} B_{1}^{\prime} \ldots B_{p}^{\prime}}=-\sum_{s=1}^{q} \frac{1}{2(q+1)} \nabla_{B_{s}}^{A^{\prime}} \nabla_{B_{1}^{\prime}}^{A} f_{A B_{1} \ldots \widehat{B}_{s} \ldots B_{q} A^{\prime} B_{2}^{\prime} \ldots B_{p}^{\prime}} \\
& =\sum_{s=1}^{q} \frac{1}{2(q+1)} \frac{p}{p+1} \nabla_{B_{s}}^{A^{\prime}} \varepsilon_{B_{1}^{\prime}\left(A^{\prime}\right.} \nabla^{A C^{\prime}} f_{\left.\left|A B_{1} \ldots \widehat{B}_{s} \ldots B_{q}\right| B_{2}^{\prime} \ldots B_{p}^{\prime}\right) C^{\prime}} \\
& =\sum_{s=1}^{q} \frac{1}{2(q+1)} \frac{1}{p+1}\left[-\nabla_{B_{s} B_{1}^{\prime}} \nabla^{A C^{\prime}} f_{A B_{1} \ldots \widehat{B}_{s} \ldots B_{q} B_{2}^{\prime} \ldots B_{p}^{\prime} C^{\prime}}+\varepsilon_{B_{1}^{\prime} B_{j}^{\prime}} \nabla_{B_{s}}^{A^{\prime}} \nabla^{A C^{\prime}} f_{A B_{1} \ldots \widehat{B}_{s} \ldots B_{q} B_{2}^{\prime} \ldots A^{\prime} \ldots B_{p}^{\prime} C^{\prime}}\right] \\
& =\frac{1}{2(q+1)(p+1)} \sum_{s=1}^{q}\left(\nabla \nabla^{*} f\right)_{B_{s} B_{1} \ldots \widehat{B}_{s} \ldots B_{q} B_{1}^{\prime} B_{2}^{\prime} \ldots B_{p}^{\prime}}+\widetilde{S}_{5} f
\end{aligned}
$$

where in the second identity we apply Lemma 4.1 to $\nabla_{B_{1}^{\prime}}^{A} f_{B_{1} \ldots A} \ldots B_{q} A^{\prime} B_{2}^{\prime} \ldots B_{p}^{\prime}$ with $r=p+1$, since the condition of this lemma is satisfied by (4.16). Note that $\left(\widetilde{S}_{5} f, f\right)=0$ by $f$ symmetric but $\varepsilon_{B_{1}^{\prime} B_{j}^{\prime}}$ antisymmetric in $B_{1}^{\prime}, B_{j}^{\prime}$. Therefore

$$
\left(S_{4} f, f\right)+\left(S_{5} f, f\right)=\frac{(p+2)}{2(q+1)(p+1)} \sum_{s=1}^{q}(-1)^{s-1}\left(\nabla^{*} f, \nabla^{*} f\right)=\frac{\left(1-(-1)^{q}\right)(p+2)}{4(q+1)(p+1)}\left(\nabla^{*} f, \nabla^{*} f\right) .
$$

Substituting (4.24), (4.28) and (4.29) into $0=\left(S_{1} f, f\right)+\ldots+\left(S_{5} f, f\right)$, we get the identity (4.2).

\section{Appendix A. Proof of some Propositions}

At first, the traces of curvatures vanish when we antisymmetrise primed (or unprimed) indices and symmetrise unprimed (or primed) indices.

Proposition A.1. The curvatures of a unimodular quaternionic manifold satisfy

$$
\begin{aligned}
R_{\left[A^{\prime} B^{\prime}\right] C(A B)}{ }^{C} & =0, & R_{\left(A^{\prime} B^{\prime}\right) C[A B]}{ }^{C}=0, \\
R_{[A B] C^{\prime}\left(A^{\prime} B^{\prime}\right)} C^{\prime} & =0, & R_{(A B) C^{\prime}\left[A^{\prime} B^{\prime}\right]} C^{\prime}=0 .
\end{aligned}
$$


Proof. The first Bianchi identity

$$
R_{[a b c]}^{d}=0,
$$

and the antisymmetry of $R_{a b c}{ }^{d}$ in $a$ and $b$ implies the cyclicity:

$$
R_{A A^{\prime} B B^{\prime} C C^{\prime}} D D^{\prime}+R_{B B^{\prime} C C^{\prime} A A^{\prime}}^{D D^{\prime}}+R_{C C^{\prime} A A^{\prime} B B^{\prime}}^{D D^{\prime}}=0
$$

for all $A, \ldots, A^{\prime}, \ldots$ Take trace over $C^{\prime}-D^{\prime}$ in (A.3) to get

$$
2 R_{A^{\prime} B^{\prime} A B C}{ }^{D}+R_{B^{\prime} A^{\prime} B C A}{ }^{D}+R_{B^{\prime} A^{\prime} C A B}{ }^{D}+R_{B C B^{\prime} C^{\prime} A^{\prime}} C^{\prime} \delta_{A}^{D}+R_{C A C^{\prime} A^{\prime} B^{\prime}} C^{\prime} \delta_{B}{ }^{D}=0 .
$$

by the decomposition (2.21) of curvatures. Here and in the sequel we use vanishing of traces in (2.22) repeatedly.

Taking trace over $C$ - $D$ in (A.4), we get

$$
R_{B^{\prime} A^{\prime} B C A}{ }^{C}+R_{B^{\prime} A^{\prime} C A B}{ }^{C}+R_{B A B^{\prime} C^{\prime} A^{\prime}} C^{\prime}+R_{B A C^{\prime} A^{\prime} B^{\prime}} C^{\prime}=0,
$$

by using (2.22) again. Antisymmetrising $\left[A^{\prime} B^{\prime}\right]$ and symmetrising $(A B)$ in (A.5), we get

$$
R_{\left[B^{\prime} A^{\prime}\right] C(A B)}{ }^{C}+R_{(A B) C^{\prime}\left[A^{\prime} B^{\prime}\right]}{ }^{C^{\prime}}=0,
$$

by using

$$
R_{\left[B^{\prime} A^{\prime}\right] B C A}{ }^{D}=R_{\left[B^{\prime} A^{\prime}\right] C B A}{ }^{D} \quad \text { and } \quad R_{(B A) B^{\prime} C^{\prime} A^{\prime}}{ }^{D^{\prime}}=-R_{(B A) C^{\prime} B^{\prime} A^{\prime}}{ }^{D^{\prime}}
$$

which follows from antisymmetry

$$
R_{A^{\prime} B^{\prime} A B C}{ }^{D}=-R_{B^{\prime} A^{\prime} B A C}{ }^{D}, \quad R_{A B A^{\prime} B^{\prime} C^{\prime}}^{D^{\prime}}=-R_{B A B^{\prime} A^{\prime} C^{\prime}}^{D^{\prime}},
$$

i.e., $\quad R_{a b C}{ }^{D}=-R_{b a C}{ }^{D}$ and $\quad R_{a b C^{\prime}}{ }^{D^{\prime}}=-R_{b a C^{\prime}}{ }^{D^{\prime}}$. Here (A.7) means that $R_{\left[B^{\prime} A^{\prime}\right] B C A}{ }^{D}$ is symmetric in $B, C$, while $R_{(B A) B^{\prime} C^{\prime} A^{\prime}} D^{\prime}$ is antisymmetric in $B^{\prime}, C^{\prime}$. These identities will be used frequently to change the order of indices.

Taking trace over $B$ - $D$ in (A.4), we get

$$
2 R_{A^{\prime} B^{\prime} A B C}{ }^{B}+R_{B^{\prime} A^{\prime} B C A}{ }^{B}+R_{A C B^{\prime} C^{\prime} A^{\prime}} C^{\prime}+2 n R_{C A C^{\prime} A^{\prime} B^{\prime}}{ }^{C^{\prime}}=0 .
$$

Antisymmetrising $\left[A^{\prime} B^{\prime}\right]$ and symmetrising $(A C)$ in $(\underline{A .9}$, we see that

$$
R_{\left[A^{\prime} B^{\prime}\right] B(A C)}{ }^{B}+(2 n+1) R_{(A C) C^{\prime}\left[A^{\prime} B^{\prime}\right]}{ }^{C^{\prime}}=0,
$$

by (A.7). Now (A.6) and (A.10) imply that the first and the last identities in (A.1).

Symmetrising $\left(A^{\prime} B^{\prime}\right)$ and antisymmetrising $[A B]$ in (A.5), we get

$$
R_{\left(B^{\prime} A^{\prime}\right) C[A B]}{ }^{C}-R_{[A B] C^{\prime}\left(B^{\prime} A^{\prime}\right)}{ }^{C^{\prime}}=0 .
$$

On the other hand, we take trace over $C$ - $D$ in (A.3) to get

$$
R_{B^{\prime} C^{\prime} B C A}{ }^{C} \delta_{A^{\prime}}^{D^{\prime}}+R_{C^{\prime} A^{\prime} C A B}{ }^{C} \delta_{B^{\prime}}^{D^{\prime}}+2 n R_{A B A^{\prime} B^{\prime} C^{\prime}}^{D^{\prime}}+R_{B A B^{\prime} C^{\prime} A^{\prime}} D^{\prime}+R_{B A C^{\prime} A^{\prime} B^{\prime}} D^{\prime}=0 .
$$

Taking trace over $B^{\prime}-D^{\prime}$ in (A.12), we get

$$
R_{A^{\prime} C^{\prime} B C A}{ }^{C}+2 R_{C^{\prime} A^{\prime} C A B}{ }^{C}+2 n R_{A B A^{\prime} B^{\prime} C^{\prime}}{ }^{B^{\prime}}+R_{B A B^{\prime} C^{\prime} A^{\prime}}{ }^{B^{\prime}}=0 .
$$

Then symmetrising $\left(A^{\prime} C^{\prime}\right)$ and antisymmetrising $[A B]$ in (A.13), we get

$$
3 R_{\left(A^{\prime} C^{\prime}\right) C[A B]}^{C}+(2 n-1) R_{[A B] B^{\prime}\left(A^{\prime} C^{\prime}\right)} B^{B^{\prime}}=0,
$$

by using (A.7) again. This together with (A.11) implies that the second and the third identity in (A.1). The proposition is proved. 
Proof of Proposition 2.2. (1) The first identity in (2.24) follows directly from the definition of $\Psi$ in (2.25).

(2) Now antisymmetrising $\left[A^{\prime} B^{\prime}\right]$ and $[A C]$ in $(\underline{A .9}$, we see that

$$
3 R_{\left[A^{\prime} B^{\prime}\right] B[A C]}^{B}-(2 n+1) R_{[A C] C^{\prime}\left[A^{\prime} B^{\prime}\right]}^{C^{\prime}}=0
$$

by using A.7) again. Namely

$$
R_{\left[A^{\prime} B^{\prime}\right] C[A B]}^{C}=(2 n+1) \Lambda_{A B} \varepsilon_{A^{\prime} B^{\prime}}
$$

by the definition of $\Lambda$ in (2.25). On the other hand symmetrising $\left(A^{\prime} B^{\prime}\right)$ and $(A C)$ in (A.9), we get

$$
-R_{\left(A^{\prime} B^{\prime}\right) B(A C)}^{B}+(2 n-1) R_{(A C) C^{\prime}\left(A^{\prime} B^{\prime}\right)}{ }^{C^{\prime}}=0 .
$$

Then by the definition of $\Phi$ in (2.25), we get

$$
R_{\left(A^{\prime} B^{\prime}\right) C(A B)}{ }^{C}=(2 n-1) \Phi_{A B A^{\prime} B^{\prime}}
$$

(3) Antisymmetrising $\left[A^{\prime} B^{\prime}\right]$ and symmetrising $(A B)$ in A.4 , we get

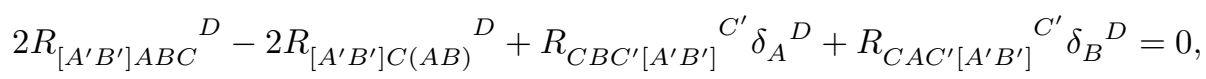

by using the antisymmetry (A.8). It follows from the last identity in (A.1) and the definition of $\Lambda$ in (2.25) that

$$
R_{C B C^{\prime}\left[A^{\prime} B^{\prime}\right]}^{C^{\prime}} \delta_{A}^{D}=R_{[C B] C^{\prime}\left[A^{\prime} B^{\prime}\right]}{ }^{C^{\prime}} \delta_{A}^{D}=3 \Lambda_{C B} \delta_{A}{ }^{D} \varepsilon_{A^{\prime} B^{\prime}}
$$

Thus the sum of the last two terms in (A.16) is equal to $6 \Lambda_{C(B} \delta_{A)}{ }^{D}$. Then by the trivial identity $2 \Lambda_{C(B} \delta_{A)}{ }^{D}=\delta_{(C}{ }^{D} \Lambda_{A) B}+\delta_{(C}{ }^{D} \Lambda_{B) A}$ and the definition of $\Psi$ in (2.25), we see that (A.16) is equivalent to

$$
\Psi_{A B C}^{D}=\Psi_{C(A B)}{ }^{D}
$$

Then $\Psi_{A B C}{ }^{D}=\Psi_{(A B C)}{ }^{D}$ since $\Psi_{A B C}{ }^{D}$ is symmetric in $A, B$ by definition. $\Psi_{A B C}{ }^{A}=0$ by symmetrising $(B C)$ in the definition (2.25) of $\Psi$ and using the first identity in (A.1).

(4) Symmetrise $\left(A^{\prime} B^{\prime}\right)$ and antisymmetrise $[A B]$ in (A.4) to get

$$
2 R_{\left(A^{\prime} B^{\prime}\right) A B C}{ }^{D}+2 R_{\left(A^{\prime} B^{\prime}\right) C[A B]}{ }^{D}-R_{C B C^{\prime}\left(A^{\prime} B^{\prime}\right)}{ }^{C^{\prime}} \delta_{A}^{D}+R_{C A C^{\prime}\left(A^{\prime} B^{\prime}\right)}{ }^{C^{\prime}} \delta_{B}^{D}=0 .
$$

Apply (A.1) and the definition of $\Phi$ in (2.25) to the last two terms above to get

$$
R_{\left(A^{\prime} B^{\prime}\right) A B C}{ }^{D}+R_{\left(A^{\prime} B^{\prime}\right) C[A B]}^{D}-\delta_{[A}{ }^{D} \Phi_{B] C A^{\prime} B^{\prime}}=0
$$

Now antisymmetrising $[A B C]$ above, we find that

$$
R_{\left(A^{\prime} B^{\prime}\right)[A B C]}^{D}=0
$$

which is equivalent to

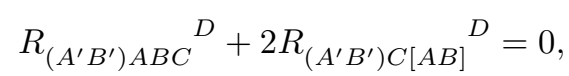

by $R_{\left(A^{\prime} B^{\prime}\right) A B C}{ }^{D}$ antisymmetric in $A, B$. Substitute this into (A.17) to get the second identity in (2.24).

(5) To show the last identity in (2.24), antisymmetrise $[A B]$ in (A.12) to get

$$
-R_{C^{\prime} B^{\prime} C[B A]}{ }^{C} \delta_{A^{\prime}} D^{\prime}+R_{C^{\prime} A^{\prime} C[A B]}{ }^{C} \delta_{B^{\prime}}^{D^{\prime}}+2 n R_{[A B] A^{\prime} B^{\prime} C^{\prime}}^{D^{\prime}}-2 R_{[A B] C^{\prime}\left(A^{\prime} B^{\prime}\right)}^{D^{\prime}}=0
$$


by using (A.7) to the first term. Then we have

$$
(2 n+1) \Lambda_{A B}\left(\varepsilon_{C^{\prime} B^{\prime}} \delta_{A^{\prime}}^{D^{\prime}}+\varepsilon_{C^{\prime} A^{\prime}} \delta_{B^{\prime}} D^{\prime}\right)+2 n R_{[A B] A^{\prime} B^{\prime} C^{\prime}} D^{\prime}-2 R_{[A B] C^{\prime}\left(A^{\prime} B^{\prime}\right)} D^{D^{\prime}}=0,
$$

by (A.1) and (A.14). Symmetrising $\left(A^{\prime} B^{\prime} C^{\prime}\right)$ in the above identity, we get

$$
(2 n-2) R_{[A B]\left(A^{\prime} B^{\prime} C^{\prime}\right)} D^{\prime}=0 .
$$

If $n \neq 1$, it is equivalent to

$$
R_{[A B] A^{\prime} B^{\prime} C^{\prime}}^{D^{\prime}}+2 R_{[A B] C^{\prime}\left(A^{\prime} B^{\prime}\right)}{ }^{D^{\prime}}=0,
$$

by $R_{[A B] A^{\prime} B^{\prime} C^{\prime}} D^{\prime}$ symmetric in $A^{\prime}$ and $B^{\prime}$. Substitute this into (A.19) to get the last identity in (2.24). If $n=1$, (2.27) follows from the definition of $\Psi_{A^{\prime} B^{\prime} C^{\prime}}^{\prime} D^{\prime}$. We have $\Psi_{A^{\prime} B^{\prime} C^{\prime}}^{\prime} D^{\prime}=\Psi_{\left(A^{\prime} B^{\prime} C^{\prime}\right)}^{\prime} D^{\prime}$ as in part (3) by exchanging the primed and unprimed indices.

(6) At last, we symmetrise $(A B)$ in (A.12) to get

$$
-R_{C^{\prime} B^{\prime} C(A B)}{ }^{C} \delta_{A^{\prime}}{ }^{D^{\prime}}+R_{C^{\prime} A^{\prime} C(A B)}{ }^{C} \delta_{B^{\prime}}^{D^{\prime}}+2 n R_{(A B) A^{\prime} B^{\prime} C^{\prime}}^{D^{\prime}}-R_{(A B) C^{\prime} B^{\prime} A^{\prime}}{ }^{D^{\prime}}+R_{(A B) C^{\prime} A^{\prime} B^{\prime}}{ }^{D^{\prime}}=0,
$$

by using (A.7) again. By (A.1), A.15) and the second identity in (2.24), we find that

$$
(2 n-1)\left(-\Phi_{A B C^{\prime} B^{\prime}} \delta_{A^{\prime}}^{D^{\prime}}+\Phi_{A B C^{\prime} A^{\prime}} \delta_{B^{\prime}}^{D^{\prime}}\right)+2 n R_{(A B) A^{\prime} B^{\prime} C^{\prime}}^{D^{\prime}}+2 R_{(A B) C^{\prime}\left[A^{\prime} B^{\prime}\right]}{ }^{D^{\prime}}=0 .
$$

Antisymmetrise $\left[A^{\prime} B^{\prime} C^{\prime}\right]$ above to get

$$
(2 n+2) R_{(A B)\left[A^{\prime} B^{\prime} C^{\prime}\right]}^{D^{\prime}}=0 .
$$

Namely

$$
R_{(A B) A^{\prime} B^{\prime} C^{\prime}}{ }^{D^{\prime}}+2 R_{(A B) C^{\prime}\left[A^{\prime} B^{\prime}\right]}{ }^{D^{\prime}}=0 .
$$

Substitute this into (A.22) to get

$$
R_{(A B) A^{\prime} B^{\prime} C^{\prime}}^{D^{\prime}}=\Phi_{A B C^{\prime} B^{\prime}} \delta_{A^{\prime}}^{D^{\prime}}-\Phi_{A B C^{\prime} A^{\prime}} \delta_{B^{\prime}}^{D^{\prime}} .
$$

The third identity in (2.24) is obtained. The proposition is proved.

Proof of Proposition 2.3. (1) By the second Bianchi identity

$$
\nabla_{[a} R_{b c] d}^{e}=0 .
$$

for $a=A A^{\prime}, b=B A^{\prime}, c=C A^{\prime}$ and $d=D D^{\prime}, e=D A^{\prime}$, and taking summation over repeated indices, we find that

$$
\nabla_{A^{\prime}[A} R_{\left.\left|A^{\prime} A^{\prime}\right| B C\right] D}{ }^{D} \delta_{D^{\prime}}{ }^{A^{\prime}}+\nabla_{A^{\prime}[A} R_{B C] A^{\prime} A^{\prime} D^{\prime}}{ }^{A^{\prime}} \delta_{D}{ }^{D}=0 .
$$

The first term vanishes as a trace by (2.22), while the second term is $2 \nabla_{A^{\prime}\left[{ }_{A}\right.} \Lambda_{B C]} \varepsilon_{A^{\prime} D^{\prime}} \delta_{D}{ }^{D}$ by the last identity of (2.24) and (2.27) for the 4-dimensional case.

(2) Note that $\nabla_{B B^{\prime}} \epsilon_{C D}=0$ implies $R_{\left(A^{\prime} B^{\prime}\right) A B C}{ }^{E} \epsilon_{E D}+R_{\left(A^{\prime} B^{\prime}\right) A B D}{ }^{E} \epsilon_{C E}=0$, or equivalently

$$
-\epsilon_{D[A} \Phi_{B] C A^{\prime} B^{\prime}}+\epsilon_{C[A} \Phi_{B] D A^{\prime} B^{\prime}}=0 .
$$

by Proposition 2.2. If we antisymmetrise $[D A B]$, we get $\epsilon_{[D A} \Phi_{B] C A^{\prime} B^{\prime}}=0$, which can hold only if $\Phi_{A B A^{\prime} B^{\prime}}=0$. 
The Ricci curvature of the connection on tangent bundle is given by

$$
\begin{aligned}
& R_{a c}=R_{a b c d} g^{b d}=R_{A A^{\prime} B B^{\prime} C C^{\prime} D D^{\prime}} \epsilon^{B D} \varepsilon^{B^{\prime} D^{\prime}}=R_{A A^{\prime} B B^{\prime} C C^{\prime}}^{B B^{\prime}} \\
& =R_{A^{\prime} B^{\prime} A B C}{ }^{B} \delta_{C^{\prime}}^{B^{\prime}}+R_{A B A^{\prime} B^{\prime} C^{\prime}}{ }^{B^{\prime}} \delta_{C}^{B}=2(n+2) \Lambda_{A C} \varepsilon_{A^{\prime} C^{\prime}},
\end{aligned}
$$

by the curvature decomposition in Proposition 2.2, if we choose the local orthonormal quaternionic frame $Z_{A A^{\prime}}$ so that the metric is $g_{a b}$ in (2.20). Then

$$
s_{g}=R_{a c} g^{a c}=2(n+2) \Lambda_{A C} \varepsilon_{A^{\prime} C^{\prime}} \epsilon^{A C} \varepsilon^{A^{\prime} C^{\prime}}=8 n(n+2) \Lambda .
$$

It remains to show $\Lambda_{A B}=\Lambda \epsilon_{A B}$. Recall that for any given section $\phi_{A B}$ in $\Gamma\left(\Lambda^{2} E^{*}\right)$ satisfying $\nabla_{a} \phi_{A B}=0$, we must have $\phi_{A B}=\alpha \Lambda_{A B}$ for some constant $\alpha$ when the tensor $\Lambda_{A B} \neq 0$ (this is lemma 7.7 of [5]). This is because $\nabla_{a} \phi_{C D}=0$ implies $R_{\left[A^{\prime} B^{\prime}\right] A B C}{ }^{E} \phi_{E D}+R_{\left[A^{\prime} B^{\prime}\right] A B D}{ }^{E} \phi_{C E}=0$, i.e.

$$
\Psi_{A B C}{ }^{E} \phi_{E D}+\Psi_{A B D}{ }^{E} \phi_{C E}-2 \Lambda_{C(A} \phi_{B) D}+2 \Lambda_{D(A} \phi_{B) C}=0
$$

by the curvatures decomposition in Proposition 2.2. Symmetrising $(A B C)$ followed by antisymmetrising $[C D]$ kills the $\Lambda$ terms, but simply multiplies the $\Psi$ terms by $\frac{2}{3}$. Thus $-2 \Lambda_{C(A} \phi_{B) D}+2 \Lambda_{D(A} \phi_{B) C}=0$, which is essentially just the wedge product of two exterior forms. It is easy to see that it vanishes if and only if $\phi_{A B}$ is a multiple of $\Lambda_{A B}$.

Proof of (3.21). Locally we choose a coordinate chart $U_{\alpha}$ with trivialization $\left.E^{*}\right|_{U_{\alpha}}=U_{\alpha} \times \mathbb{C}^{2 n}$, $\left.H^{*}\right|_{U_{\alpha}}=U \times \mathbb{C}^{2}$, and a two-component local quaternionic frame $\left\{Z_{A A^{\prime}}\right\}$ such that $\varepsilon$ and $\epsilon$ are standard. In particular, $\varepsilon_{0^{\prime} 1^{\prime}}=\varepsilon^{1^{\prime} 0^{\prime}}=1$. By using (3.20) repeatedly, we get

$$
\begin{aligned}
\widetilde{\nabla}_{A A^{\prime}} \widetilde{\nabla}_{B B^{\prime}} f_{C^{\prime}}= & \widetilde{\nabla}_{A A^{\prime}}\left(\nabla_{B B^{\prime}} f_{C^{\prime}}-\Upsilon_{B C^{\prime}} f_{B^{\prime}}\right) \\
= & \nabla_{A A^{\prime}} \nabla_{B B^{\prime}} f_{C^{\prime}}-\nabla_{A A^{\prime}} \Upsilon_{B C^{\prime}} \cdot f_{B^{\prime}}-\Upsilon_{B C^{\prime}} \cdot \nabla_{A A^{\prime}} f_{B^{\prime}} \\
& -\Upsilon_{B A^{\prime}} \nabla_{A B^{\prime}} f_{C^{\prime}}-\Upsilon_{A B^{\prime}} \nabla_{B A^{\prime}} f_{C^{\prime}}-\Upsilon_{A C^{\prime}} \nabla_{B B^{\prime}} f_{A^{\prime}} \\
& +\Upsilon_{B A^{\prime}} \Upsilon_{A C^{\prime}} f_{B^{\prime}}+\Upsilon_{A C^{\prime}} \Upsilon_{B A^{\prime}} f_{B^{\prime}}+\Upsilon_{A B^{\prime}} \Upsilon_{B C^{\prime}} f_{A^{\prime}} .
\end{aligned}
$$

Note that when $A$ and $B, A^{\prime}$ and $B^{\prime}$ are exchanged, the sum of the 3rd and 6th terms, the sum of 4 th and 5 th terms, and the sum of 7 th and 9 th terms in the right hand side above are all invariant. The above identity minus the one with $A$ and $B, A^{\prime}$ and $B^{\prime}$ exchanged becomes

$$
\begin{aligned}
-\widetilde{R}_{A B A^{\prime} B^{\prime} C^{\prime}} D^{\prime}=-R_{A B A^{\prime} B^{\prime} C^{\prime}} D^{\prime} & -\nabla_{A A^{\prime}} \Upsilon_{B C^{\prime}} \delta_{B^{\prime}}{ }^{D^{\prime}}+\nabla_{B B^{\prime}} \Upsilon_{A C^{\prime}} \delta_{A^{\prime}}^{D^{\prime}} \\
& +\Upsilon_{A C^{\prime}} \Upsilon_{B A^{\prime}} \delta_{B^{\prime}}{ }^{D^{\prime}}-\Upsilon_{B C^{\prime}} \Upsilon_{A B^{\prime}} \delta_{A^{\prime}}{ }^{D^{\prime}} .
\end{aligned}
$$

Note that $\Lambda_{A B}=\frac{1}{3} R_{[A B] C^{\prime}\left[0^{\prime} 1^{\prime}\right]} C^{\prime}, \Omega \widetilde{\Lambda}_{A B}=\frac{1}{3} \widetilde{R}_{[A B] C^{\prime}\left[0^{\prime} 1^{\prime}\right]}^{C^{\prime}}$ by definition of $\Lambda$ in (2.25) and

$$
f_{\ldots B^{\prime} \ldots} B^{B^{\prime}} \ldots=f_{\ldots 0^{\prime} \ldots 1^{\prime} \ldots}-f_{\ldots 1^{\prime} \ldots 0^{\prime} \ldots}=-f_{\ldots}^{B^{\prime} \ldots B^{\prime} \ldots} .
$$

Antisymmetrise $[A B]$ and $\left[B^{\prime} C^{\prime}\right]=\left[0^{\prime} 1^{\prime}\right]$ and take trace over $A^{\prime}-D^{\prime}$ in A.25 to get the transformation formula for $\Lambda$. We have used, for example, $\nabla_{0^{\prime}[A} \Upsilon_{B] 1^{\prime}}-\nabla_{1^{\prime}[A} \Upsilon_{B] 0^{\prime}}=\nabla_{A^{\prime}[A} \Upsilon_{B]^{\prime}}^{A^{\prime}}$.

By definition of $\Phi$ in (2.25), symmetrise $\left(B^{\prime} C^{\prime}\right)$ and $(A B)$ and take trace over $A^{\prime}-D^{\prime}$ in (A.25) to get the transformation formula for $\Phi$ in (3.21).

Similarly as (A.25) by exchanging primed and unprimed indices, we get

$$
-\widetilde{R}_{A^{\prime} B^{\prime} A B C}{ }^{D}=-R_{A^{\prime} B^{\prime} A B C}{ }^{D}-\nabla_{A A^{\prime}} \Upsilon_{C B^{\prime}} \delta_{B}^{D}+\nabla_{B B^{\prime}} \Upsilon_{C A^{\prime}} \delta_{A}{ }^{D}+\Upsilon_{C A^{\prime}} \Upsilon_{A B^{\prime}} \delta_{B}{ }^{D}-\Upsilon_{C B^{\prime}} \Upsilon_{B A^{\prime}} \delta_{A}{ }^{D},
$$

from which we get the invariance of $\Psi$ in (3.21) by antisymmetrising $\left[A^{\prime} B^{\prime}\right]=\left[0^{\prime} 1^{\prime}\right]$ and using the transformation formula for $\Lambda$ and the definition of $\Psi$ in (2.25). 
Proof of Proposition 3.1. The new connection $\widetilde{\nabla}$ in (3.20) induces the covariant derivatives for $E$ and $H$ by duality:

$$
\widetilde{\nabla}_{A A^{\prime}} f^{B^{\prime}}=\nabla_{A A^{\prime}} f^{B^{\prime}}+\Theta_{A A^{\prime} D^{\prime}}{ }^{B^{\prime}} f^{D^{\prime}}=\nabla_{A A^{\prime}} f^{B^{\prime}}+\delta_{A^{\prime}}{ }^{B^{\prime}} \Upsilon_{A D^{\prime}} f^{D^{\prime}}
$$

If we raise unprimed indices by $\widetilde{\varepsilon}^{A^{\prime} B^{\prime}}=\Omega^{-1} \varepsilon^{A^{\prime} B^{\prime}}$ (note that $\widetilde{\nabla} \widetilde{\varepsilon}^{A^{\prime} B^{\prime}}=0$ ), we get

$$
\begin{aligned}
\widetilde{\nabla}_{A}^{A^{\prime}} f^{B^{\prime}}: & =\widetilde{\nabla}_{A C^{\prime}} f^{B^{\prime}} \widetilde{\varepsilon}^{C^{\prime} A^{\prime}}=\Omega^{-1}\left(\nabla_{A}^{A^{\prime}} f^{B^{\prime}}-\varepsilon^{A^{\prime} B^{\prime}} \Upsilon_{A D^{\prime}} f^{D^{\prime}}\right), \\
\widetilde{\nabla}_{A}^{A^{\prime}} f_{B}: & :=\widetilde{\nabla}_{A C^{\prime}} f_{B} \widetilde{\varepsilon}^{C^{\prime} A^{\prime}}=\Omega^{-1}\left(\nabla_{A}^{A^{\prime}} f_{B}-\Upsilon_{B}^{A^{\prime}} f_{A}\right), \\
\widetilde{\nabla}_{A}^{A^{\prime}} f_{B^{\prime}}: & =\widetilde{\nabla}_{A C^{\prime}} f_{B^{\prime}} \widetilde{\varepsilon}^{C^{\prime} A^{\prime}}=\Omega^{-1}\left(\nabla_{A}^{A^{\prime}} f_{B^{\prime}}-\Upsilon_{A B^{\prime}} f^{A^{\prime}}\right) .
\end{aligned}
$$

Applying these formulas of covariant derivatives repeatedly, we get

$$
\begin{aligned}
\left(\widetilde{\mathscr{D}}_{q}^{p}\left(\Omega^{-q} f\right)\right)_{A_{1} \ldots A_{q+1}}^{A_{1}^{\prime} \ldots A_{p+1}^{\prime}}= & -q \Omega^{-q-1} \widetilde{\nabla}_{\left[A_{1}\right.}^{\left(A_{1}^{\prime}\right.} \Omega \cdot f_{\left.A_{2} \ldots A_{q+1}\right]}^{\left.A_{2}^{\prime} \ldots A_{p+1}^{\prime}\right)}+\Omega^{-q} \widetilde{\nabla}_{\left[A_{1}\right.}^{\left(A_{1}^{\prime}\right.} f_{\left.A_{2} \ldots A_{q+1}\right]}^{\left.A_{2}^{\prime} \ldots A_{p+1}^{\prime}\right)} \\
= & \Omega^{-q-1}\left\{-q \Upsilon_{\left[A_{1}\right.}^{\left(A_{1}^{\prime}\right.} \cdot f_{\left.A_{2} \ldots A_{q+1}\right]}^{\left.A_{2}^{\prime} \ldots A_{p+1}^{\prime}\right)}+\nabla_{\left[A_{1}\right.}^{\left(A_{1}^{\prime}\right.} f_{A_{2} \ldots A_{q+1}}^{\left.A_{2}^{\prime} \ldots A_{p+1}^{\prime}\right)}\right. \\
& \left.-\varepsilon^{\left(A_{1}^{\prime} A_{j}^{\prime}\right.} \Upsilon_{A D^{\prime}} f_{\left.A_{1} \ldots A_{q+1}\right]}^{\left.A_{2}^{\prime} \ldots\left|D^{\prime}\right| \ldots A_{p+1}^{\prime}\right)}-\Upsilon_{\left[A_{j}\right.}^{\left(A_{1}^{\prime}\right.} f_{\left.A_{2} \ldots A_{1} \ldots A_{q+1}\right]}^{\left.A_{2}^{\prime} \ldots A_{p+1}^{\prime}\right)}\right\} .
\end{aligned}
$$

Here $\widetilde{\nabla}_{A_{1}}^{A_{1}^{\prime}} \Omega=\widetilde{\nabla}_{A_{1} B^{\prime}} \Omega \cdot \widetilde{\varepsilon}^{B^{\prime} A_{1}^{\prime}}=Z_{A_{1} B^{\prime}} \Omega \cdot \widetilde{\varepsilon}^{B^{\prime} A_{1}^{\prime}}=\Omega^{-1} Z_{A_{1}}^{A_{1}^{\prime}} \Omega=\Upsilon_{A_{1}}^{A_{1}^{\prime}}$, and the third term in the bracket above vanishes automatically. While for the fourth term in the bracket, we have

$$
-\Upsilon_{\left[A_{j}\right.}^{\left(A_{1}^{\prime}\right.} f_{\left.A_{2} \ldots A_{1} \ldots A_{q+1}\right]}^{\left.A_{2}^{\prime} \ldots A_{p+1}^{\prime}\right)}=\Upsilon_{\left[A_{1}\right.}^{\left(A_{1}^{\prime}\right.} f_{\left.A_{2} \ldots A_{j} \ldots A_{q+1}\right]}^{\left.A_{2}^{\prime} \ldots A_{p+1}^{\prime}\right)}, \quad j=2, \ldots, q+1,
$$

and so the first and fourth terms in (A.29) cancel. The first identity in (3.22) follows. Similarly, we have

$$
\begin{aligned}
\left(\widetilde{\mathscr{D}}_{q, p}\left(\Omega^{-q-1} f\right)\right)_{A_{1} \ldots A_{q+1} A_{2}^{\prime} \ldots A_{p}^{\prime}}=-(q+1) \Omega^{-q-2} \Upsilon_{\left[A_{1}\right.}^{A^{\prime}} f_{\left.A_{2} \ldots A_{q+1}\right] A^{\prime} A_{2}^{\prime} \ldots A_{p}^{\prime}}+\Omega^{-q-1} \widetilde{\nabla}_{\left[A_{1}\right.}^{A^{\prime}} f_{\left.A_{2} \ldots A_{q+1}\right] A^{\prime} A_{2}^{\prime} \ldots}, & =\Omega^{-q-2}\{ \\
& -(q+1) \Upsilon_{\left[A_{1}\right.}^{A^{\prime}} f_{\left.A_{2} \ldots A_{q+1}\right] A^{\prime} A_{2}^{\prime} \ldots A_{p}^{\prime}}+\nabla_{\left[A_{1}\right.}^{A^{\prime}} f_{\left.A_{2} \ldots A_{q+1}\right] A^{\prime} A_{2}^{\prime} \ldots A_{p}^{\prime}} \\
& -\Upsilon_{\left[A_{j}\right.}^{A^{\prime}} f_{\left.A_{2} \ldots A_{1} \ldots A_{q+1}\right] A^{\prime} A_{2}^{\prime} \ldots A_{p}^{\prime}}-\Upsilon_{A^{\prime}\left[A_{1}\right.} f_{\left.A_{2} \ldots A_{q+1}\right]} A_{A_{2}^{\prime} \ldots \ldots A_{p}^{\prime}} \\
& \left.-\Upsilon_{A_{j}^{\prime}\left[A_{1}\right.} f_{\left.A_{2} \ldots A_{q+1}\right] A^{\prime} A_{2}^{\prime} \ldots} A^{\prime} \ldots A_{p}^{\prime}\right\} .
\end{aligned}
$$

The last term in the bracket above vanishes by (A.26) and $f$ symmetric in primed indices. We also use (A.26) to raise and lower $A^{\prime}$ in the 4 th term. The result follows from an identity similar to (A.30) that the sum of the third and 4th terms in the bracket cancels the first term. We get the second identity in (3.22). At last

$$
\begin{aligned}
& \widetilde{\nabla}_{A^{\prime}\left[A_{1}\right.} \widetilde{\nabla}_{A_{2}}^{A^{\prime}}\left(\Omega^{-q-1} f\right)_{\left.A_{3} \ldots A_{q+2}\right]} \\
& =\widetilde{\nabla}_{A^{\prime}\left[A_{1}\right.}\left\{\Omega^{-q-2}\left(-(q+1) \Upsilon_{\left[A_{2}\right.}^{A^{\prime}} f_{\left.\left.A_{3} \ldots A_{q+2}\right]\right]}+\nabla_{\left[A_{2}\right.}^{A^{\prime}} f_{\left.\left.A_{3} \ldots A_{q+2}\right]\right]}-\Upsilon_{\left[A_{m}\right.}^{A^{\prime}} f_{\left.\left.A_{3} \ldots A_{2} \ldots A_{q+2}\right]\right]}\right)\right\} \\
& =\widetilde{\nabla}_{A^{\prime}\left[A_{1}\right.}\left\{\Omega^{-q-2}\left(-\Upsilon_{A_{2}}^{A^{\prime}}+\nabla_{A_{2}}^{A^{\prime}}\right) f_{\left.A_{3} \ldots A_{q+2}\right]}\right\} \\
& =\Omega^{-q-2}\left((q+2) \Upsilon_{A^{\prime}\left[A_{1}\right.} \Upsilon_{A_{2}}^{A^{\prime}}-(q+2) \Upsilon_{A^{\prime}\left[A_{1}\right.} \nabla_{A_{2}}^{A^{\prime}}-\nabla_{A^{\prime}\left[A_{1}\right.} \Upsilon_{A_{2}}^{A^{\prime}}-\Upsilon_{\left[A_{2}\right.}^{A^{\prime}} \nabla_{\left|A^{\prime}\right| A_{1}}+\nabla_{A^{\prime}\left[A_{1}\right.} \nabla_{A_{2}}^{A^{\prime}}\right) f_{\left.A_{3} \ldots A_{q+2}\right]} \\
& +\Omega^{-q-2}\left(\Upsilon_{A^{\prime}\left[A_{j}\right.} \Upsilon_{A_{2}}^{A^{\prime}}-\Upsilon_{A^{\prime}\left[A_{j}\right.} \nabla_{A_{2}}^{A^{\prime}}\right) f_{\left.\ldots A_{1} \ldots A_{q+2}\right]}+\delta_{A^{\prime}} A^{A^{\prime}} \Omega^{-q-2}\left(-\Upsilon_{D^{\prime}\left[A_{1}\right.} \Upsilon_{A_{2}}^{D^{\prime}}+\Upsilon_{D^{\prime}\left[A_{1}\right.} \nabla_{A_{2}}^{D^{\prime}}\right) f_{\left.A_{3} \ldots A_{q+2}\right]}
\end{aligned}
$$


where $m=3, \ldots, q+2, j=2, \ldots, q+2$. Here we use the identity as A.30 in the second identity. Note that terms of the form $\Upsilon_{A^{\prime}\left[A_{1}\right.} \nabla_{A_{2}}^{A^{\prime}} f_{\text {...] }}$ cancel each other by $-\Upsilon_{\left[A_{2}\right.}^{A^{\prime}} \nabla_{\left.\left|A^{\prime}\right| A_{1}\right]}=-\Upsilon_{A^{\prime}\left[A_{1}\right.} \nabla_{\left.A_{2}\right]}^{A^{\prime}}$ by using (A.26) to raise and lower primed indices, and only one of terms of the form $-\Upsilon_{A^{\prime}\left[A_{1}\right.} \Upsilon_{A_{2}}^{A^{\prime}} f_{\ldots]}$ remains. So

$$
\begin{aligned}
\widetilde{\nabla}_{A^{\prime}\left[A_{1}\right.} \widetilde{\nabla}_{A_{2}}^{A^{\prime}}\left(\Omega^{-q-1} f\right)_{\left.A_{3} \ldots A_{q+2}\right]} & =\Omega^{-q-2}\left(-\Upsilon_{A^{\prime}\left[A_{1}\right.} \Upsilon_{A_{2}}^{A^{\prime}}-\nabla_{A^{\prime}\left[A_{1}\right.} \Upsilon_{A_{2}}^{A^{\prime}}+\nabla_{A^{\prime}\left[A_{1}\right.} \nabla_{A_{2}}^{A^{\prime}}\right) f_{\left.A_{3} \ldots A_{q+2}\right]} \\
& =\Omega^{-q-2}\left(-2 \Omega \widetilde{\Lambda}_{\left[A_{1} A_{2}\right.}+2 \Lambda_{\left[A_{1} A_{2}\right.}+\nabla_{A^{\prime}\left[A_{1}\right.} \nabla_{A_{2}}^{A^{\prime}}\right) f_{\left.A_{3} \ldots A_{q+2}\right]},
\end{aligned}
$$

by conformal transformation formula for $\Lambda$ in (3.21). We get the last identity in (3.22).

We need the following theorem to prove Theorem 3.2

Theorem A.1. (Theorem 4.12 in [49]) Let $L$ be a self-adjoint and elliptic differential operator of order $m$ on a vector bundle $F$ over a compact manifold. Then there exist linear mappings $H_{L}, G_{L}: C^{\infty}(X, F) \longrightarrow$ $C^{\infty}(X, F)$ so that

(1) $H_{L}\left(C^{\infty}(X, F)\right)=\mathfrak{H}_{L}(E)$ and $\operatorname{dim} \mathfrak{H}_{L}(E)<\infty$.

(2) $L \circ G_{L}+H_{L}=G_{L} \circ L+H_{L}=$ the identity on $C^{\infty}(X, F)$.

(3) $H_{L}$ and $G_{L}$ extend to bounded linear operators on $L^{2}(X, F)$.

(4) $C^{\infty}(X, F)=\mathfrak{H}_{L}(F) \oplus L \circ G_{L}\left(C^{\infty}(X, F)\right)=\mathfrak{H}_{L}(F) \oplus G_{L} \circ L\left(C^{\infty}(X, F)\right)$ is an orthogonal decomposition with respect to the inner product in $L^{2}(X, F)$. In particular, we have $\mathfrak{H}_{L}(F)=\operatorname{ker} L$.

Proof of Theorem [3.2. We only give the proof of case $j=k$. Other cases are similar (see [48] [45] for the flat case).

We will omit the superscript $(k)$ for simplicity. Applying Theorem A.1 to the elliptic differential operators $L=\square_{k}$ of 4 -th order, we see that that there exists a partial inverse operator $\mathbf{G}_{k}: \Gamma\left(\mathscr{V}_{k}\right) \longrightarrow$ $\Gamma\left(\mathscr{V}_{k}\right)$ such that

$$
\mathbf{G}_{k} \square_{k}=\square_{k} \mathbf{G}_{k}=\text { id } \quad \text { on } \quad\left(\operatorname{ker} \square_{k}\right)^{\perp} .
$$

To see ker $\square_{k}=\mathscr{H}_{(k)}^{k}(M)$, note that $\left(\square_{k} f, f\right)=0$ implies that $\left(D_{k} f, D_{k} f\right)=0$ and $\left(D_{k-1} D_{k-1}^{*} f\right.$, $\left.D_{k-1} D_{k-1}^{*} f\right)=0$, i.e. $D_{k} f=0$ and $D_{k-1} D_{k-1}^{*} f=0$. Then we have $D_{k-1}^{*} f=0$ by

$$
\left(D_{k-1} D_{k-1}^{*} f, f\right)=\left(D_{k-1}^{*} f, D_{k-1}^{*} f\right)=0 .
$$

Now suppose that $f \in \Gamma\left(\mathscr{V}_{k}\right)$ such that $f \perp$ (Image $\left.D_{k}^{*} \oplus \mathscr{H}_{(k)}^{k}(M)\right)$. Then obviously $f \in\left(\operatorname{ker} \square_{k}\right)^{\perp}$ since $\operatorname{ker} \square_{k}=\mathscr{H}_{(k)}^{k}(M)$, and $D_{k} f=0$ by $\left(D_{k} f, h\right)=\left(f, D_{k}^{*} h\right)=0$ for any $h \in \Gamma\left(\mathscr{V}_{k+1}\right)$. The decomposition (3.39) holds if we can show $f \in$ Image $D_{k-1}$. If set

$$
u:=D_{k-1}^{*} D_{k-1} D_{k-1}^{*} \mathbf{G}_{k} f \in \Gamma\left(\mathscr{V}_{k-1}\right),
$$

we have

$$
D_{k-1} u=\left(D_{k-1} D_{k-1}^{*}\right)^{2} \mathbf{G}_{k} f=\left(\left(D_{k-1} D_{k-1}^{*}\right)^{2}+D_{k}^{*} D_{k}\right) \mathbf{G}_{k} f=\square_{k} \mathbf{G}_{k} f=f,
$$

by (A.31) in the last identity and

$$
D_{k}^{*} D_{k} \mathbf{G}_{k} f=0 .
$$

This is because

$$
\left(D_{k}^{*} D_{k} \mathbf{G}_{k} f, D_{k}^{*} D_{k} \mathbf{G}_{k} f\right)=\left(D_{k} \mathbf{G}_{k} f, D_{k} D_{k}^{*} D_{k} \mathbf{G}_{k} f\right)=\left(D_{k} \mathbf{G}_{k} f, \square_{k+1} D_{k} \mathbf{G}_{k} f\right)=0
$$

by

$$
D_{k}\left(D_{k}^{*} D_{k}+\left(D_{k-1} D_{k-1}^{*}\right)^{2}\right)=D_{k} D_{k}^{*} D_{k}=\left(\left(D_{k+1}^{*} D_{k+1}\right)^{2}+D_{k} D_{k}^{*}\right) D_{k} .
$$


and so

$$
\square_{k+1} D_{k} \mathbf{G}_{k} f=D_{k} \square_{k} \mathbf{G}_{k} f=D_{k} f=0 .
$$

The commutating relation A.33 follows from $D_{k} D_{k-1}=D_{k+1} D_{k}=0$. The result follows.

\section{REFERENCES}

[1] Adams, W., Loustaunau, P., Palamodov, V. and Struppa, D., Hartogs' phenomenon for polyregular functions and projective dimension of releted modules over a polynomial ring, Ann. Inst. Fourier 47 (1997) 623-640.

[2] Alesker, S., Pluripotential theory on quaternionic manifolds, J. Geom. Phys. 62 (2012), no. 5, 1189-1206.

[3] Alesker, S. and Verbitsky, M. , Quaternionic Monge-Ampère equation and Calabi problem for HKT-manifolds, Israel J. Math. 176 (2010), 109-138.

[4] Alesker, S. and Verbitsky, M., Plurisubharmonic functions on hypercomplex manifolds and HKT-geometry, $J$. Geom. Anal. 16 (2006) 375-399.

[5] Bailey, T. And Eastwood, M., Complex paraconformal manifolds-their differential geometry and twistor theory, Forum Math. 3 (1991), 61-103.

[6] Baston, R., Quaternionic complexes, J. Geom. Phys. 8 (1992) 29-52.

[7] Baston, R. and Eastwood, M., The Penrose transform. Its interaction with representation theory, Oxford Mathematical Monographs, The Clarendon Press, Oxford University Press, New York, 1989.

[8] Bergshoeff, E., de Wit, T., Halbersma, R., Cucu, S., Gheerardyn, J., Van Proeyen, A. and Vandoren, S., Superconformal $N=2, D=5$ matter with and without actions, J. High Energy Phys. 2002, no. 10, 045, 65 pp.

[9] Burěs, J. ANd V. SouČEK, V., Complexes of invariant differential operators in several quaternionic variables, Complex Var. Elliptic Equ. 51 (2006), no. 5-6, 463-487.

[10] Bureš, J., Damiano, A. and Sabadini, I., Explicit resolutions for several Fueter operators, J. Geom. Phys. 57, 2007, 765-775.

[11] ČAP, A. And Slovak, J., Parabolic geometries I. Background and general theory, Mathematical Surveys and Monographs 154, American Mathematical Society, Providence, RI, 2009.

[12] ČAP, A., Slovak, J. And SouČEK, V., Invariant operators on manifolds with almost Hermitian symmetric structures. III. Standard operators, Differential Geom. Appl. 12 (2000), no. 1, 51-84.

[13] ČAp, A., SlovaK, J. And SouČEK, V., Bernstein-Gelfand-Gelfand sequences, Ann. of Math. (2) 154 (2001), 97-113.

[14] Cap, A. And Souček, V., Subcomplexes in curved BGG-sequences, Math. Ann. 354 (2012), no. 1, 111-136.

[15] Chang, D.-C., Markina, I. And Wang, W., On the Hodge-type decomposition and cohomology groups of $k$-CauchyFueter complexes over domains in the quaternionic space, J. Geom. Phys. 107 (2016), 15-34.

[16] Cherney, D., Latini, E. and Waldron, A., Quaternionic Kähler detour complexes and $N=2$ supersymmetric black holes, Comm. Math. Phys. 302 (2011), no. 3, 843-873.

[17] Colombo, F., Souček, V. and Struppa, D., Invariant resolutions for several Fueter operators, J. Geom. Phys. 56 (2006), no. 7, 1175-1191.

[18] Colombo, F., Sabadini, I., Sommen, F. And Struppa, D., Analysis of Dirac systems and computational algebra, Progress in Mathematical Physics 39, Boston, Birkhäuser, 2004.

[19] Eastwood, M., Penrose, R. and Wells, R., Cohomology and massless fields, Comm. Math. Phys.. 78 (1980), no. $3,305-351$.

[20] Hitchin, N.J., Karlhede, A., Lindström, U. And RočEK, M., HyperKähler metrics and supersymmetry, Commun. Math. Phys. 108 (1987), 535-589.

[21] Homma, Y., Estimating the eigenvalues on Quaternionic Kahler Manifolds, International Jouranal of Mathematics 17(6) (2006), 665-691.

[22] Horan, R., A rigidity theorem for quaternionic Kähler manifolds, Diff. Geom. and Appl. 6 (1996) 189-196.

[23] Horan, R., Cohomology of a quaternionic complex, in Further advances in twistor theory III. curved twistor spaces, edit by L. Mason et al., Chapman and Hall/CRC, London, New York, (2000), 66-71.

[24] Ivanov, S. And Minchev, I., Quaternionic Kähler and hyperKähler manifolds with torsion and twistor spaces, J. Reine Angew. Math. 567 (2004), 215-233.

[25] Kang, Q. And Wang, W., On Penrose integral formula and series expansion of $k$-regular functions on the quaternionic space $\mathbb{H}^{n}$, J. Geom. Phys. 64 (2013), 192-208.

[26] Nagatomo, Y. and Nitta, T., Vanishing theorems for quaternionic complexes, Bull. London Math. Soc. 29 (1997), no. 3, 359-366. 
[27] Neitzke, A., Pioline, B. And Vandoren, S., Twistors and black holes, JHEP 0704, 038 (2007).

[28] Ornea, L. And Piccini, P., Locally conformally Kähler structures in quaternionic geometry, Trans. Am. Math. Soc. 349 (1997) 641-655.

[29] Pandžı́, P. And V. SoučEk, V., BGG complexes in singular infinitesimal character for type A, J. Math. Phys. 58 (2017), 111512.

[30] Penrose, R. And Rindler, W., Spinors and Space-Time, Vol. 1, Two-spinor calculus and relativistic fields, Cambridge Monographs on Mathematical Physics, Cambridge University Press, Cambridge, 1984.

[31] Penrose, R. and Rindler, W., Spinors and Space-Time, Vol. 2, Spinor and twistor methods in space-time geometry, Cambridge Monographs on Mathematical Physics, Cambridge University Press, Cambridge, 1986.

[32] Salamon, S., Quaternionic Kähler manifolds, Invent. Math. 67, (1982), no. 1, 143-171.

[33] Salamon, S., Differential geometry of quaternionic manifolds, Ann. Sci. École Norm. Sup. (4) 19 (1986), no. 1, 31-55.

[34] Semmelmann, U. And Weingart, G., Vanishing theorems for quaternionic Kähler manifolds, J. Reine Angew. Math. 544 (2002), 111-132.

[35] Semmelmann, U. and Weingart, G., The Weitzenböck machine, Compos. Math. 146 (2010), no. 2, 507-540.

[36] Slovak, J. And Souček, V., Invariant operators of the first order on manifolds with a given parabolic structure, in Global analysis and harmonic analysis (Marseille-Luminy, 1999), 251-276, Semin. Congr. 4, Soc. Math. France, Paris, 2000.

[37] Spacil, O., Indices of quaternionic complexes, Diff. Geom. and Appl. 28 (2010), 395-405.

[38] Swann, A., Hyper-Kähler and quaternionic Kähler geometry, Math. Ann. 289 (1991), no. 3, 421-450.

[39] Verbitsky, M., Quaternionic Dolbeault complex and vanishing theorems on hyperKähler manifolds, Compos. Math. 143 (2007), no. 6, 1576-1592.

[40] Wan, D. , The continuity and range of the quaternionic Monge-Ampère operator on quaternionic space, Math. Zeit. 285 (2017), 461-478.

[41] Wan, D. And Kang, Q., Potential theory for quaternionic plurisubharmonic functions, Michigan Math. J. 66 (2017), no. $1,3-20$.

[42] Wan, D. and Zhang, W. J., Quasicontinuity and maximality of quaternionic plurisubharmonic functions, J. Math. Anal. Appl. 424 (2015), no. 1, 86-103.

[43] WAN, D. AND WANG, W., On the quaternionic Monge-Ampère operator, closed positive currents and Lelong-Jensen type formula on quaternionic space, Bull. Sci. Math. 141 (2017), no. 4, 267-311.

[44] Wang, H. Y. and Ren, G. B., Bochner-Martinelli formula for k-Cauchy-Fueter operator, J. Geom. Phys. 84 (2014), 43-54.

[45] Wang, W., The $k$-Cauchy-Fueter complexes, Penrose transformation and Hartogs' phenomenon for quaternionic $k$ regular functions, J. Geom. Phys. 60, (2010), 513-530.

[46] Wang, W., On twistor transformations and invariant differential operator of simple Lie group $G_{2(2)}$, J. Math. Phys. 54 (2013), 013502.

[47] WANG, W., On the weighted $L^{2}$ estimate for the $k$-Cauchy-Fueter operator and the weighted $k$-Bergman kernel, $J$. Math. Anal. Appl. 452 (2017), no. 1, 685-707.

[48] Wang, W., The Neumann problem for the $k$-Cauchy-Fueter complexes over $k$-pseudoconvex domains in $\mathbb{R}^{4}$ and the $L^{2}$ estimate, to appear in J. Geom. Anal., arXiv:1704.02856

[49] Wells, R., Differential analysis on complex manifolds, Graduate Texts in Mathematics 65, Springer-Verlag, New York-Berlin, 1980. 